\title{
Thermonuclear Stability of Material Accreting onto a Neutron Star
}

\section{Citation}

Narayan, Ramesh, and Jeremy S. Heyl. 2003. “Thermonuclear Stability of Material Accreting onto a Neutron Star." The Astrophysical Journal 599 (1): 419-49. https://doi.org/10.1086/379211.

\section{Permanent link}

http://nrs.harvard.edu/urn-3:HUL.InstRepos:41384951

\section{Terms of Use}

This article was downloaded from Harvard University's DASH repository, and is made available under the terms and conditions applicable to Other Posted Material, as set forth at http:// nrs.harvard.edu/urn-3:HUL.InstRepos:dash.current.terms-of-use\#LAA

\section{Share Your Story}

The Harvard community has made this article openly available.

Please share how this access benefits you. Submit a story.

\section{Accessibility}




\title{
Thermonuclear Stability of Material Accreting onto a Neutron Star
}

\author{
Ramesh Narayan ${ }^{1}$ and Jeremy S. Heyl ${ }^{1,2,3}$ \\ rnarayan@cfa.harvard.edu; jheyl@cfa.harvard.edu
}

\begin{abstract}
We present a global linear stability analysis of nuclear fuel accumulating on the surface of an accreting neutron star and we identify the conditions under which thermonuclear bursts are triggered. The analysis reproduces all the recognized regimes of hydrogen and helium bursts, and in addition shows that at high accretion rates, near the limit of stable burning, there is a regime of "delayed mixed bursts" which is distinct from the more usual "prompt mixed bursts." In delayed mixed bursts, a large fraction of the fuel is burned stably before the burst is triggered. Bursts thus have longer recurrence times, but at the same time have somewhat smaller fluences. Therefore, the parameter $\alpha$, which measures the ratio of the energy released via accretion to that generated through nuclear reactions in the burst, is up to an order of magnitude larger than for prompt bursts. This increase in $\alpha$ near the threshold of stable burning has been seen in observations. We explore a wide range of mass accretion rates, neutron star radii and core temperatures, and calculate a variety of burst properties. From a preliminary comparison with data, we suggest that bursting neutron stars may have hot cores, with $T_{\text {core }} \gtrsim 10^{7.5} \mathrm{~K}$, consistent with interior cooling via the modified URCA or similar low-efficiency process, rather than $T_{\text {core }} \sim 10^{7} \mathrm{~K}$, as expected for the direct URCA process. There is also an indication that neutron star radii are somewhat small $\lesssim 10 \mathrm{~km}$. Both of these conclusions need to be confirmed by comparing more careful calculations with better data.
\end{abstract}

Subject headings: accretion - X-rays: binaries, bursts

\footnotetext{
${ }^{1}$ Harvard-Smithsonian Center for Astrophysics, Cambridge, MA 02138, U.S.A.

${ }^{2}$ Chandra Fellow

${ }^{3}$ Current Address: Department of Physics and Astronomy, University of British Columbia, Vancouver, BC V6T 1Z1, Canada
} 


\section{Introduction}

When gas accretes onto a neutron star, nuclear reactions often occur in an unstable fashion (Hansen \& van Horn 1975), leading to thermonuclear explosions which are called Type I X-ray bursts. These bursts were first observed by Grindlay et al. (1976), and have been studied intensively for many years (see van Paradijs et al. 1988; Lewin et al. 1993; Strohmayer et al. 1998; Cornelisse et al. 2003, for summaries of the observations). Recently, there has been renewed excitement in the field, following the discovery of high frequency oscillations in burst light curves (e.g., Strohmayer et al. 1996; Strohmayer 2001a; van Straaten et al. 2001; Muno et al. 2001).

The physics of Type I bursts has been widely studied, and the broad features of the phenomenon are understood theoretically (Woosley \& Taam 1976; Joss 1977; Taam \& Picklum 1978; Paczynski 1983a; Fujimoto et al. 1981; Fushiki \& Lamb 1987a; Taam et al. 1996; Bildsten 1998). Models show that there are three kinds of bursts, depending on whether hydrogen or helium burning dominates. At high mass accretion rates $\dot{M}$, hydrogen is only partially burned before a burst is triggered by unstable helium burning. The result is a mixed burst in which both hydrogen and helium burn explosively. At somewhat lower values of $\dot{M}$, all the hydrogen is consumed before the helium instability is triggered. This leads to a pure helium burst. For yet lower $\dot{M}$, hydrogen itself burns unstably, giving a hydrogen burst. The ranges of $\dot{M}$ corresponding to the different regimes have been worked out approximately.

Two very different approaches have been pursued for theoretically modeling the burst phenomenon. In one approach, one simulates the physics of the accreting gas with a fully time-dependent code that includes a large network of nuclear reactions and sophisticated thermodynamics (e.g., Joss 1978; Taam \& Picklum 1979; Joss \& Li 1980; Taam 1982; Fu-

jimoto et al. 1987b; Taam et al. 1993). Such studies are essential for understanding the details of the thermonuclear explosion; indeed, the one-dimensional simulations of the past have now been generalized to two-dimensional and even three-dimensional simulations (the FLASH effort at Chicago, Zingale et al. 2001) which follow the physics of bursts in exquisite detail. Such simulations are, however, not very convenient for parameter surveys or for detailed comparisons of theoretical predictions with observational burst statistics.

An alternate approach, where one focuses on the thermonuclear instability that triggers the burst rather than on the burst itself, has been popular (e.g., Hansen \& van Horn 1975; Ergma \& Tutukov 1980; Fujimoto et al. 1981; Taam 1982; Paczynski 1983a; Fushiki \& Lamb 1987a; Fujimoto et al. 1987a; Cumming \& Bildsten 2000). Here one treats the accumulating layer of gas on the surface of the neutron star as a quasi-equilibrium system whose properties vary slowly with time. One first solves for the equilibrium structure and then analyses the stability of the gas layer by considering the effect of small perturbations on the underlying 
equilibrium. If the perturbations grow with time, one says that the system is unstable, presumably resulting in a Type 1 X-ray burst.

The second approach, though not as general as the first, allows one to explore a large range of parameter space and to study how the burst phenomenon is affected by variations in control parameters such as the mass accretion rate, the surface gravity of the star, etc. However, the analyses that have been published so far in the literature employ fairly simpleminded criteria to decide exactly when an accreted layer becomes unstable and are, therefore, not very accurate or complete. The motivation of the present paper is to develop a more rigorous stability analysis for accreting neutron stars. Our hope is (i) to verify the results of the earlier methods, (ii) to explore additional phenomena that may have been missed previously, and (iii) to put the theory on a rigorous footing to enable quantitative comparisons with observations. We reported some early results of this work in Narayan \& Heyl (2002).

We begin the paper in $\S 2$ with a description of our model of the accreted layer and the method we employ to calculate equilibria and to study their stability. We follow this in $\S 3$ with an exploration of sequences of equilibria, and in $\S 4$ with a discussion of the stability properties of the equilibria. Through this analysis, we reproduce the three previously recognized regimes of burst activity, namely mixed bursts, helium bursts, and hydrogen bursts. However, we find that mixed bursts themselves come in two kinds: prompt mixed bursts and delayed mixed bursts. The latter category has not been recognized previously. In $\S 5$ we present results for neutron stars of various radii and core temperatures, and explore a wide range of mass accretion rates. We calculate a number of burst observables such as the recurrence time, the burst duration, and the dimensionless parameter $\alpha$ (Eq. 35) which is widely used in the burst literature. In $\S 6$, we compare our theoretical formalism to methods previously published in the literature. We also compare our predictions with selected observations. From the latter, we obtain preliminary results on the likely core temperatures and radii of bursting neutron stars. We conclude in $\S 7$ with a summary.

\section{The Model}

\subsection{Governing Equations}

We assume that gas accretes on the surface of a compact spherical star of mass $M$

and radius $R$ at a rate $\dot{\Sigma}$ (mass per unit area per unit time). We consider all quantities to be functions of $\Sigma$, the column density (mass per unit area) measured from the top of the accreted layer. We use partial derivatives $\partial / \partial t$ and $\partial / \partial \Sigma$ to signify Eulerian time and "spatial" derivatives at a fixed $\Sigma$, and $d / d t$ to represent the "Lagrangian" time derivative 
following a parcel of accreted gas (see Fushiki \& Lamb 1987a):

$$
\frac{d}{d t} \equiv \frac{\partial}{\partial t}+\dot{\Sigma} \frac{\partial}{\partial \Sigma}
$$

We consider only hydrogen and helium burning, and so we describe the composition of the gas in terms of the hydrogen mass-fraction $X$, the helium mass fraction $Y$, and the heavy element fraction $Z=1-X-Y$. These quantities start off with values $X_{\text {out }}, Y_{\text {out }}, Z_{\text {out }}$ at $\Sigma=0$, corresponding to the composition of the gas initially falling on the neutron star, and evolve with increasing depth as a result of nuclear burning. Because $H$-burning is mostly done via the CNO-cycle, it is necessary to know what fraction of $Z$ is in CNO elements (see Eq. 26). For this, we assume that about $80 \%$ of the initial $Z_{\text {out }}$ is in CNO, as appropriate for solar composition (Allen 2000), and that all the $Z$ produced via helium burning is entirely in $\mathrm{CNO}$; thus, we take

$$
Z_{\mathrm{CNO}}=0.8 Z_{\text {out }}+\left(Z-Z_{\text {out }}\right)
$$

The time evolution of the accreting gas is described by a set of five coupled partial differential equations:

$$
\begin{aligned}
\frac{\partial P}{\partial \Sigma} & =g, \\
\frac{\partial T}{\partial \Sigma} & =\frac{3 \kappa F}{16 \sigma T^{3}}, \\
\frac{\partial F}{\partial \Sigma} & =-T \frac{\mathrm{d} s}{\mathrm{~d} t}-\left(\epsilon_{\mathrm{H}}+\epsilon_{\mathrm{He}}\right), \\
\frac{\mathrm{d} X}{\mathrm{~d} t} & =-\frac{\epsilon_{\mathrm{H}}}{E_{\mathrm{H}}^{*}}, \\
\frac{\mathrm{d} Y}{\mathrm{~d} t} & =\frac{\epsilon_{\mathrm{H}}}{E_{\mathrm{H}}^{*}}-\frac{\epsilon_{\mathrm{He}}}{E_{\mathrm{He}}^{*}} .
\end{aligned}
$$

Table 1 gives the definitions of the various symbols. We assume that the accreted layer is thin relative to the stellar radius, and so we take the gravitational acceleration $g$ to be independent of $\Sigma$ :

$$
g=(1+z) \frac{G M}{R^{2}}, \quad 1+z=\left(1-\frac{2 G M}{c^{2} R}\right)^{-1 / 2}
$$

where $z$ is the gravitational redshift. Note that $g$ and all other quantities in equations $(3-7)$ are measured in the local frame of the gas. In this spirit, $\dot{\Sigma}$ is the baryonic mass added per unit surface area per unit local time.

Equation (3) describes the condition of hydrostatic equilibrium. By making use of this equation rather than the full momentum equation, we filter out sound waves and focus on 
Table 1. Definition of symbols:

\begin{tabular}{|c|c|}
\hline Symbol & Meaning \\
\hline$R, M, R_{S}$ & stellar radius, stellar mass, Schwarzschild radius: $2 G M / c^{2}$ \\
\hline$g z$ & gravitational acceleration, redshift at the surface \\
\hline & mass accretion rate per unit area \\
\hline$L_{\mathrm{acc}}, L_{\mathrm{Edd}}, l_{\mathrm{acc}}$ & accretion luminosity, Eddington luminosity, $l_{\mathrm{acc}}=L_{\mathrm{acc}} / L_{\mathrm{Edd}}$ \\
\hline & surface mass density measured from the surface; independent variable in eqs \\
\hline$\Sigma_{\text {layer }}$ & $\Sigma$ of the accreted layer \\
\hline$\Sigma_{\text {layer,crit }}$ & critical $\Sigma_{\text {layer }}$ at which a burst is triggered \\
\hline$\Sigma_{\text {diff }}, \Sigma_{\max }$ & Maximum integration depth for the equilibrium, and for perturbations \\
\hline$t, P, \rho, T, s$ & time, pressure, mass density, temperature, entropy per unit mass \\
\hline$F$ & outbound energy flux \\
\hline$X, Y, Z$ & mass fractions of hydrogen, helium, metals \\
\hline$Z_{\mathrm{CNO}}$ & mass fraction of CNO nuclei \\
\hline$\rho_{0}, T_{0} ; \rho_{1}, T_{1}$ & values in equilibrium; perturbations \\
\hline$T_{\text {out }}, X_{\text {out }}$, etc. & values at the surface \\
\hline$F_{\text {nuc }}$ & expected surface flux if entire fuel is steadily burned \\
\hline$F_{\text {out }}, f_{\text {nuc }}$ & escaping flux due to nuclear reactions and compression, $f_{\text {out }}=F_{\text {out }} / F_{\text {nuc }}$ \\
\hline$F_{\text {acc }}$ & persistent flux from the surface due to accretion: $\dot{\Sigma} c^{2} z /(1+z)$ \\
\hline$T_{\text {layer }}, X_{\text {layer }}$, etc. & values at the bottom of the accreted layer \\
\hline$T_{\max }, T_{\text {core }}$ & maximum $T$ in layer, core temperature \\
\hline$\tau_{\text {diff }}$ & thermal diffusion time \\
\hline$\kappa, K$ & opacity, conductivity \\
\hline$\epsilon_{\mathrm{H}, \mathrm{He}}$ & energy-generation rate per unit mass for $\mathrm{H}$, He burning \\
\hline$E_{\mathrm{H}, \mathrm{He}}^{*}$ & total nuclear energy released per unit mass of $\mathrm{H}, \mathrm{He}$ burned \\
\hline$\gamma$ & growth rate of mode, $\gamma=\Re(\gamma)+i \Im(\gamma)$ \\
\hline$\gamma_{\mathrm{acc}}, t_{\mathrm{acc}}$ & $\gamma_{\text {acc }}=\dot{\Sigma} / \Sigma_{\text {layer }}$, accretion rate; $t_{\text {acc }}=\Sigma_{\text {layer }} / \dot{\Sigma}$, accretion time \\
\hline & burst recurrence time: $(1+z) \Sigma_{\text {layer,crit }} / \dot{\Sigma}$ \\
\hline$E_{\mathrm{H}}, E_{\mathrm{He}}$ & fluence in burst from burning hydrogen, helium \\
\hline$t_{\mathrm{H}+\mathrm{He}}, t_{\mathrm{He}}$ & effective burst duration: $\left(E_{\mathrm{H}}+E_{\mathrm{He}}\right) / L_{\mathrm{Edd}}, E_{\mathrm{He}} / L_{\mathrm{Edd}}$ \\
\hline$\alpha$ & ratio of accretion energy to nuclear energy: $\left(t_{\mathrm{rec}} / t_{\mathrm{H}+\mathrm{He}}\right) l_{\mathrm{acc}}$ \\
\hline
\end{tabular}


variations that occur on a much longer time scale than the sound-crossing time. Equation (4) describes energy transfer by radiation and conduction. Equation (5) is the energy conservation equation, and equations (6) and (7) describe the evolution of the hydrogen and helium mass-fractions as a result of nuclear burning. Equations (3)-(7) require expressions for the pressure $P$, the opacity $\kappa$, the entropy $s$, and the energy-generation rates for hydrogen and helium-burning, $\epsilon_{\mathrm{H}}, \epsilon_{\mathrm{He}}$. These are discussed in $\S 2.3$.

The calculations proceed as follows. We start with a bare neutron star and follow the properties of the accreted layer as gas piles up. For a given column density $\Sigma_{\text {layer }}$ of the accreted layer, we calculate the following. First, we solve for the quasi-equilibrium state of the system. To do this, we set the Eulerian time derivative $\partial / \partial t$ to zero in equations (3-7) and consider the following set of ordinary differential equations (see Fushiki \& Lamb 1987a)

$$
\begin{aligned}
\frac{\mathrm{d} P}{\mathrm{~d} \Sigma} & =g \\
\frac{\mathrm{d} T}{\mathrm{~d} \Sigma} & =\frac{3 \kappa F}{16 \sigma T^{3}}, \\
\frac{\mathrm{d} F}{\mathrm{~d} \Sigma} & =-\dot{\Sigma} T \frac{\mathrm{d} s}{\mathrm{~d} \Sigma}-\left(\epsilon_{\mathrm{H}}+\epsilon_{\mathrm{He}}\right), \\
\dot{\Sigma} \frac{\mathrm{d} X}{\mathrm{~d} \Sigma} & =-\frac{\epsilon_{\mathrm{H}}}{E_{\mathrm{H}}^{*}}, \\
\dot{\Sigma} \frac{\mathrm{d} Y}{\mathrm{~d} \Sigma} & =\frac{\epsilon_{\mathrm{H}}}{E_{\mathrm{H}}^{*}}-\frac{\epsilon_{\mathrm{He}}}{E_{\mathrm{He}}^{*}} .
\end{aligned}
$$

We solve these equations with boundary conditions (described in §2.2) to obtain the run of density $\rho_{0}(\Sigma)$, temperature $T_{0}(\Sigma)$, etc., in quasi-steady state. Note that, because $\Sigma_{\text {layer }}$ increases steadily with time, the layer at any given time is not strictly in equilibrium, and the above steady state equations are not precisely valid. However, since $\Sigma_{\text {layer }}$ increases only slowly with time (on the accretion time scale defined in eq. 17 below), and since many of the physical processes in the layer have shorter characteristic time scales, this is a reasonable approximation.

Having calculated the steady state equilibrium solution, we carry out a linear perturbation analysis. This is the principal contribution of our work, and represents a significant advance over previous studies. For the perturbation analysis, we assume that the various physical quantities are functions of $\Sigma$ and $t$ in the form

$$
\begin{aligned}
\rho(\Sigma, t) & =\rho_{0}(\Sigma)+\exp (\gamma t) \rho_{1}(\Sigma) \\
T(\Sigma, t) & =T_{0}(\Sigma)+\exp (\gamma t) T_{1}(\Sigma), \text { etc. }
\end{aligned}
$$

where $\rho_{0}, T_{0}$ represent the solutions obtained from solving the steady state equations described above, and $\rho_{1} \ll \rho_{0}, T_{1} \ll T_{0}$, are small linear perturbations. The frequency $\gamma$ 
represents the growth rate of the perturbations. We substitute the perturbed solution into the original time-dependent equations $(3-7)$ and linearize in the usual way to obtain a set of ordinary differential equations (in $\Sigma$ ) for the first-order quantities $\rho_{1}, T_{1}$, etc. These equations are written down in Appendix A and some of their properties are discussed there. We solve the linearized perturbation equations with appropriate boundary conditions and thereby obtain $\gamma$, which plays the role of an eigenvalue.

In general, there are many solutions for $\gamma$ for a given steady state solution; some values of $\gamma$ are real and some are complex. If any solution for $\gamma$ has a real part sufficiently large compared to the accretion rate - see equation (21) below for a precise statement of what the criterion is - then we say that the layer is unstable. In this case, we identify $\Re(\gamma)$ with the growth rate of the instability in the system. If no solution for $\gamma$ satisfies equation (21), then we consider the system to be stable. Because $\gamma$ is in general complex, all the quantities in the perturbation equations are complex and must be handled with complex arithmetic (in contrast to the steady state equations which involve purely real quantities).

We carry out the above two stages of calculations, namely steady state and linear perturbation analysis, for each $\Sigma_{\text {layer }}$ as matter accumulates on the star. If no instability is found for any choice of $\Sigma_{\text {layer }}$ up to a very large value, typically $10^{13}-10^{14} \mathrm{~g} \mathrm{~cm}^{-3}$ - see Taam \& Picklum (1978) and Brown \& Bildsten (1998) for a discussion of carbon flashes which occur at yet larger column densities - then we say that the system is stable to bursts. If, for

some value of $\Sigma_{\text {layer }}$, we do obtain an instability, then we say that the system will undergo a burst when it accumulates this much gas on its surface.

\subsection{Boundary Conditions}

The solution of the five differential equations (9-13) for the steady state requires five boundary conditions. Four are applied on the outside, at the photosphere (where the optical depth is taken to be $2 / 3$ ), and one is applied below the accreted layer, deep inside the star.

The outer boundary conditions are very similar to the ones employed in Narayan \& Heyl (2002). The surface values of $X$ and $Y$ are set equal to the corresponding values of the accreting gas, namely $X=X_{\text {out }}, Y=Y_{\text {out }}$. We have used a solar composition, $X_{\text {out }}=0.7$, $Y_{\text {out }}=0.28$ (Allen 2000, Table 3.1), in all the calculations reported in this paper. Next, a particular value is assumed for the escaping flux $F_{\text {out }}$ from the accreted layer. This flux is the result of nuclear burning and compression, and its precise value is determined only after applying the inner boundary condition, as explained below. The surface temperature $T_{\text {out }}$ is 
then obtained by the condition

$$
\sigma T_{\text {out }}^{4}=F_{\text {out }}+F_{\text {acc }}, \quad F_{\text {acc }}=\dot{\Sigma} c^{2} z(1+z),
$$

where $F_{\text {acc }}$ is the gravitational energy released by the accreting gas, and we have included the appropriate gravitational redshift factor so that all quantities are calculated in the local frame. The above relation for $T_{\text {out }}$ is approximate, but we have confirmed that the results are very insensitive to the precise choice of $T_{\text {out }}$. The fourth boundary condition is obtained from the radiative transfer equation. Given an assumed value for $F_{\text {out }}$ and the condition $P=0$ at $\Sigma=0$, this equation directly gives the density profile $\rho(\Sigma)$.

As described above, the solution at the surface is completely specified once a value of $F_{\text {out }}$ is assumed. The unknown value of $F_{\text {out }}$ is determined by requiring the solution to satisfy an inner boundary condition. For this, we assume that the accreting star has a specified core temperature $T_{\text {core }}$ and we require the solution of the steady state equations to match this temperature. The key issue is where exactly to do the matching. Our approach is as as follows. Associated with a given accreted column of depth $\Sigma_{\text {layer }}$, there is a characteristic accretion time

$$
t_{\mathrm{acc}}=\frac{\Sigma_{\text {layer }}}{\dot{\Sigma}}
$$

We integrate the steady state equations from the surface down to the bottom of the accreted layer at $\Sigma_{\text {layer }}$, and then we integrate further into the stellar substrate to a depth $\Sigma_{\text {diff }}$ such that the diffusion time from $\Sigma_{\text {layer }}$ to $\Sigma_{\text {diff }}$ is equal to twice $t_{\text {acc }}$ (the factor of 2 is arbitrary and was selected after some numerical experiments):

$$
\tau_{\text {diff }}\left(\Sigma_{\text {diff }}\right)=2 t_{\text {acc }}
$$

For applying this condition, we need an estimate of the thermal diffusion time $\tau_{\text {diff }}(\Sigma)$ for any choice of $\Sigma$ inside the star. We obtain this by integrating a separate differential equation:

$$
\frac{\mathrm{d} \tau_{\text {diff }}}{\mathrm{d} \Sigma}=\frac{9 \kappa k_{B} T\left(\Sigma-\Sigma_{\text {layer }}\right)}{128 \sigma T^{4} \mu m_{u}}
$$

The form of this equation and the numerical coefficient (which is based on a simple toy model) are obtained by treating the energy equation (11) as a diffusion problem. The precise details are unimportant for the final results.

For the calculations presented in this paper, we pick "reasonable" values for $T_{\text {core }}$, trying a range that is likely to bracket the true value. In $\S 5.5$ we will estimate the core temperature as a function of accretion rate for two kinds of neutrino cooling in the core. If we include both the inward directed flux at the bottom of the layer that we calculate and the energy from nuclear reactions in the deep crust (Brown et al. 1998), we find that the latter contribution 
dominates. This yields a simple relationship between the accretion luminosity and the core temperature.

The above treatment of the inner boundary condition is superior to the method used in Narayan \& Heyl (2002, and most other previous studies, see §6.1), where the temperature at the bottom of the accreted layer was set equal to $T_{\text {core }}$. That is, in that calculation, the substrate was assumed to be isothermal immediately below the accreted layer. By integrating down to a couple of diffusion lengths into the substrate, we believe our present approach is physically better motivated. However, even with this approach, we are effectively assuming that the core below $\Sigma_{\text {diff }}$ is perfectly isothermal - hence the use of the term "core temperature" - which is again an approximation. For a system that has been accreting and bursting for a long time, there is expected to be a small time-averaged flux flowing into the

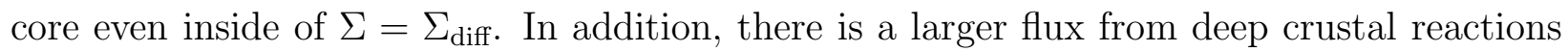
(Brown et al. 1998). Both of these fluxes will induce a temperature gradient, so that the temperature in the crust, which is relevant for bursts, will not be equal to the temperature $T_{\text {core }}$ deep inside the neutron star (set for instance by neutrino cooling, see Brown 2000 for a detailed analysis). This effect may not be very serious, since the matter inside $\Sigma_{\text {diff }}$ is highly degenerate and very conductive. Nevertheless, we mention the point here because the effect of the approximation is presently not fully understood. We believe that the results we present in this paper for $T_{\text {core }} \sim 10^{8} \mathrm{~K}$ will be hardly affected because the burning layer itself has a temperature of this order. However, for very cold cores, e.g., $T_{\text {core }} \sim 10^{7} \mathrm{~K}$, the approximation may have a more serious effect.

The above discussion pertains to the equilibrium solution. The linear perturbation equations have similar boundary conditions. At the surface, the first-order perturbations $X_{1}$ and $Y_{1}$ vanish, and we assume an arbitrary value for the flux perturbation $F_{1}$; the latter choice serves as the overall normalization of the perturbed eigenfunction. From the perturbed flux, the corresponding temperature perturbation $T_{1}$ is readily obtained via equation (16), and finally the density perturbation $\rho_{1}$ is calculated from the radiative transfer equation.

At the bottom, we set the temperature perturbation $T_{1}\left(\Sigma_{\max }\right)$ at a prescribed depth $\Sigma_{\max }$ equal to zero. In analogy with what we did for the steady state solution, we determine $\Sigma_{\max }$ by the condition that the diffusion time down to this depth should be equal to twice the mode time scale $t_{\text {mode }}$ :

$$
\tau_{\text {diff }}\left(\Sigma_{\text {max }}\right)=2 t_{\text {mode }}=\frac{2}{|\gamma|}
$$

where $|\gamma|$ is the modulus of the complex eigenvalue $\gamma$. We only consider modes that grow faster than the accretion time, see below; therefore, $\Sigma_{\max }$ is always smaller than $\Sigma_{\text {diff. The }}$ condition $T_{1}\left(\Sigma_{\max }\right)=0$ provides the final boundary condition that enables us to solve for the eigenmode and the eigenvalue $\gamma$. 
As already mentioned, there are many solutions for $\gamma$. We concentrate on modes that grow fast enough to be interesting, specifically modes that satisfy

$$
\Re(\gamma) \geq g_{\text {mode }} \gamma_{\text {acc }}, \quad g_{\text {mode }}=3, \quad \gamma_{\text {acc }} \equiv \frac{1}{t_{\text {acc }}}=\frac{\dot{\Sigma}}{\Sigma_{\text {layer }}} .
$$

The factor 3 is arbitrary, but reasonable. For an instability to have any noticeable effect on the system, it needs to grow in a time shorter than the lifetime $t_{\text {acc }}$ of the system. Also, our approach of treating the accreted layer as a quasi-steady system, and analysing the perturbations as if they occur in a time-steady system, is valid only if the time scale of the perturbations is sufficiently small compared to $t_{\text {acc }}$. For both reasons, the choice, $g_{\text {mode }}=3$, in equation (21) seems appropriate.

\subsection{Auxiliary Prescriptions}

The solution of the set of differential equations described in $§ 2.1$ requires knowledge of the thermodynamic and other physical properties of the gas. We describe here the particular prescriptions we have used for the calculations presented in this paper.

\subsubsection{Equation of State}

The pressure is assumed to be supplied by photons, nuclei and electrons. For the photons we use the blackbody formula and for the nuclei we assume an ideal non-degenerate gas. In the case of the electrons, we write the pressure as the quadrature sum of two terms, one equal to the pressure of an ideal non-degenerate gas and the other equal to the pressure of a zero-temperature degenerate electron gas. Thus we take (see Paczynski 1983b)

$$
\begin{gathered}
P=\frac{1}{3} a T^{4}+P_{\text {nuc }, \mathrm{nd}}+\left[P_{\mathrm{e}, \mathrm{nd}}^{2}+P_{\mathrm{e}, \mathrm{d}}^{2}\right]^{1 / 2}, \\
P_{\text {nuc }, \mathrm{nd}}=\frac{\rho k_{B} T}{\mu_{\mathrm{nuc}} m_{u}}, \quad P_{\mathrm{e}, \mathrm{nd}}=\frac{\rho k_{B} T}{\mu_{\mathrm{e}} m_{u}},
\end{gathered}
$$

where the molecular weights $\mu_{\text {nuc }}$ and $\mu_{\mathrm{e}}$ are determined in the standard way (e.g. Clayton 1983). In determining $\mu_{\text {nuc }}, \mu_{\mathrm{e}}$, we assume that a fraction $0.2 Z_{\text {out }}$ of the heavy elements in the accreted layer consists of ${ }^{56} \mathrm{Fe}$ and the rest (what we have called $Z_{\mathrm{CNO}}$, see eq. 2 ) consists of ${ }^{14} \mathrm{~N}$ (as a surrogate for $\mathrm{CNO}$ elements). The latter choice is an approximation. The initial CNO elements in the accreted gas consist mostly of ${ }^{12} \mathrm{C}$ and ${ }^{16} \mathrm{O}$. During $\mathrm{H}$-burning via the CNO cycle, the composition is mostly ${ }^{14} \mathrm{O}$ and ${ }^{15} \mathrm{O}$, while the composition reverts 
back to ${ }^{12} \mathrm{C}$ and ${ }^{16} \mathrm{O}$ after the $\mathrm{CNO}$ cycle is done. Considering the other simplifications we have employed, approximating the composition as ${ }^{14} \mathrm{~N}$ for the purposes of calculating the molecular weights seems reasonable. We take the stellar substrate below the accreted layer to be made of pure ${ }^{56} \mathrm{Fe}$.

For the zero-temperature degenerate electron pressure $P_{\text {deg }}$, we use an exact expression that is valid for all Fermi energies (Shapiro \& Teukolsky 1983). We do not include Coulomb corrections on the pressure. Separating the electron pressure into a non-degenerate part and a zero-temperature degenerate part and taking the quadrature sum is, again, an approximation. The separation works well in various asymptotic limits and is reasonably accurate even in the transition regime between non-degeneracy and degeneracy (Paczynski 1983b). We believe that the approximation is adequate for our purposes since the transition zone is usually fairly narrow in $\Sigma$.

The entropy term in equation (5) is important since it is the origin of the compressional flux. We write the entropy as the sum of contributions from each nuclear species and the electrons. The entropy per unit mass for species $i$ takes the form

$$
s_{i}=N_{i} k\left[-\ln \left(\rho N_{i}\right)+\frac{3}{2} \ln T+\frac{5}{2}+\frac{3}{2} \ln \left(\frac{2 \pi m_{i} k}{h^{2}}\right)\right],
$$

where $N_{i}$ is the number of particles of the particular species per unit mass of the gas, $m_{i}$ is the mass of each particle, and $h$ is Planck's constant. This expression corresponds to an ideal gas. In the case of the electrons, we use the above entropy so long as the quantity is positive, and replace it with zero when the expression becomes negative. This allows us to handle both the non-degenerate and degenerate limits adequately.

\subsubsection{Opacity}

We include radiative and conductive energy transfer, and model the opacity $\kappa$ as

$$
\frac{1}{\kappa}=\frac{1}{\kappa_{\text {rad }}}+\frac{1}{\kappa_{\text {cond }}}
$$

For the radiative opacity $\kappa_{\text {rad }}$, we use the formulae given in Appendix A of Schatz et al. (1999), using the analytic formulae of Antia (1993) to calculate the electron chemical potential.

To calculate the conductive opacity we have used the software of Potekhin (1999). Up to a density of $10^{9} \mathrm{~g} \mathrm{~cm}^{-3}$, we include the effects of impurities among the nuclei. Above this density we assume that the material is pure, and we interpolate between the two regimes. 
The density of the fuel layer is nearly always less than $10^{9} \mathrm{~g} \mathrm{~cm}^{-3}$, except for some rare helium bursts.

\subsubsection{Nuclear Energy Generation Rates}

For $\epsilon_{\mathrm{H}}$, we include the pp chain and the CNO cycle. Because the temperature of the burning material is on the order of $10^{8} \mathrm{~K}$, the CNO cycle usually dominates. We include fast-CNO burning, saturated CNO burning and electron capture reactions, as described in Mathews \& Dietrich (1984) and Bildsten \& Cumming (1998), and write the energy-generation rate as

$$
\epsilon_{\mathrm{H}, \mathrm{CNO}}=4 E_{\mathrm{H}}^{*} r_{\mathrm{CNO}} \frac{Z_{\mathrm{CNO}}}{14},
$$

where $r_{\mathrm{CNO}}$ is the rate of reactions per CNO nucleus, and we have assumed that in equilibrium the majority of the CNO nuclei are ${ }^{14} \mathrm{~N}$ (Clayton 1983). For the reaction rate, we assume

$$
r_{\mathrm{CNO}}=\left(\frac{1}{\tau_{13}}+\frac{1}{862.0 \mathrm{~s}}\right)^{-1}\left[\left(\frac{1}{\tau_{13}}\right)\left(\frac{1}{\tau_{13}+\tau_{14}+278.2 \mathrm{~s}}\right)+\left(\frac{1}{862.0 \mathrm{~s}}\right)\left(\frac{1}{\tau_{14}+1038.0 \mathrm{~s}}\right)\right] .
$$

Here, $\tau_{13}$ is the lifetime of ${ }^{13} \mathrm{~N}$ against the reaction ${ }^{13} \mathrm{~N}(p, \gamma){ }^{14} \mathrm{O}$ (Mathews \& Dietrich 1984),

$$
\begin{aligned}
\tau_{13}=\left(X \rho / \mathrm{g} \mathrm{cm}^{-3}\right)^{-1}\left[3.35 \times 10^{7} T_{9}^{-2 / 3} \exp \left(-15.202 T_{9}^{-1 / 3}-0.8702 T_{9}^{2}\right)\right. \\
\times\left(1+0.027 T_{9}^{1 / 3}+0.9 T_{9}^{2 / 3}+0.173 T_{9}+4.61 T_{9}^{4 / 3}+2.26 T_{9}^{5 / 3}\right) \\
\left.+3.03 \times 10^{5} T_{9}^{-3 / 2} \exp \left(-6.348 T_{9}^{-1}\right)\right]^{-1} \mathrm{~s},
\end{aligned}
$$

where $T_{9}=T /\left(10^{9} \mathrm{~K}\right)$, and $\tau_{14}$ is the lifetime of ${ }^{14} \mathrm{~N}$ against the reaction ${ }^{14} \mathrm{~N}(p, \gamma){ }^{15} \mathrm{O}(\mathrm{e} . \mathrm{g}$. Paczynski 1983a),

$$
\tau_{14}=3.1 \times 10^{10}\left(X \rho / \mathrm{gcm}^{-3}\right)^{-1} T_{6}^{2 / 3} \exp \left(152.313 T_{6}^{-1 / 3}\right) \mathrm{s},
$$

where $T_{6}=T /\left(10^{6} \mathrm{~K}\right)$. The time scale $862.0 \mathrm{~s}$ refers to the beta-decay timescale of ${ }^{13} \mathrm{~N}$, $278.2 \mathrm{~s}$ is the sum of the beta-decay timescales of ${ }^{14} \mathrm{O}$ and ${ }^{15} \mathrm{O}$, and $1038.0 \mathrm{~s}$ is the sum of the beta-decay timescales of ${ }^{13} \mathrm{~N}$ and ${ }^{15} \mathrm{O}$. In deriving the above rates we have assumed that the various species have reached their equilibrium abundances.

Figure 1 shows the variation of $\epsilon_{\mathrm{H}}$ and $\epsilon_{\mathrm{He}}$ with temperature for some typical densities. For low and moderate temperatures, the hydrogen-burning rate is a steeply increasing function of temperature, and it is this steep dependence that drives a thermonuclear instability. For a temperature greater than about $10^{7.8}-10^{7.9} \mathrm{~K}$, however, hydrogen-burning switches 
rather abruptly to the saturated burning regime. Beyond this temperature, hydrogenburning is stable. As we discuss in $\S 3$, this change has a noticeable effect on the sequence of equilibria. We have corrected the proton capture rates for screening using the formulae of Dewitt et al. (1973) for the non-resonant reactions and the formulae of Itoh et al. (2003) for the resonant reaction $\left({ }^{13} \mathrm{~N}(p, \gamma){ }^{14} \mathrm{O}\right)$. Screening increases the reaction rates at temperatures where the CNO cycle is not saturated.

For $\epsilon_{\mathrm{He}}$, we use the fitting formula given in equations (4.7), (4.8a) and (4.8b) of Fushiki \& Lamb (1987b) which include screening. We introduce a smooth transition between the various regimes defined by these authors in order to more faithfully reproduce the numerical results they have depicted in their Fig. 3. Our fitting results are shown in Fig. 1.

\section{Equilibrium Solutions}

Paczynski (1983a) has presented a very helpful analysis of the stability of nuclear burning on the surface of a compact star. His model involves numerous simplifications: he uses a onezone approximation, he considers only helium burning, and he assumes an inner boundary condition on the flux rather than on the temperature. Nevertheless, many of the insights he has obtained via his simple analysis carry over to our more detailed work. In particular, following his work, we have found it very helpful to consider equilibria in the $F_{\text {out }}-\Sigma_{\text {layer }}$ plane. Appendix A discusses why the insights from Paczyński's analysis apply to our more complicated model.

Figures 2 and 3 show sequences of equilibria of the accreted layer for a $1.4 M_{\odot}$ neutron star with a radius of $10.4 \mathrm{~km}$ and a core temperature of $10^{8} \mathrm{~K}$. The different panels correspond to different mass accretion rates $\dot{M}$, parameterized by the accretion luminosity $L_{\text {acc }}$ measured at infinity,

$$
L_{\mathrm{acc}}=\dot{M} c^{2} \frac{z}{(1+z)} \equiv l_{\mathrm{acc}} L_{\mathrm{Edd}}
$$

where $L_{\mathrm{Edd}}=4 \pi G M c / \kappa_{\mathrm{es}}$, with $\kappa_{\mathrm{es}}=0.4 \mathrm{~cm}^{2} \mathrm{~g}^{-1}$, is the Eddington luminosity measured at infinity. The local surface mass accretion rate $\dot{\Sigma}$ is related to $\dot{M}$ by

$$
\dot{\Sigma}=\frac{\dot{M}}{4 \pi R^{2}}(1+z) .
$$

The mass accretion rate $\dot{M}$, or equivalently $\dot{\Sigma}$, is a key parameter that determines the nature

of bursts. Since it is however not directly measured, we prefer to give all our results in terms of the dimensionless luminosity $l_{\text {acc }}=L_{\text {acc }} / L_{\text {Edd }}$. In doing this, we assume that the accretion is radiatively efficient and satisfies the relation given in equation (30). 


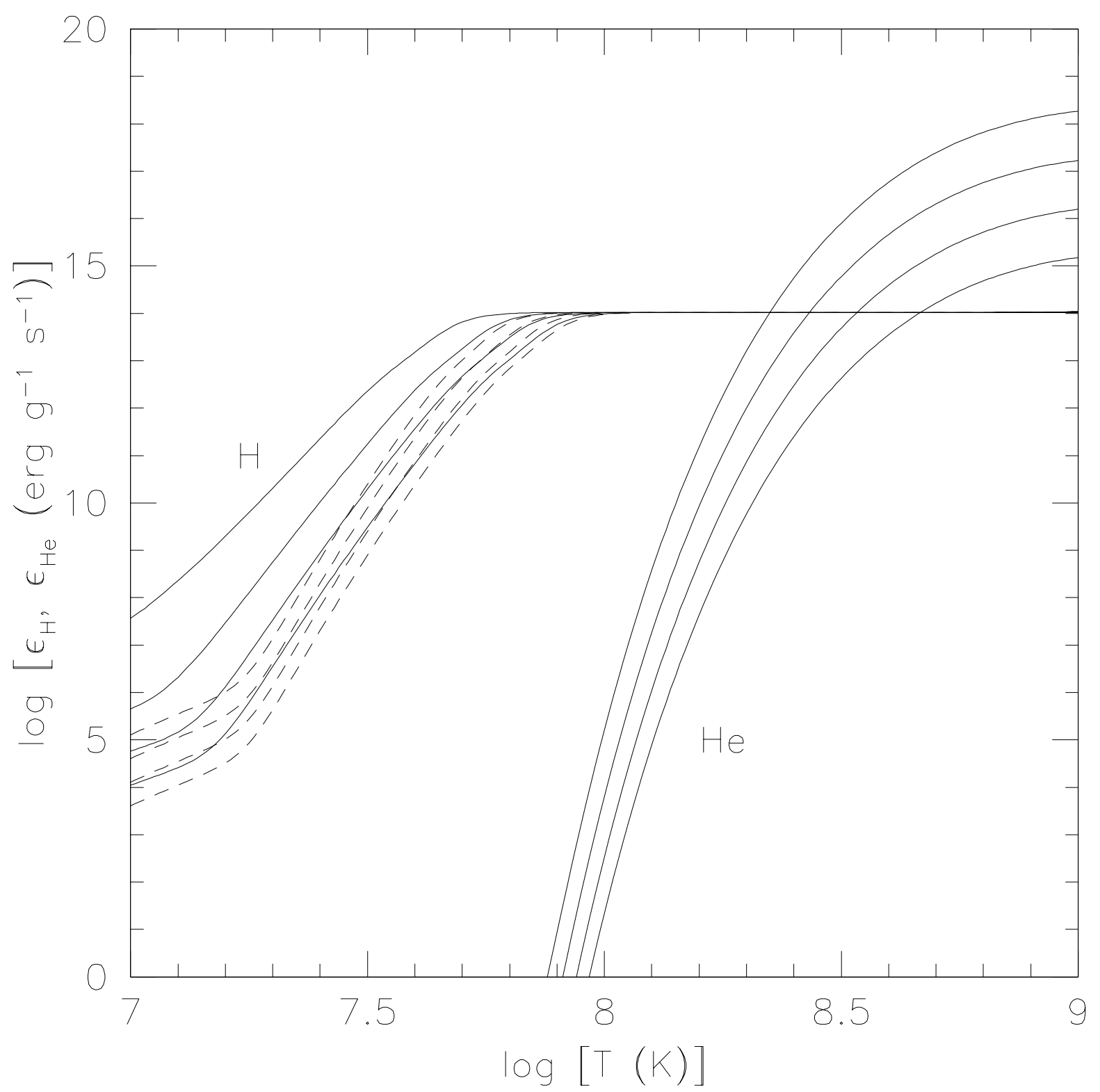

Fig. 1.- Nuclear reaction rates for hydrogen and helium, plotted as a function of temperature. From bottom to top, the four curves correspond to densities of $10^{5}, 10^{5.5}, 10^{6}, 10^{6.5} \mathrm{~g} \mathrm{~cm}^{-3}$. The hydrogen-burning curve is dominated by the pp chain at low temperatures, the $\mathrm{CNO}$ cycle at intermediate temperatures, and saturates above about $10^{7.8}-10^{7.9} \mathrm{~K}$. The dashed lines trace the results if one ignores screening. The helium rates are taken from Fushiki \& Lamb (1987b). 


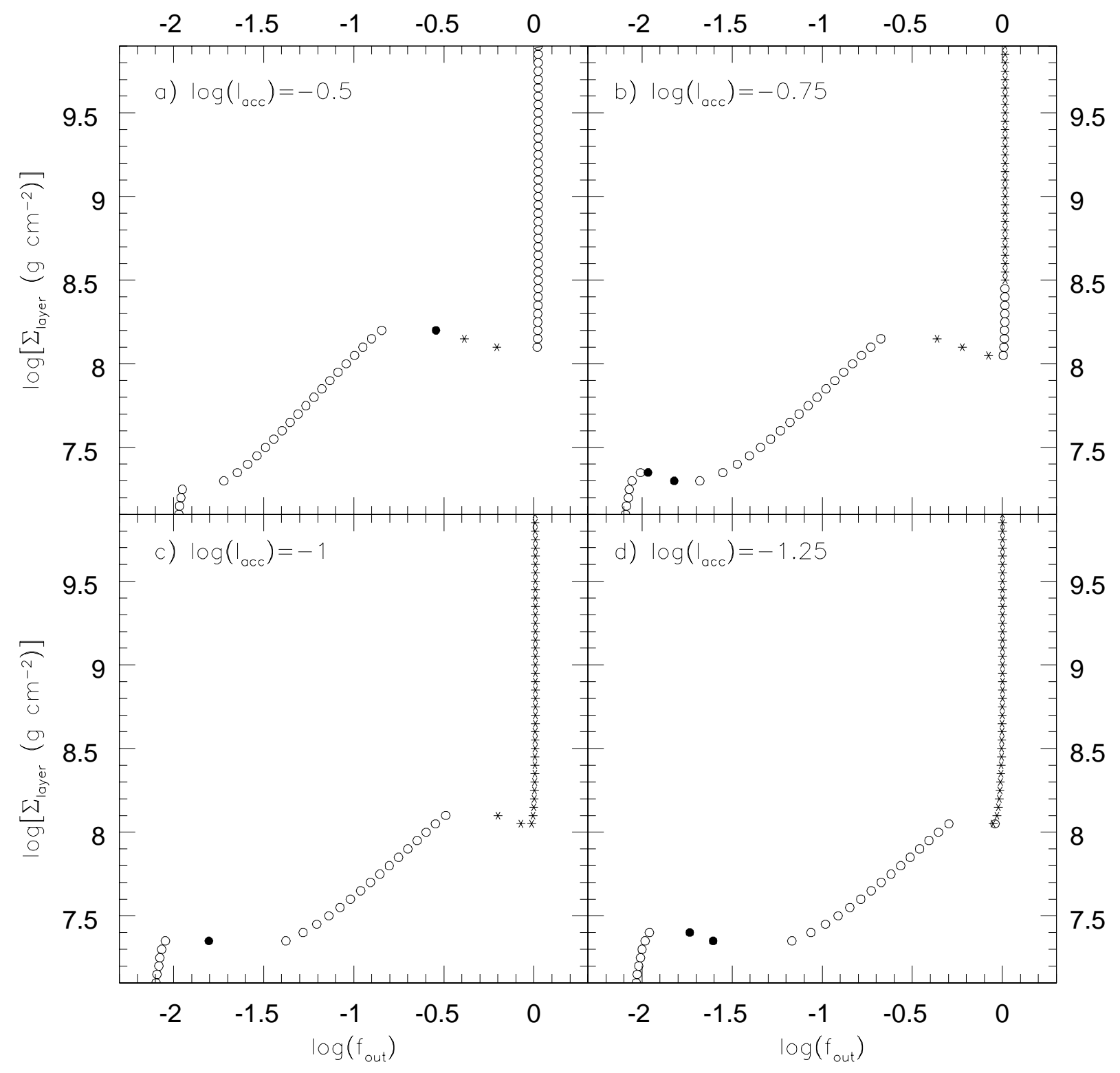

Fig. 2.- The points trace the locus of values of the surface density of the accreted layer $\Sigma_{\text {layer }}$ and the normalized escaping flux $f_{\text {out }}=F_{\text {out }} / F_{\text {nuc }}$ which satisfy the outer and inner boundary conditions. The calculations assume a neutron star of mass $M=1.4 \mathrm{M}_{\odot}$, radius $R=10^{0.4} R_{s}=10.4 \mathrm{~km}$, and core temperature $T_{\text {core }}=10^{8} \mathrm{~K}$. The four panels correspond to four relatively large accretion luminosities. An open circle indicates that the corresponding equilibrium is stable. A filled circle indicates that the equilibrium is unstable with a real mode frequency $\gamma$, while a star indicates an unstable equilibrium with complex $\gamma$, i.e., an overstability. 


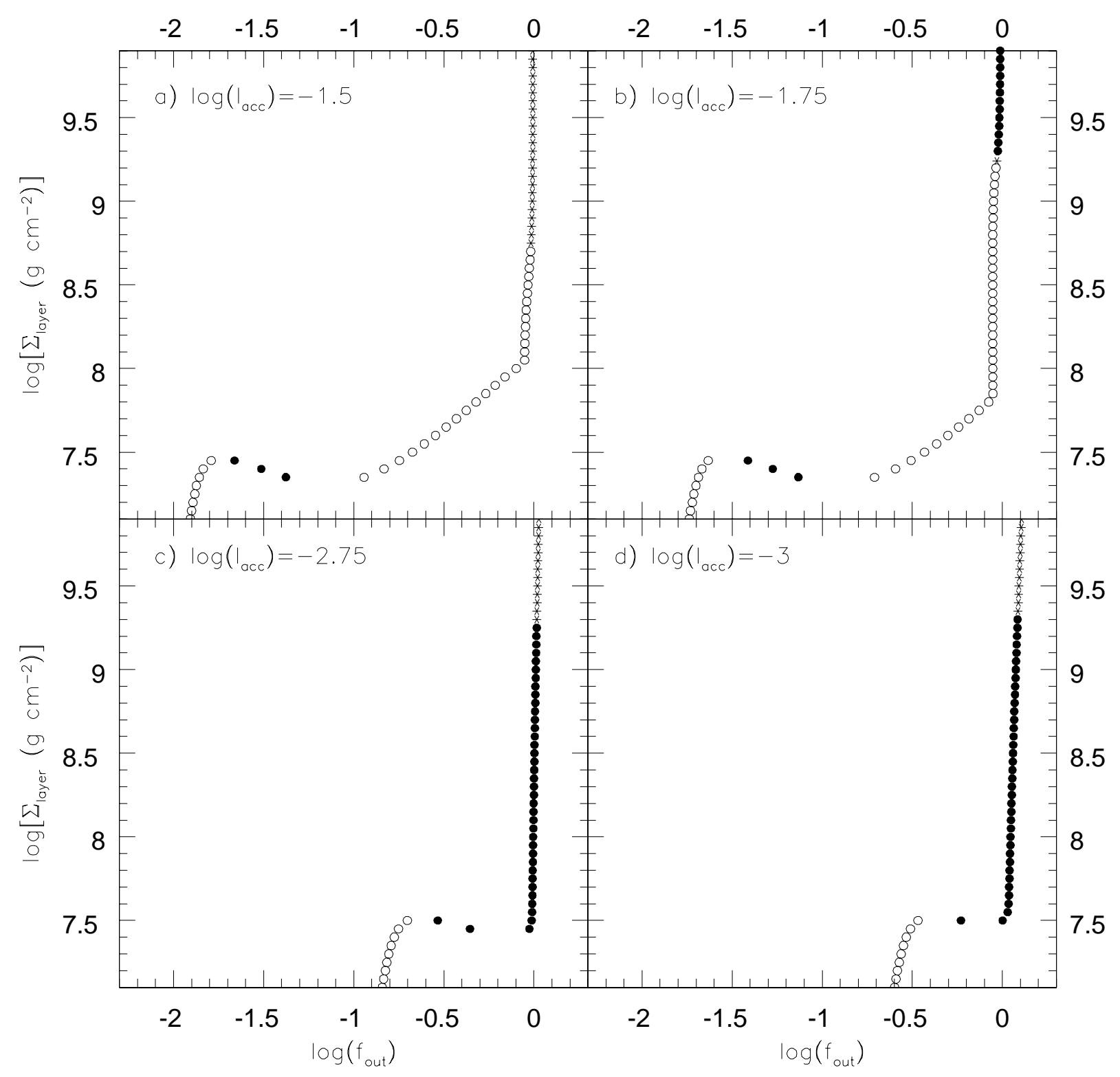

Fig. 3.- Similar to Fig. 2, but for four lower accretion luminosities. 
The eight panels in Figs. 2, 3 correspond to accretion luminosities $\log \left(l_{\text {acc }}\right)=-0.5$, $-0.75,-1,-1.25,-1.5,-1.75,-2.75$ and -3 in Eddington units. In each panel, the horizontal axis shows the escaping flux $f_{\text {out }}$ from the accreted layer, normalized by the maximum nuclear burning energy available in the accreting gas:

$$
f_{\text {out }} \equiv \frac{F_{\text {out }}}{F_{\text {nuc }}}, \quad F_{\text {nuc }}=\dot{\Sigma}\left[X_{\text {out }} E_{\mathrm{H}}^{*}+\left(X_{\text {out }}+Y_{\text {out }}\right) E_{\mathrm{He}}^{*}\right] .
$$

The vertical axis shows the column density of the layer, $\Sigma_{\text {layer }}$.

The most obvious feature of the various panels is that the equilibria do not form monotonic sequences in the $F_{\text {out }}-\Sigma_{\text {layer }}$ plane. At a given $\Sigma_{\text {layer }}$, there can be one, three, or even five, distinct solutions for $F_{\text {out }}$. (There are no cases of five solutions in the sequences shown here, but it is fairly common when the core temperature is lower, e.g., $10^{7.5} \mathrm{~K}$ ). Because the equations are nonlinear and include many different physical effects, it is not surprising to have multiple solutions.

Consider as an example Fig. 2(b), which corresponds to an accretion luminosity $\log \left(l_{\text {acc }}\right)=$ -0.75 . The sequence of equilibria shows two peaks, one at $\log \left(f_{\text {out }}\right) \sim-2$ and one at $\sim-0.5$. In addition, at the very right, there is a steep vertical segment which we refer to as the "wall." Fig. 4 shows other details of the equilibria for this choice of $l_{\text {acc }}$; panel (a) is a plot of $\Sigma_{\text {layer }}$ vs the temperature $T_{\text {layer }}$ at the base of the layer, while panel (b) shows the variation of the H-fraction $X_{\text {layer }}$, He-fraction $Y_{\text {layer }}$ and heavy element fraction $Z_{\text {layer }}$ at the bottom of the layer.

When the column depth of the accreted layer $\Sigma_{\text {layer }}$ is very small, the gas is neither hot nor dense and does not undergo any nuclear burning. Thus, $X_{\text {layer }}=X_{\text {out }}, Y_{\text {layer }}=$ $Y_{\text {out }}, Z_{\text {layer }}=Z_{\text {out }}$, and the flux $F_{\text {out }}$ that emerges is mostly what is released as a result of compressing the accreting gas, plus any flux that escapes from the $10^{8} \mathrm{~K}$ core. This stage corresponds to the nearly vertical segment at the left of Fig. 2(b). As $\Sigma$ increases, the gas at the bottom of the layer becomes hotter. Ultimately, when $\Sigma \sim 10^{7.3} \mathrm{~g} \mathrm{~cm}^{-2}$ and the temperature is about $10^{7.7} \mathrm{~K}$, hydrogen-burning begins. Soon after this, hydrogen-burning takes over as the dominant term in the energy equation, and at this point, the $\Sigma_{\text {layer }}-F_{\text {out }}$ curve in Fig. 2(b), as well as $\Sigma_{\text {layer }}-T_{\text {layer }}$ curve in Fig. 4(a), reverse direction, producing the peak on the left in the two plots. We identify this peak as the "hydrogen peak." The onset of hydrogen-burning is also evident in the run of $X_{\text {layer }}$ in Fig. 4(b) (see also $Y_{\text {layer }}$ whose variations simply reflect the amount of helium produced by hydrogen-burning).

As we descend from the top of the hydrogen peak in the direction of increasing $F_{\text {out }}$ and $T_{\text {layer }}$, a point is reached when hydrogen-burning becomes saturated (because the reactions are beta-limited). This happens around $T_{\text {layer }} \sim 10^{7.9} \mathrm{~K}$. Beyond this point, the sequence of equilibria start rising again in the $\Sigma_{\text {layer }}-F_{\text {out }}$ and $\Sigma_{\text {layer }}-T_{\text {layer }}$ planes. The rise continues for 

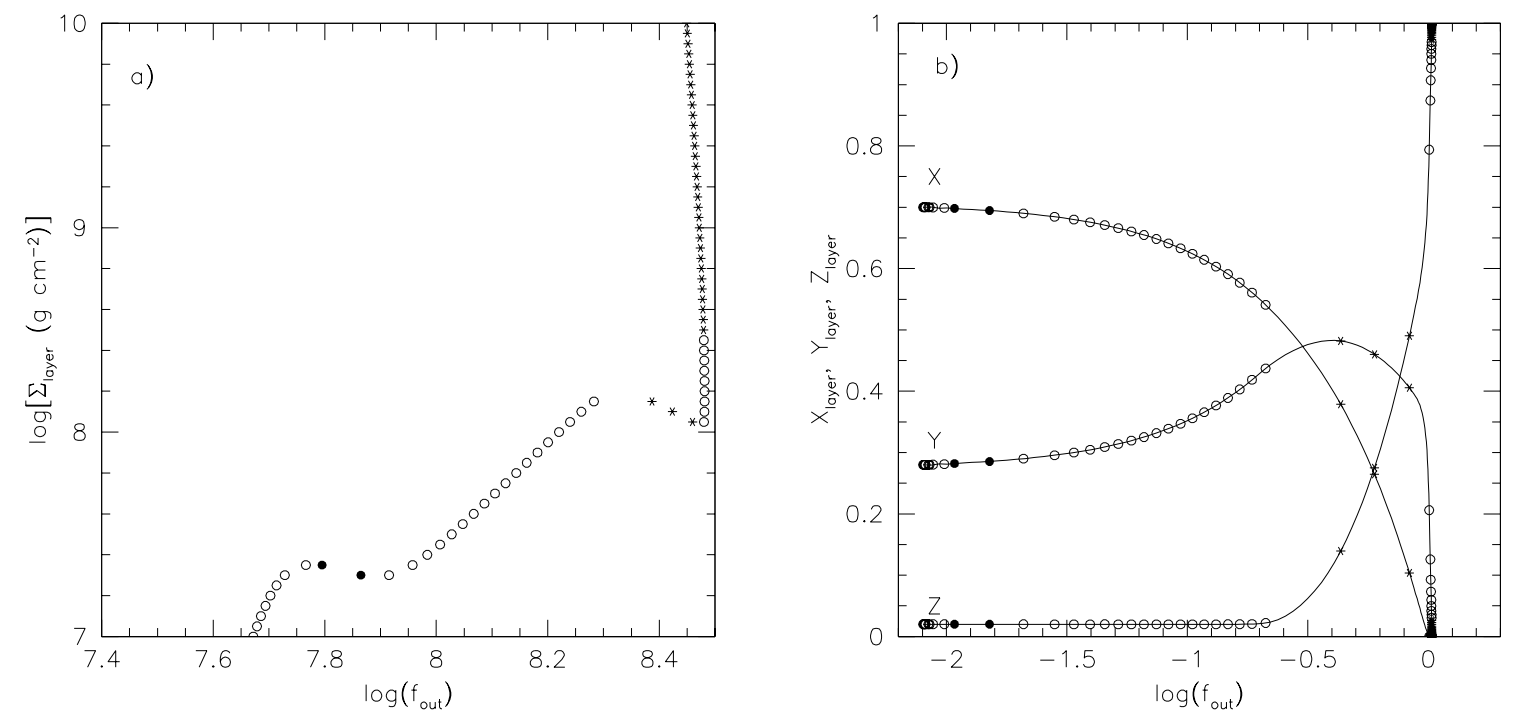

Fig. 4.- Additional details of the equilibrium sequence shown in Fig. 2b. (a) Shows the column density $\Sigma_{\text {layer }}$ of the accreted layer as a function of the temperature $T_{\text {layer }}$ at the bottom of the layer. The open circles, the filled circles and the stars have the same meaning as in Fig. 2. (b) Shows the mass fractions of hydrogen, helium and metals at the bottom of the layer as a function of the normalized outgoing flux. 
a while, with more and more hydrogen being burnt into helium until at $\Sigma_{\text {layer }} \sim 10^{8.1} \mathrm{~g} \mathrm{~cm}^{-2}$ and $T_{\text {layer }} \sim 10^{8.3} \mathrm{~K}$, helium-burning is initiated. At this point there is a second peak in the sequence of equilibria, the "helium peak."

As the sequence of equilibria fall off from the helium peak, the helium is burned rapidly, and the $\mathrm{CNO}$ elements that this produces cause the hydrogen-burning also to pick up since the rate of hydrogen burning is proportional to $Z_{\mathrm{CNO}}$ (eq. 26). When the hydrogen and helium are almost all exhausted, the sequence of equilibria turn round again and march up the wall. In this segment of the curve, very little changes as a function of increasing $\Sigma$. The hydrogen and helium are burned close to the surface within a column of order $10^{8} \mathrm{~g} \mathrm{~cm}^{-2}$, and the rest of the accreted layer consists just of burned CNO material which is progressively compressed by the weight of the overlying gas. Note that, since helium burning by the triple$\alpha$ reaction is not beta-limited, there is no regime of saturated helium burning. Therefore, the transition from the declining slope of the helium peak to the rapidly rising wall is generally quite abrupt. Figure 4(a) shows that, as $\Sigma_{\text {layer }}$ increases on the wall, the temperature at the base of the layer falls. This is because there is a net flux flowing from the layer into the star. The nuclear burning occurs at a fixed $\Sigma$ in this sequence of wall models. With increasing $\Sigma_{\text {layer }}$, there is a larger dead column between the burning layer and the base of the layer, and the ingoing flux causes the temperature at the base to drop.

We should note at this point that not all equlibria shown in Figs. 2-4 are accessible to a real system. Imagine starting with a bare neutron star and adding gas at the specified rate $\dot{\Sigma}$. As $\Sigma_{\text {layer }}$ increases, the system will ride up the left slope of the hydrogen peak. When $\Sigma_{\text {layer }}$ is equal to the maximum $\Sigma \sim 10^{7.3} \mathrm{~g} \mathrm{~cm}^{-2}$ of the hydrogen peak, the system no longer has any equilibria available in the vicinity of the peak. Therefore, it will relax towards the nearest available equilibrium, which is a solution with the same value of $\Sigma_{\text {layer }} \sim 10^{7.3} \mathrm{~g} \mathrm{~cm}^{-2}$ on the left slope of the helium peak. Thus, all the equilibria on the right slope of the hydrogen peak and the lower left slope of the helium peak will be by-passed, since they correspond to lower values of $\Sigma_{\text {layer }}$ than the current layer thickness. With increasing $\Sigma_{\text {layer }}$, the system will ride up the helium peak until it reaches the maximum $\Sigma \sim 10^{8.1} \mathrm{~g} \mathrm{~cm}^{-2}$ of the helium peak. At this point, the system will once again move across to the nearest available solution, which is located on the wall, bypassing the equilibria in the valley to the right of the helium peak. Having reached the wall, the system will continue rising up the wall with increasing $\Sigma_{\text {layer }}$.

Let us turn now to the other sequences of equilibria shown in Figs. 2 and 3. Fig. 2(a) corresponds to $\log \left(l_{\text {acc }}\right)=-0.5$. In this case, because of the rapid accretion rate, the accreted layer is quite hot, and so hydrogen burning is already in the saturated limit when it turns on. There is, therefore, no hydrogen peak (though there is the semblance of a plateau). Fig. 2(b) was discussed above, and has a modest hydrogen peak. Note that the helium peak in Fig. 
2(b) is pushed to the right relative to the peak in Fig. 2(a). This is because helium requires a temperature of at least about $2 \times 10^{8} \mathrm{~K}$ to burn (Fig. 1), and such high temperatures are obtained at the lower accretion rate only at larger values of $F_{\text {out }}$. With decreasing accretion rate, the gas temperature continues to fall and the hydrogen peak becomes more pronounced (Figs. 2c,d). The helium peak also gets pushed progressively farther to the right, until it finally hits the wall and disappears. For accretion luminosities below about $10^{-1.25} L_{\text {Edd }}$, there is no helium peak. Helium now starts burning on the wall, at progressively larger values of $\Sigma_{\text {layer }}$ with decreasing $l_{\text {acc }}$.

The above results are for a core temperature of $T_{\text {core }}=10^{8} \mathrm{~K}$. The pattern of peaks can be different for other core temperatures. If the core temperature is a few times $10^{8} \mathrm{~K}$, the hot core keeps the accreted layer hot under all circumstances and hydrogen burning is always in the saturated regime. In this case, there is no separate hydrogen peak. On the other hand, for lower values of $T_{\text {core }}$, e.g. a few times $10^{7} \mathrm{~K}$, the hydrogen peak becomes quite pronounced and also has a higher peak value of $\Sigma_{\text {layer }}$. In this case, it is often possible to have situations in which the helium peak is lower than the hydrogen peak. If such is the case, when the system reaches the top of the hydrogen peak it will move directly across to the wall without stopping at the helium peak.

\section{Stability}

The previous section described sequences of equilibria for various mass accretion rates. As explained in $\S 2$, for every equilibrium we carry out a linear stability analysis to check whether the system is stable or unstable (the latter is determined by the criterion given in eq. 21). In Figs. 2 and 3, open circles indicate stable equilibria, filled circles indicate unstable equilibria in which the most unstable mode has a real $\gamma \geq 3 \gamma_{\text {acc }}$, and stars indicate unstable equilibria in which the fastest-growing mode has a complex $\gamma$, with $\Re(\gamma) \geq 3 \gamma_{\text {acc. }}$.

From the various panels in Figs. 2 and 3, we quickly discern the following patterns. The left slopes of the hydrogen and helium peaks always correspond to stable equilibria, while the right slopes of both are always unstable; this is similar to the pattern described by Paczynski (1983a, who only considered He-burning) and is easily understood from his onezone analysis. As explained in Appendix A, the same pattern is expected in our problem as well. The stability of equilibria on the wall is variable. Sometimes the equilibria on the wall are fully stable, sometimes they are fully unstable, and sometimes some equilibria are stable and some are unstable. As we discuss now, it is the stability of the wall that determines whether or not a particular accretion rate leads to thermonuclear bursts. 
Consider as an example the case shown in Fig. 2(b), corresponding to $\log \left(l_{\text {acc }}\right)=-1$. Starting from the bare neutron star, as gas piles up, the system moves up the hydrogen peak along a sequence of equilibria that are all stable (open circles). When $\Sigma_{\text {layer }}$ hits the top of the hydrogen peak, the system moves across to a point on the left slope of the helium peak with the same value of $\Sigma_{\text {layer }}$. There is some adjustment of the accreted layer in order to switch to the new solution, but because the equilibrium solution here is stable the system is able to make the adjustment. With increasing $\Sigma_{\text {layer }}$, the system climbs up the helium peak along a sequence of stable equilibria. As before, once it reaches the top of the peak, it moves across to the right, this time to the wall, and the solution here is again stable. However, as the system climbs up the wall, it reaches a point at which the equilibrium is unstable. At this point, there is no stable equilibria available for any value of $f_{\text {out }}$. The system therefore undergoes a thermonuclear burst.

We may now quickly identify which of the other cases shown in Fig. 2 and 3 are stable and which unstable. The high accretion rate system in Fig. 2(a) is stable because the states on the wall are all stable. The system will move across to the wall after it hits the helium peak, and it will then climb up the wall along a sequence of stable equilibria to arbitrarily

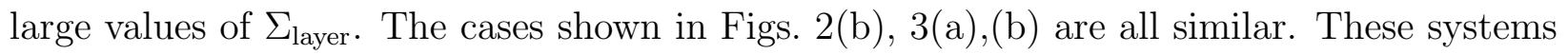
reach the wall stably, climb part way up the wall and then go unstable; we call all these cases "delayed bursts." In contrast, the cases shown in Figs. 2(c),(d), 3(c),(d) become unstable the moment they hit the top of the last peak since there are no stable equilibria available on the wall; we call these "prompt bursts."

\subsection{Unstable vs Overstable Modes}

We pause to discuss the distinction between modes with real $\gamma$ and those with complex $\gamma$. The former correspond to a simple instability, where the mode amplitude grows exponentially with time, whereas the latter correspond to an overstability, where the mode oscillates at a frequency equal to the imaginary part of $\gamma$ even as the amplitude grows. As Figs. 2 and 3 show, we see both kinds of behavior for our unstable modes. We should note that complex eigenvalues $\gamma$ always appear in complex conjugate pairs, which is obvious from the structure of the governing equations.

As explained in Appendix A, from an inspection of the steady state and linear perturbation equations, it can be seen that any system that is at the top of a peak or the bottom of a valley, i.e., where $d \Sigma_{\text {layer }} / d F_{\text {out }}=0$, has a zero-frequency mode, $\gamma=0$. Once we recognize this important rule, we can understand why the right slopes of the hydrogen and helium peaks are unstable. The argument goes as follows: the left slopes are stable, with all modes 
having $\Re(\gamma)<0$; the peak has a mode with $\gamma=0$; by continuity, this particular mode should have $\gamma>0$ on the right slope and should be real.

The above rule has a corollary: if the system makes a transition from stability to instability at a point where $d \Sigma_{\text {layer }} / d F_{\text {out }} \neq 0$, then the unstable mode at this point must have a complex $\gamma$. This rule applies, for instance, to all the systems that have delayed bursts, i.e., become unstable half-way up the wall, e.g., Figs. 2(b), 3(a),(b). In these cases, when the system moves into the unstable zone, it always first hits an overstable region. The overstability may in some cases then become a pure instability as a pair of modes with complex $\gamma$ merge to spin off a pair of real $\gamma$. Also, it is not possible to tell "how complex" the mode is, i.e., the relative magnitudes of $\Re(\gamma)$ and $\Im(\gamma)$.

Finally, in cases where the wall is entirely unstable, we have not been able to find any helpful rule. Whether the instability corresponds to real or complex $\gamma$ can be determined only with a full calculation.

\subsection{Burst Energetics and Trigger Mechanism}

Because there are two burning fuels, hydrogen and helium, we would like to know which fuel determines the burst properties. There are two aspects to this question.

First, once an instability has been triggered, we would like to know how much energy is available for the burst in unburnt helium compared to the energy in unburnt hydrogen. Figure 5 shows this quantity as a function of accretion rate for three choices of the neutron star radius (assuming a mass of $1.4 M_{\odot}$ ) and four core temperatures. We have assumed that all the energy in the unburnt fuel is emitted as radiation during the burst. In actuality, some energy is lost into the core and some comes out as neutrinos; we neglect this complication.

In interpreting the results, we should note that hydrogen-burning to iron provides nearly 5 times more energy per gram than helium-burning to iron. Keeping this in mind, we see that for high mass accretion rates roughly in the range $\log \left(l_{\text {acc }}\right) \sim-0.6$ to -1.5 (the precise values depend on the neutron star radius and core temperature), the bursts are of a mixed kind with energy being contributed by both hydrogen and helium. For intermediate accretion rates, $\log \left(l_{\text {acc }}\right) \sim-1.5$ to -2.5 , we have pure helium-burning bursts. Finally, for $\log \left(l_{\text {acc }}\right) \lesssim-2.5$, we again have mixed bursts. Actually, this last range corresponds to pure hydrogen bursts and it is not clear if helium burning will be triggered during the burst. In making our

estimates we assumed that all the unburnt fuel is consumed. The ranges of $\log \left(l_{\text {acc }}\right)$ given here correspond to a core temperature of $10^{8} \mathrm{~K}$ and a neutron star radius of $10.4 \mathrm{~km}$. 


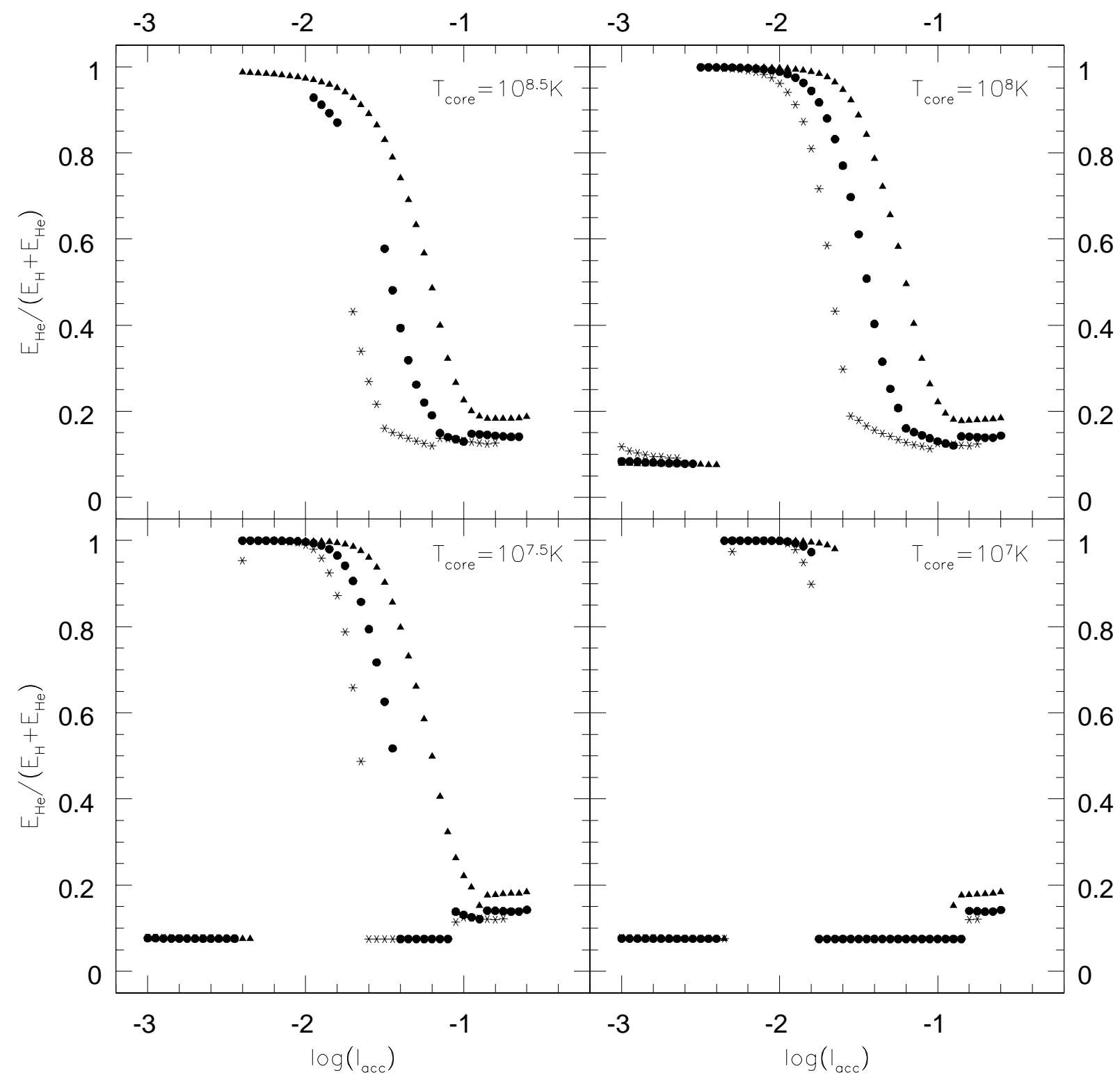

Fig. 5.- Ratio of the fluence in the burst due to helium burning to the fluence from hydrogen burning, assuming that each fuel is burned to ${ }^{56} \mathrm{Fe}$. The four panels correspond to core temperatures $T_{\text {core }}=10^{8.5}, 10^{8}, 10^{7.5}$ and $10^{7} \mathrm{~K}$, respectively. In each panel, the triangles, circles and stars trace the results for a neutron star of mass $1.4 M_{\odot}$ and radii $R=10^{0.6} R_{S}=16.4 \mathrm{~km}, 10^{0.4} R_{S}=10.4 \mathrm{~km}$, and $10^{0.2} R_{S}=6.5 \mathrm{~km}$, respectively. Note that hydrogen and helium burning both contribute at high and low luminosities (with hydrogen being about five times more important energetically per unit mass), while helium burning dominates at intermediate luminosities $\log \left(l_{\text {acc }}\right) \sim-1.5$ to -2.5 . 
Another interesting question is to understand what precisely triggers the burst instability. One way to do this is to look at the maximum temperature in the fuel layer (Fig. 6) and to thereby identify which fuel might be important. As we discussed in $\S 2.3$, hydrogen burning becomes saturated, and hence stable, for temperatures above about $10^{7.9} \mathrm{~K}$ (see Fig. 1). Therefore, we expect hydrogen-triggered bursts at lower temperatures and heliumtriggered bursts at higher temperatures. From Fig. 6 we see that the former occur at lower accretion rates and the latter at higher accretion rates. However, there is no clear way to distinguish the kinds of bursts from this plot since the variation of $T_{\max }$ with accretion luminosity is smooth. In our models, we do not find unstable modes for layers with maximum temperatures above $3.5 \times 10^{8} \mathrm{~K}$. This temperature limit is reached at $\log \left(l_{\text {acc }}\right) \sim-0.6$.

Gaining a deeper understanding of what triggers the bursts requires an examination of the eigenfunctions of the unstable modes. Specifically, we compute the contribution of helium burning integrated over the eigenfunction and take the ratio of this to the hydrogen burning integrated over the eigenfunction (here we do not consider the additional burning to iron as we did with the burst fluence ratio). The ratio should be large for a pure heliumtriggered burst and small for a pure hydrogen-triggered burst, and on the order of $0.1-0.2$ for mixed-triggered burst (the ratio is not 0.5 in this case since hydrogen-burning releases nearly an order of magnitude more energy per gram than helium-burning).

Figure 7 shows the above ratio as a function of $\log \left(l_{\mathrm{acc}}\right)$ for the same three choices of the neutron star radius and four choices of the core temperature. We see that, at high mass accretion rates $\log \left(l_{\text {acc }}\right) \sim-0.6$ to -1.5 , the bursts are triggered by both hydrogen and helium. Within this range, the higher accretion rates give delayed bursts, while the lower rates give prompt bursts (as seen from Fig. 2). For intermediate accretion rates $\log \left(l_{\text {acc }}\right) \sim-1.5$ to -2.5 , we have pure helium-triggered bursts. These all involve overstable modes. Finally, for $\log \left(l_{\text {acc }}\right)<-2.5$, we have hydrogen-triggered bursts. These are pure unstable modes (real $\gamma$ ).

Figures 8-9 show a typical eigenmode corresponding to $\log \left(l_{\mathrm{acc}}\right)=-0.9$. Near the surface $(\Sigma \rightarrow 0)$, all perturbations vanish according to our boundary conditions, except the flux, which is given a unit perturbation (in arbitrary units - it merely sets the normalization). The accreted layer extends up to $\Sigma_{\text {layer }}$, which is indicated by the thick vertical line. The eigenfunction then continues on into the substrate to a depth such that the diffusion time to that point is equal to twice the mode time scale (see $\S 2.2$ ). Note that, even though the inner boundary condition corresponds to a vanishing temperature perturbation, in fact the flux perturbation also vanishes at this point. This shows that our choice of the inner boundary is physically well-motivated. We have tried integrating the eigenmode to larger or smaller depth in the substrate and generally obtained the same results. 


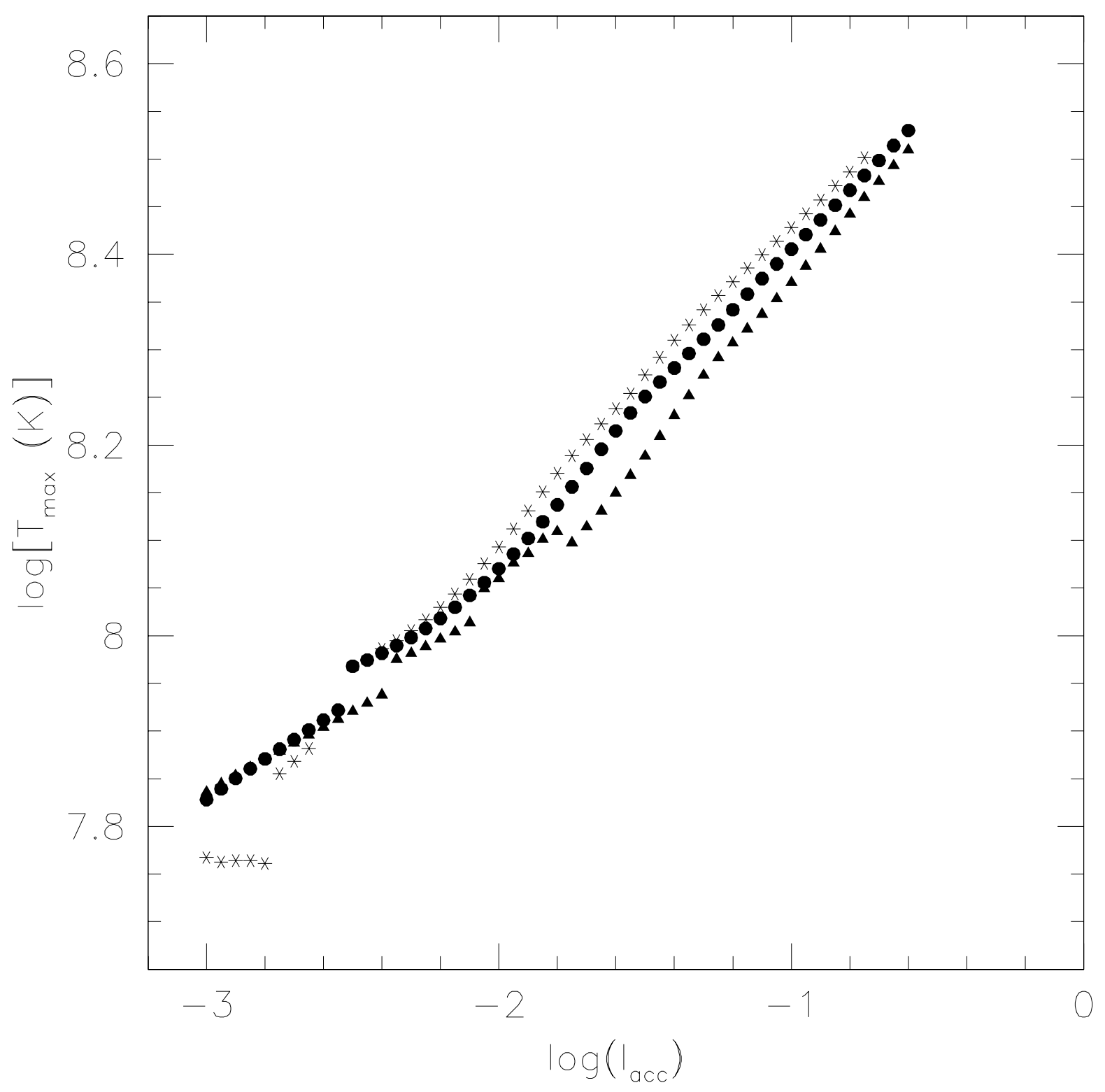

Fig. 6. - The maximum temperature $T_{\max }$ achieved in the accreted layer when the layer first becomes unstable, plotted as a function of the accretion luminosity. The core temperature is assumed to be $T_{\text {core }}=10^{8} \mathrm{~K}$, and the calculations correspond to the equilibrium at the "wall." The symbols have the same meanings as in Fig. 5. 


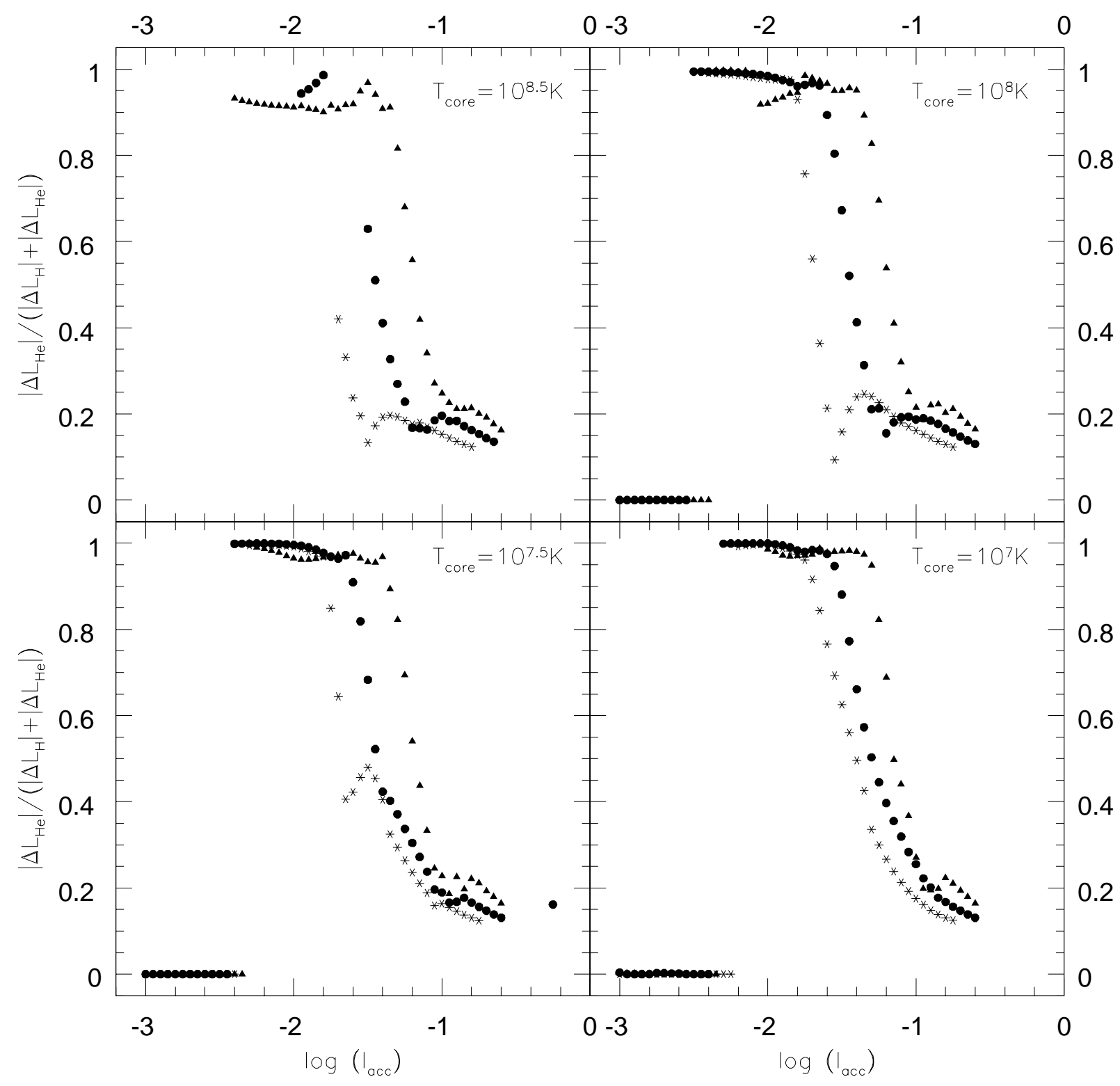

Fig. 7.- Ratio of the energy produced by helium burning in the linear eigenmode to the energy produced by hydrogen burning. The symbols and the layout are the same as in Fig. 5. Note that at high luminosities there is mixed burning with significant contributions from both hydrogen and helium, at intermediate luminosities helium burning dominates, while at low luminosities hydrogen burning dominates. 

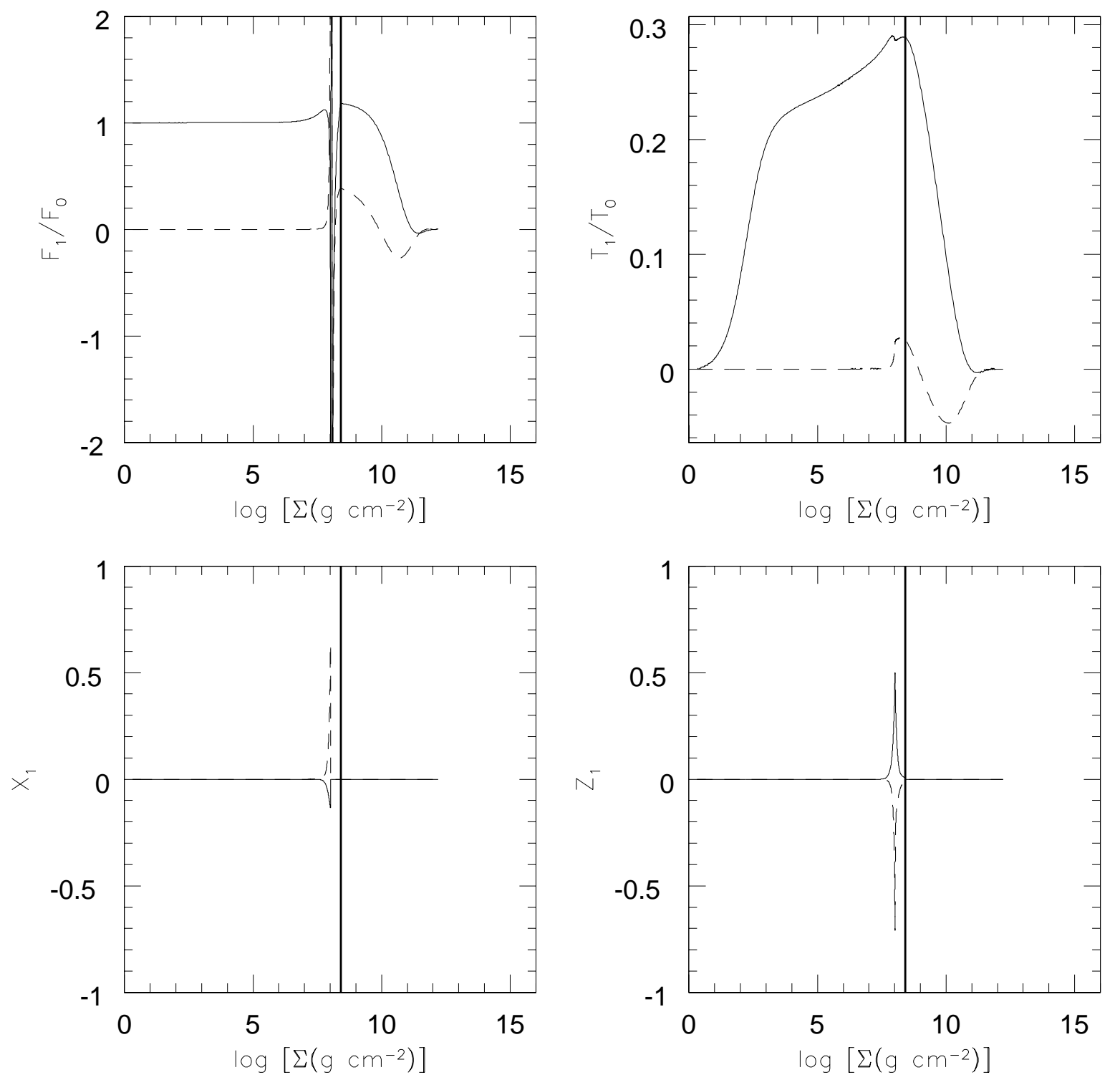

Fig. 8.- Overstable eigenmode for $\log \left(l_{\text {acc }}\right)=-0.9, M=1.4 M_{\odot}, R=10.4 \mathrm{~km}$ and $T_{\text {core }}=$ $10^{8} \mathrm{~K}$. The solid and dashed lines trace the real and imaginary parts of the mode. The bold vertical line shows the bottom of the accreted layer at $\Sigma=\Sigma_{\text {layer }}$. The eigenmode continues into the substrate to a depth $\Sigma_{\max }$, at which point the amplitude of the mode is substantially decreased. The boundary condition at $\Sigma_{\max }$ is that the temperature perturbation should vanish. However, it is seen that the perturbations in essentially all variables vanish there. 

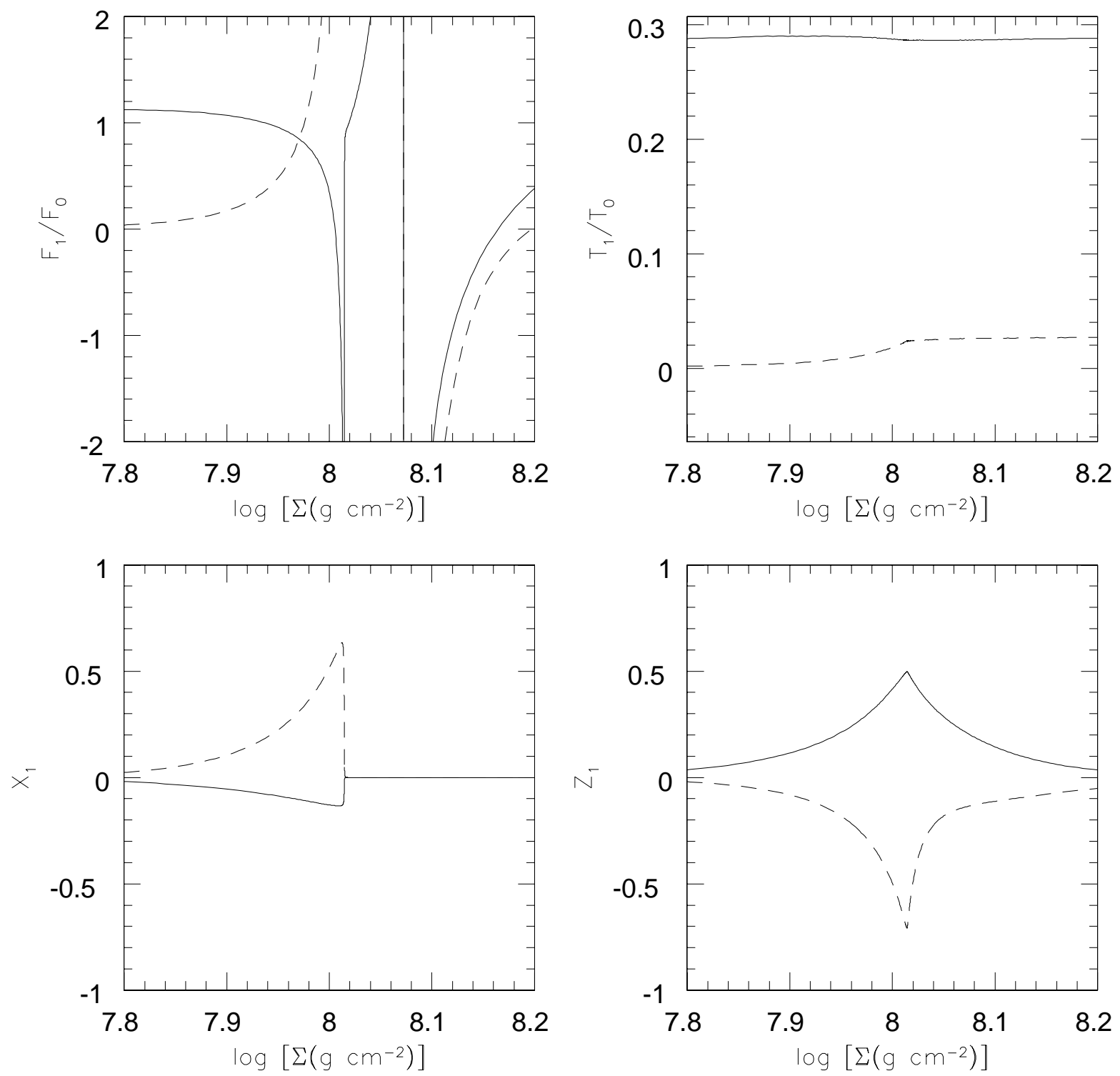

Fig. 9.- Expanded version of the eigenmode in Fig. 8, showing the burning layer in more detail. 
Within the accreted layer, the finite flux perturbation at the surface causes a temperature perturbation over the entire layer. However, the perturbations in $X$ and $Z$ are limited to a very narrow layer. This is the burning layer, which is shown in an expanded view in Fig. 9. The fluctuation in $X$ indicates hydrogen burning and the fluctuation in $Z$ corresponds to helium burning. For this mode, we see that both fuels burn at roughly the same depth, indicating that it is an example of a mixed burst. Also, since the eigenmode has nontrivial real and imaginary parts, we have a complex eigenvalue $\gamma$. In this particular case, the real part of $\gamma$ is equal to $8.9 \times 10^{-5} \mathrm{~s}^{-1}$ and the imaginary part is equal to $6.6 \times 10^{-4} \mathrm{~s}^{-1}$.

At lower accretion rates, the eigenmodes tend to be simpler. If we consider the critical values of $\Sigma_{\text {layer }}$ at which the systems shown in Figs. 3(a),(b) become unstable, the burning is dominated by helium and there is only a small contribution from hydrogen. Moreover, the two fuels tend to burn at different values of $\Sigma$. The eigenmode is still complex, though the imaginary part is not very large. If we go to yet lower accretion rates as in Figs. 3(c),(d), then there is no helium burning at all and the burning layer is dominated by hydrogen. In this case, the eigenmode is fully real.

\subsection{Putting Things Together}

Pulling together the various ideas discussed above, we identify five regimes of bursting behavior as a function of decreasing accretion luminosity. In the following, the numerical values correspond to the particular calculations presented earlier, in which $M=1.4 M_{\odot}$, $R=10^{0.4} R_{S}=10.4 \mathrm{~km}, T_{\text {core }}=10^{8} \mathrm{~K}$. Tables 2 and 3 give results for other selected choices of $R$ and $T_{\text {core }}$.

I. For very high accretion rates, with $\log \left(l_{\mathrm{acc}}\right)>-0.6$, there are no thermonuclear bursts. This is the regime of stable accretion, where the accreting gas is able to burn the nuclear fuel without instability.

II. For somewhat lower accretion rates $\log \left(l_{\text {acc }}\right) \sim-0.6$ to -0.85 , we have mixed bursts triggered by both hydrogen and helium. These bursts burn substantial amounts of both fuels. In this particular accretion range, the instability is delayed and is triggered only after the system climbs part way up the wall. We call these "delayed mixed bursts." As the system climbs up the wall, a large fraction of the nuclear fuel is burned stably in the accreted layer before the burst is triggered. ${ }^{4}$ As we shall see, this has dramatic consequences for both the

\footnotetext{
${ }^{4}$ Note that, on the wall, $f_{\text {out }}$ is nearly equal to unity, which means that the escaping flux in the equilibrium solution is almost equal to the nuclear energy content of the new fuel being added to the surface.
} 
recurrence times of the bursts and the ratio of the burst fluence to the total accretion fluence between bursts. When the system becomes unstable, the mode has a complex $\gamma$, which corresponds to an overstability. Thus, we expect the system to show an oscillatory behavior as it approaches the instability.

III. For yet lower accretion rates $\log \left(l_{\text {acc }}\right) \sim-0.9$ to -1.25 , we continue to have mixed triggered bursts burning mixed fuel. However, here the entire wall is unstable and so the instability is triggered when the system reaches the top of the helium peak. Because the burst happens as soon as the system hits the wall, we refer to these as "prompt mixed bursts," to distinguish them from the previous class of delayed mixed bursts. When the instability first begins at the peak, $\gamma$ is real, but when the system reaches the wall, $\gamma$ may possibly be complex (though not always). We cannot tell from our present analysis whether or not the system will show oscillatory behavior prior to the burst.

IV. For still lower accretion rates $\log \left(l_{\text {acc }}\right) \sim-1.3$ to -2.5 , bursts are again triggered only after the system climbs part way up the wall, i.e., we have delayed bursts. Here the instability is helium-triggered and the burst is dominated by helium burning. These correspond to the classic "helium bursts" that have been studied by previous authors. According to our calculations, $\gamma$ is complex when these systems go unstable, and this means that there should be some kind of oscillatory behavior prior to the burst. In practice, the imaginary parts of $\gamma$ tend to be small for these modes.

V. Finally, for the lowest accretion rates $\log \left(l_{\text {acc }}\right)<-2.5$, the wall is again unstable, and now the burst happens as soon as the system hits the top of the hydrogen peak. The instability is triggered by hydrogen burning, and we call these "hydrogen bursts." They do not have any oscillatory behavior. It is not clear if these hydrogen bursts will trigger helium burning. If they do not, then the helium will accumulate as the ashes of hydrogen burning and the system is likely to have enormous but very rare helium bursts.

The various regimes described above are summarized in Tables 2, 3. Much of our discussion in the rest of the paper is in terms of the burst regimes discussed in this section. Many of the regimes have been recognized by previous authors (Fujimoto et al. 1981; Fushiki \& Lamb 1987a; Bildsten 2000), but one of the new ideas to come out of our analysis is the distinction between delayed mixed bursts and prompt mixed bursts. This distinction has not been made earlier. The other new aspect is our ability to distinguish between simple exponential instability and oscillatory overstability. It is unique to our work since this is the

Nevertheless, the accreted layer does possess energy in unburnt fuel. Loosely speaking, it is the unburnt fuel that accumulated in the layer prior to reaching the wall. The energy from burning this fueld is released during the burst. 
Table 2. Burst Regimes for $T_{\text {core }}=10^{7.5} \mathrm{~K}$

\begin{tabular}{lllll}
\hline \hline \multicolumn{4}{c}{ Range in $\log \left(l_{\text {acc }}\right)$} \\
\multicolumn{1}{c}{$R=6.5 \mathrm{~km}$} & \multicolumn{1}{c}{$R=10.4 \mathrm{~km}$} & \multicolumn{1}{c}{ Type of Burst } & Symbol in Fig. 10 \\
\hline I. & $\geq(-0.7)$ & $\geq(-0.55)$ & No Bursts & None \\
II. & $(-0.75)$ to $(-1.0)$ & $(-0.6)$ to $(-0.85)$ & Delayed Mixed & Naked Star \\
III. & $(-1.05)$ to $(-1.6)$ & $(-0.9)$ to $(-1.4)$ & Prompt Mixed & Circled Symbol \\
IV. & $(-1.65)$ to $(-2.3)$ & $(-1.45)$ to $(-2.4)$ & (Delayed) Helium & Naked Star \\
V. & $\leq(-2.35)$ & $\leq(-2.45)$ & (Prompt) Hydrogen & Circled Symbol \\
\hline
\end{tabular}

Table 3. Burst Regimes for $T_{\text {core }}=10^{8} \mathrm{~K}$

\begin{tabular}{lllll}
\hline \hline \multicolumn{4}{c}{ Range in $\log \left(l_{\text {acc }}\right)$} \\
& \multicolumn{1}{c}{$R=6.5 \mathrm{~km}$} & \multicolumn{1}{c}{ Type of Burst } & Symbol in Fig. 10 \\
\hline I. $\quad \geq(-0.7)$ & $\geq(-0.55)$ & No Bursts & None \\
II. & $(-0.75)$ to $(-1.0)$ & $(-0.6)$ to $(-0.85)$ & Delayed Mixed & Naked Star \\
III. & $(-1.05)$ to $(-1.4)$ & $(-0.9)$ to $(-1.25)$ & Prompt Mixed & Circled Symbol \\
IV. & $(-1.45)$ to $(-2.4)$ & $(-1.3)$ to $(-2.5)$ & (Delayed) Helium & Naked Star \\
V. & $\leq(-2.65)$ & $\leq(-2.55)$ & (Prompt) Hydrogen & Circled Symbol \\
\hline
\end{tabular}


first study to carry out a full linear stability analysis to calculate eigenmodes and (complex) eigenfrequencies.

The other comment we should make is that the pattern of five regimes described above is valid only for intermediate core temperatures $\sim 10^{7.5}-10^{8} \mathrm{~K}$. We have focused on this case since it represents the likely core temperature for standard neutrino cooling models of

accreting neutron stars. For higher core temperatures, e.g., $10^{8.5} \mathrm{~K}$, the hydrogen-burning layer is hotter than $10^{7.8} \mathrm{~K}$ so that the systems are in the saturated hydrogen-burning limit. They therefore lack the hydrogen-triggered bursts at low accretion rates. On the other hand, if the core temperature is lower, e.g., $10^{7} \mathrm{~K}$, the prompt mixed bursts are no longer triggered by the helium peak. Instead, the bursts are triggered when the accreted layer reaches the top of the hydrogen peak, which is now higher than the helium peak. The bursts are still of the mixed variety though. In addition, there are quantitative differences as a function of neutron star radius and core temperature. These are discussed in the next section.

\section{Results}

\subsection{Different Regimes of Bursting}

Figure 10 depicts the various bursting regimes as a function of the radius of the neutron star and the accretion rate. The results shown are for core temperatures of $T_{\text {core }}=10^{8} \mathrm{~K}$ and $10^{7} \mathrm{~K}$. The blank areas correspond to stable nuclear burning, the symbols enclosed within circles correspond to prompt bursts and the naked symbols correspond to delayed bursts. The five regimes of bursting behavior described in $\S 4.3$ and summarized in Tables 2,3 are clearly delineated.

Moving from right to left in the panel corresponding to $10^{8} \mathrm{~K}$, we see that at the highest mass accretion rates there is no burst activity since these systems consume hydrogen and helium stably (region I in §4.3). Immediately to the left is a region of delayed mixed bursts (region II). At lower accretion rates, there is a zone of prompt mixed bursts (symbols with circles, region III). Then there is a relatively broad zone of delayed helium bursts (region IV), and finally, at the lowest accretion rates, a region of prompt hydrogen bursts (symbols with circles, region V).

Some of the boundaries between the different zones are remarkably insensitive to the neutron star radius, though the zone of prompt mixed bursts does vary with radius, at least for $T_{\text {core }}=10^{8} \mathrm{~K}$. For very compact neutron stars, e.g., $\log \left(R / R_{S}\right)=0.2$, this zone is reasonably wide, whereas for $\log \left(R / R_{S}\right)=0.6$, the zone almost disappears. The zone of prompt mixed bursts is much broader for $T_{\text {core }}=10^{7} \mathrm{~K}$. These variations with radius and 
$T_{\text {core }}$ lead to noticeable signatures in many of the diagnostics that we discuss later.

In Fig. 10, the points represented with stars correspond to overstable modes with complex $\gamma$ and the triangles correspond to unstable modes with real $\gamma$. In accordance with the discussion in $§ 4.3$, we see that delayed bursts are nearly always overstable, while prompt bursts are either overstable or unstable.

The map of the different burst regimes is roughly the same at other core temperatures, though the widths and locations of the various zones tend to vary. Generally, the regime of delayed mixed bursts becomes somewhat narrower with decreasing $T_{\text {core }}$. Also, for $T_{\text {core }}=$ $10^{8.5} \mathrm{~K}$, there are no hydrogen bursts, i.e., the leftmost region of filled triangles within circles is absent. These patterns may be noticed in several of the plots discussed later.

\section{2. $\quad$ Burst Recurrence Time}

We begin our overview of the observable properties of thermonuclear bursts on neutron stars with the recurrence time $t_{\text {rec }}$, i.e., the average time between bursts. Because we only treat the physics of the accreted layer until a burst is initiated, but not the burst itself, we need to make some assumption as to how much of the available fuel is consumed in a burst. In our calculations we assume that all the fuel is burned, which means that our estimates of $t_{\text {rec }}$ are really upper limits to the observed recurrence time. With this approximation, if $\Sigma_{\text {layer,crit }}$ is the critical column depth of the accreted layer at which a burst is initiated, then the recurrence time $t_{\text {rec }}$ measured by a distant observer is given by

$$
t_{\mathrm{rec}}=(1+z) \frac{\sum_{\text {layer,crit }}}{\dot{\Sigma}} \text {. }
$$

Figure 11 shows $t_{\text {rec }}$ as a function of the accretion luminosity for three choices of the neutron star radius, $\log \left(R / R_{S}\right)=0.2,0.4,0.6$ (i.e., $R=16.4,10.4,6.5 \mathrm{~km}$ ), and four core temperatures, $T_{\text {core }}=10^{8.5}, 10^{8}, 10^{7.5}, 10^{7} \mathrm{~K}$. The $10^{8} \mathrm{~K}$ panel, and to some extent the $10^{7.5}$ $\mathrm{K}$ panel, clearly show the different regimes of bursting behavior discussed in the previous subsection, especially for smaller neutron star radii. The recurrence time tends to become larger for delayed bursts compared to prompt bursts. The straight band of points between $\log \left(l_{\text {acc }}\right) \sim-1$ and -1.6 for the two smaller radii corresponds to prompt mixed bursts. This band is missing for the largest radius because, as seen in Fig. 10, the region of prompt mixed bursts disappears for that radius.

One obvious pattern in Fig. 11 is that there is a general overall increase of $t_{\text {rec }}$ with decreasing $L_{\text {acc }}$. This is because $\dot{\Sigma}$ becomes lower with decreasing accretion rate, so it takes longer to build up a given accretion column. To illustrate this point, Fig. 12 shows the 


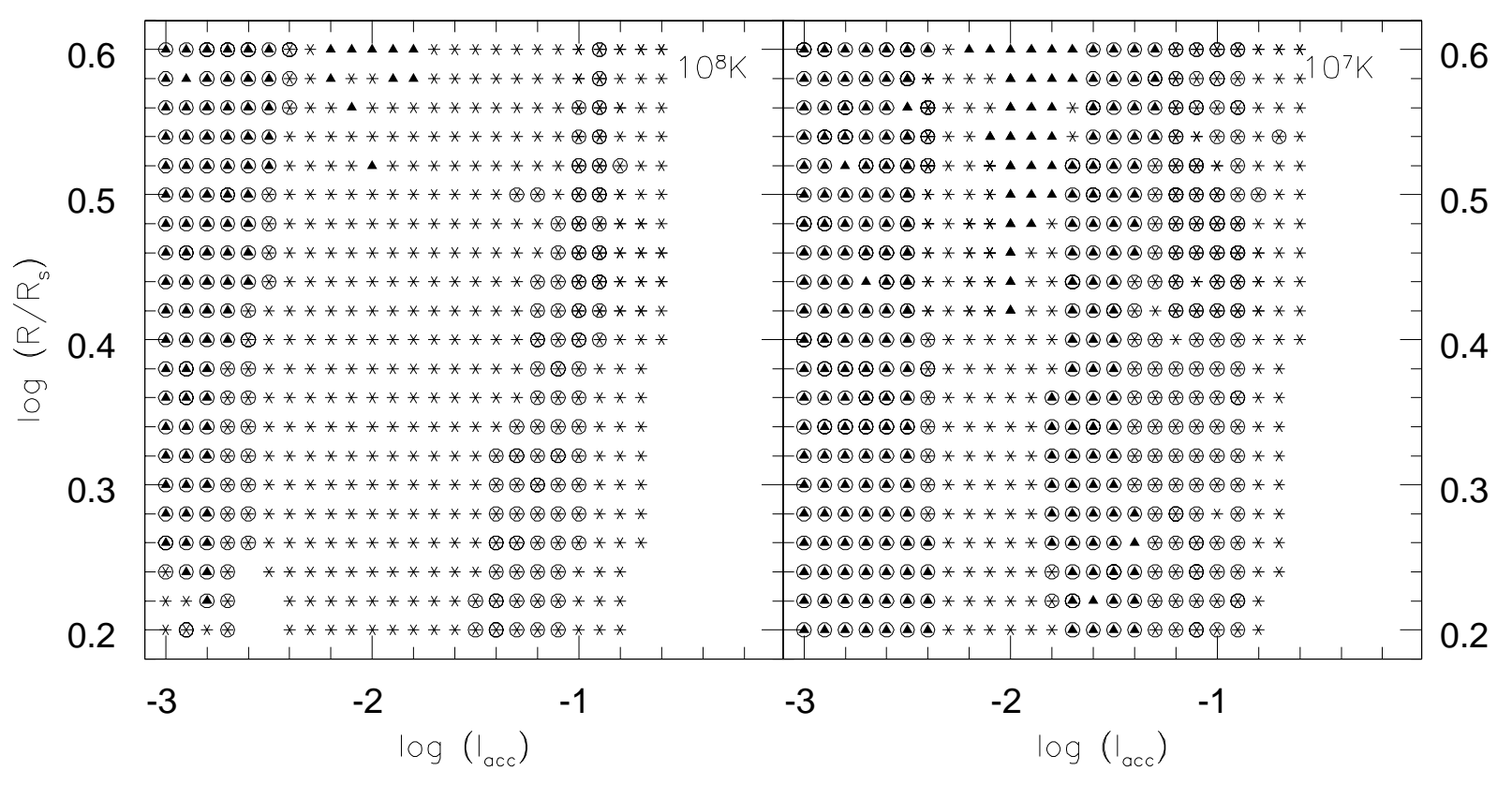

Fig. 10.- Regimes of burning on the surface of a $1.4 M_{\odot}$ neutron star for $T_{\text {core }}=10^{8} \mathrm{~K}$ and $10^{7} \mathrm{~K}$. The empty areas correspond to stable burning, the filled triangles denote regions with unstable modes (real $\gamma$ ), and the stars to regions with overstable/oscillatory modes (complex $\gamma$ ). Prompt bursts (see the text for an explanation) are shown by symbols circumscribed with a circle, and delayed bursts are shown by symbols without circles. Starting from the right, with decreasing luminosity, one goes through the following sequence: stable, delayed mixed bursts, prompt mixed bursts, helium bursts, hydrogen bursts (compare with Table 3). 


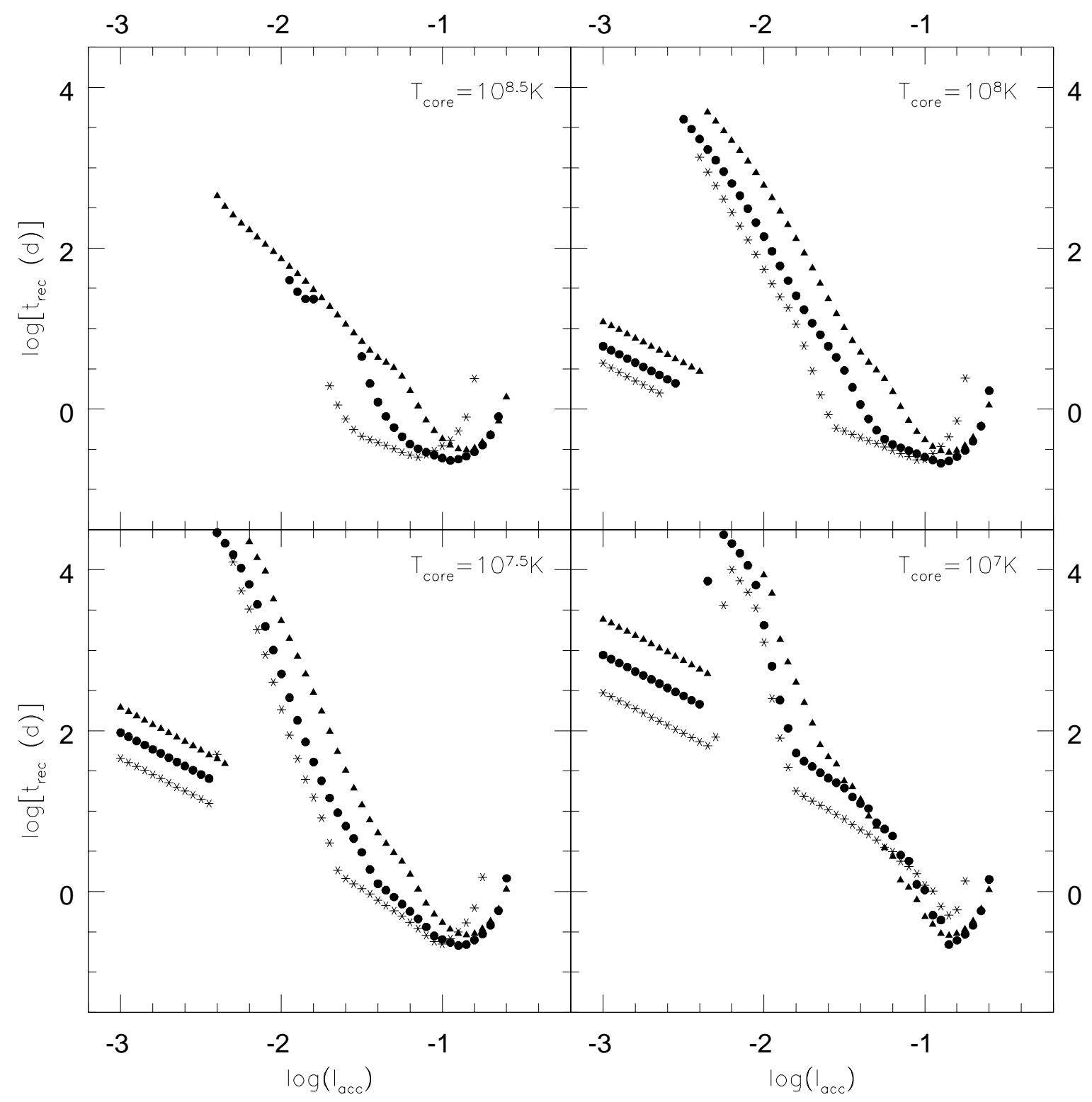

Fig. 11. - The burst recurrence time $t_{\text {rec }}$ for a distant observer, assuming that all the fuel is consumed. The four panels correspond to four core temperatures, and the triangles, circles and stars correspond to neutron star radii of $16.4 \mathrm{~km}, 10.4 \mathrm{~km}$ and $6.5 \mathrm{~km}$, respectively. The four regimes of bursting described in $§ 4.3$ and summarized in Tables 2,3 are clearly seen in many of the sequences. 


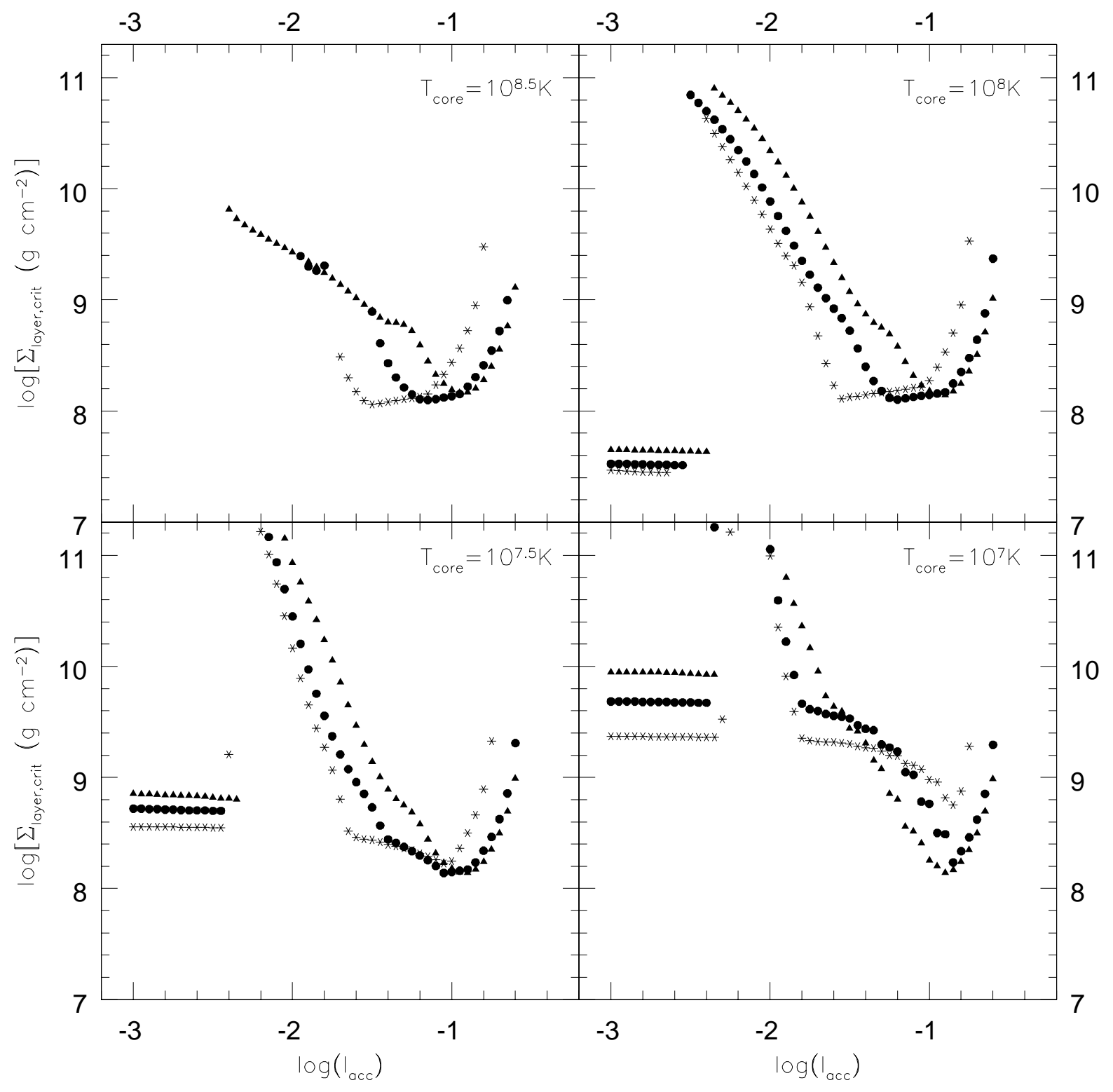

Fig. 12.- Critical column density of accreted material $\Sigma_{\text {layer,crit }}$ at the onset of the burst. The symbols and layout are the same as in Fig. 11. 
dependence of $\Sigma_{\text {layer,crit }}$ for the various cases. We see that, for the prompt mixed bursts, $\Sigma_{\text {layer,crit }}$ is more or less constant.

The results for the other core temperatures are generally reasonable. For $T_{\text {core }}=10^{8.5} \mathrm{~K}$, the recurrence times are fairly similar to the $10^{8} \mathrm{~K}$ case, except that there are no hydrogen bursts at low mass accretion rates. The case of $T_{\text {core }}=10^{7.5} \mathrm{~K}$ is very similar to $10^{8} \mathrm{~K}$. However, for $T_{\text {core }}=10^{7} \mathrm{~K}$, the column needed to produce bursts tends to be higher. This leads to significantly longer recurrence times.

The shortest recurrence time we find in the model is about 5 hours, which occurs at $\log \left(l_{\text {acc }}\right) \sim-1$ for intermediate core temperatures $T_{\text {core }} \sim 10^{7.5}-10^{8} \mathrm{~K}$. The corresponding maximum burst rate is about 5 bursts/day.

\subsection{Burst Fluence}

Probably the most easily observed property of bursts is the total fluence, $E_{\mathrm{H}}+E_{\mathrm{He}}$, resulting from burning the fuel in the accreted layer. We assume that all of the unburnt hydrogen and helium in the accreted layer is fully burned to iron in the burst and radiated during the burst (neglecting any flux going into the star or any energy loss through neutrino emission). We then divide the burst fluence by the Eddington luminosity to calculate an effective burst duration:

$$
t_{\mathrm{H}+\mathrm{He}}=\frac{E_{\mathrm{H}}+E_{\mathrm{He}}}{L_{\mathrm{Edd}}} .
$$

This quantity is plotted in Fig. 13 for our standard three neutron star radii and four core temperatures. As in the case of the recurrence times discussed above, the calculated values are upper limits because of the assumption of complete burning of the fuel.

Since hydrogen burning is typically beta-limited during the burst, the hydrogen fluence may in some cases emerge as a long plateau following the initial spike of helium burning (which is not beta-limited). Therefore, another possibly useful measure of burst duration is the effective duration $t_{\mathrm{He}}$ of helium burning alone, calculated again as fluence divided by the Eddington luminosity. This quantity is plotted in Fig. 14.

Consider first the regime of helium bursts, which corresponds to the segments of $t_{\mathrm{H}+\mathrm{He}}$ and $t_{\mathrm{He}}$ with steep negative slopes in the range $\log \left(l_{\text {acc }}\right) \sim-1.5$ to -2.5 . These are delayed bursts in which the hydrogen burns stably at a shallow depth, while the helium accumulates over a fairly large column before the system goes unstable. The bursts are very much dominated by helium burning (see Fig. 5 ), and so $t_{\mathrm{H}+\mathrm{He}}$ and $t_{\mathrm{He}}$ are nearly equal. Also, the fluence is directly proportional to the accretion column $\Sigma_{\text {layer }}$, as can be seen by comparison 


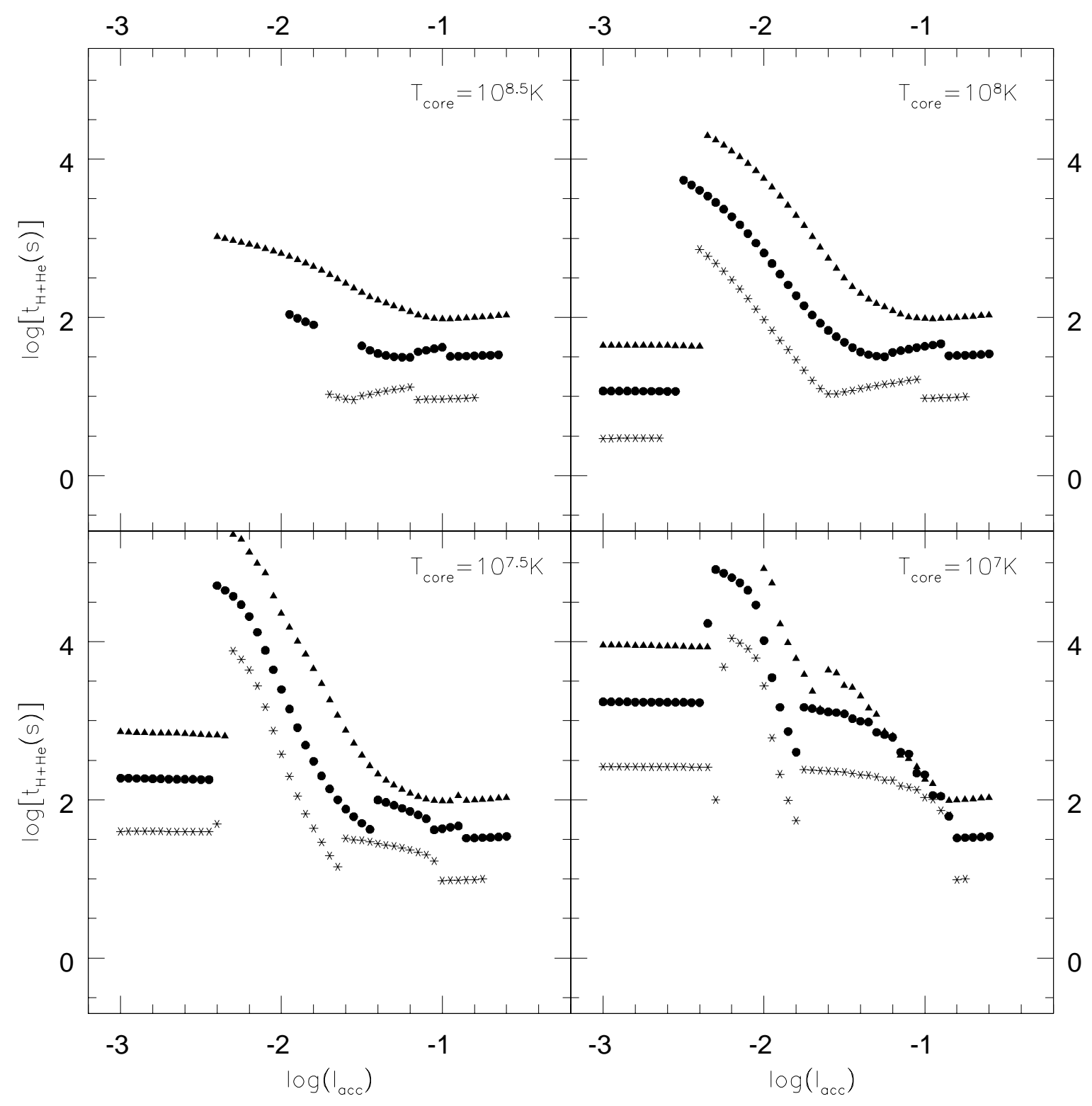

Fig. 13.- Effective burst duration, calculated by dividing the total burst fluence by the Eddington luminosity. Triangles, circles and stars correspond to neutron star radii of $16.4 \mathrm{~km}$, $10.4 \mathrm{~km}$ and $6.5 \mathrm{~km}$ respectively. Notice that, at the extreme right of the various panels, where we have the regime of delayed mixed bursts, the burst duration is either level or falls relative to the prompt mixed bursts, even though the recurrence time $t_{\text {rec }}$ (Fig. 11) and the layer thickness $\Sigma_{\text {layer,crit }}$ (Fig. 12) increase substantially. This indicates that there is considerable steady burning of nuclear fuel in this regime. 
with Fig. 12.

There are two segments of prompt bursts, one at higher luminosities and the other at lower luminosities, on either side of the helium bursts. Both involve significant unburnt hydrogen and helium, and therefore for both $t_{\mathrm{H}+\mathrm{He}}$ is significantly greater than $t_{\mathrm{He}}$ (since hydrogen burning releases more energy per gram, by a factor of about 5, compared to helium burning). Both burst durations are roughly proportional to $\Sigma_{\text {layer }}$ as can be seen by comparison with Fig. 12.

Finally, the delayed mixed bursts, which are present for $\log \left(l_{\mathrm{acc}}\right)>-1$, show a different behavior. These are systems in which considerable stable burning of nuclear fuel occurs in the accreted layer before the layer actually bursts. Therefore, the amount of nuclear fuel available to power the burst is much less than one might expect for the given column depth. The burst durations are at most comparable to, and are often less than, the durations of adjacent prompt bursts, even though these delayed bursts have larger values of $\Sigma_{\text {layer }}$ and correspondingly much longer recurrence times (compare Figs. 13, 14 with Fig. 12).

From Figs. 13, 14 we see that the typical burst durations are predicted to be a few seconds to few tens of seconds at high mass accretion rates. However, the duration can be quite large, an hour or longer, for helium bursts with $\log \left(l_{\text {acc }}\right) \lesssim-2$, and as much as a day for cooler core temperatures.

\subsection{Burst $\alpha$}

The recurrence times and burst durations discussed in the previous subsections were calculated assuming that the entire fuel reservoir is consumed in a burst. However, this assumption may not be valid (e.g. Taam 1982; Fujimoto et al. 1987b), especially for cold neutron stars (Taam et al. 1993). It is, therefore, useful to consider the ratio $\alpha$ of the nuclearburning energy that is emitted during the burst to the total energy released between bursts by accretion. In terms of quantities we have introduced earlier, $\alpha$ is given by

$$
\alpha \equiv \frac{t_{\mathrm{rec}} L_{\mathrm{acc}}}{E_{\mathrm{H}}+E_{\mathrm{He}}}=\frac{t_{\mathrm{rec}}}{t_{\mathrm{H}+\mathrm{He}}} l_{\mathrm{acc}} .
$$

The quantity $\alpha$ should be independent of what fraction of the fuel is burned in bursts; if only a fraction of the fuel is burned, then both $t_{\mathrm{rec}}$ and $t_{\mathrm{H}+\mathrm{He}}$ will decrease by the same fraction and $\alpha$ ought to remain the same. This makes it a particularly useful parameter.

Fig. 15 shows $\alpha$ from our models for several neutron star radii and core temperatures, and a range of accretion rates. Two trends are obvious. First, more compact neutron stars have larger values of $\alpha$ compared to less compact stars; the former release more gravitational 


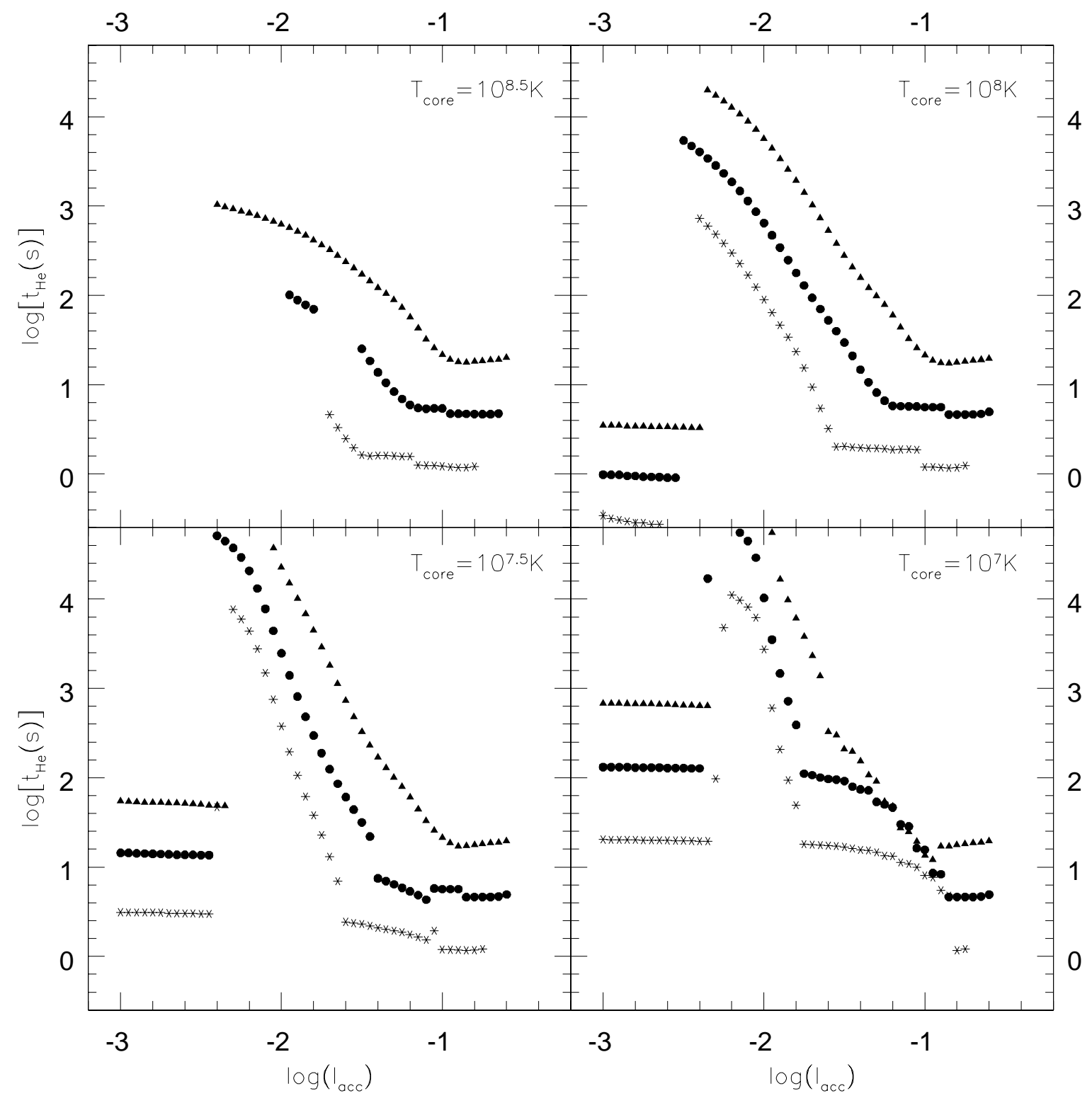

Fig. 14.- Similar to Fig. 13, but for only helium burning. 
energy per unit accreted mass compared to the latter, whereas both release roughly the same amount of nuclear energy in bursts. Second, prompt bursts tend to have smaller values of $\alpha$ compared to delayed bursts. Generally, prompt bursts have a lot of unburnt hydrogen available. This gives a larger burst fluence per unit mass and thus a smaller $\alpha$. The delayed helium bursts have very little hydrogen. Since helium burning releases a factor of about 5 less energy per gram, the $\alpha$ of these bursts is larger by about this factor. The increase of $\alpha$ for the delayed mixed bursts at $\log \left(l_{\text {acc }}\right)>-1$ is for a different reason. Here, the accreting layer has both hydrogen and helium, but both fuels are burned substantially before the burst is initiated. This is the reason for the upturn of $\alpha$ at the highest accretion rates in all the panels in Fig. 15. This trend has not been noted in previous work.

\subsection{Constraints on Neutrino Cooling}

If we assume that the neutron star is in thermal equilibrium as it accretes we may estimate the temperature of its core as a function of its mean accretion rate. There are two contributions to energy flowing into the core. The first is the inward flux at the inner boundary of the accreted layer and the substrate; this may be either positive or negative (see Brown 2000). The second is the energy of released by nuclear reactions deep in the crust (Brown et al. 1998), typically $\sim 1 \mathrm{MeV} /$ baryon. Our calculations show that the former contribution is much smaller than the latter; this is consistent with the discussion in Cumming \& Bildsten (2000) where the authors estimate that, out of the $1 \mathrm{MeV}$ per baryon released by deep crustal nuclear reactions, only about $150 \mathrm{keV}$ per baryon escapes to the surface. Therefore, for an approximate estimate of the core temperature, it is reasonable to assume that the flux flowing into the core is equal to $1 \mathrm{MeV} /$ baryon (see Brown 2000 for a more detailed discussion including the effect of different assumptions on the thermal conductivity). If we further assume the simple estimates of the neutrino emissivity given in Shapiro \& Teukolsky (1983), we may estimate the equilibrium temperature of the core for a given accretion rate. For the modified URCA process we find

$$
\left(\frac{T_{\text {core }}}{10^{8} \mathrm{~K}}\right)^{8}=2500 \frac{E_{\mathrm{DH}}}{1 \mathrm{MeV} / \text { baryon }} \frac{(1+z)^{2}}{z} \frac{\dot{M}}{\dot{M}_{\text {Edd }}}\left(\frac{\rho}{\rho_{\text {nuc }}}\right)^{1 / 3},
$$

which gives a typical temperature in the range $(1-3) \times 10^{8} \mathrm{~K}$. If the neutron star cools via the direct URCA process from (say) a pion core, then the core temperature is

$$
\left(\frac{T_{\text {core }}}{10^{7} \mathrm{~K}}\right)^{6}=9 \frac{E_{\mathrm{DH}}}{1 \mathrm{MeV} / \text { baryon }} \frac{(1+z)^{2}}{z} \frac{\dot{M}}{\dot{M}_{\mathrm{Edd}}} \frac{\rho}{\rho_{\text {nuc }}} \theta^{-2},
$$

where $\theta \sim 0.3$. In this case, the core is significantly cooler, $T_{\text {core }} \sim 10^{7} \mathrm{~K}$. In both cases, the core temperature depends only weakly on the mean accretion rate (as $\dot{M}^{1 / 8}$ and $\dot{M}^{1 / 6}$, 


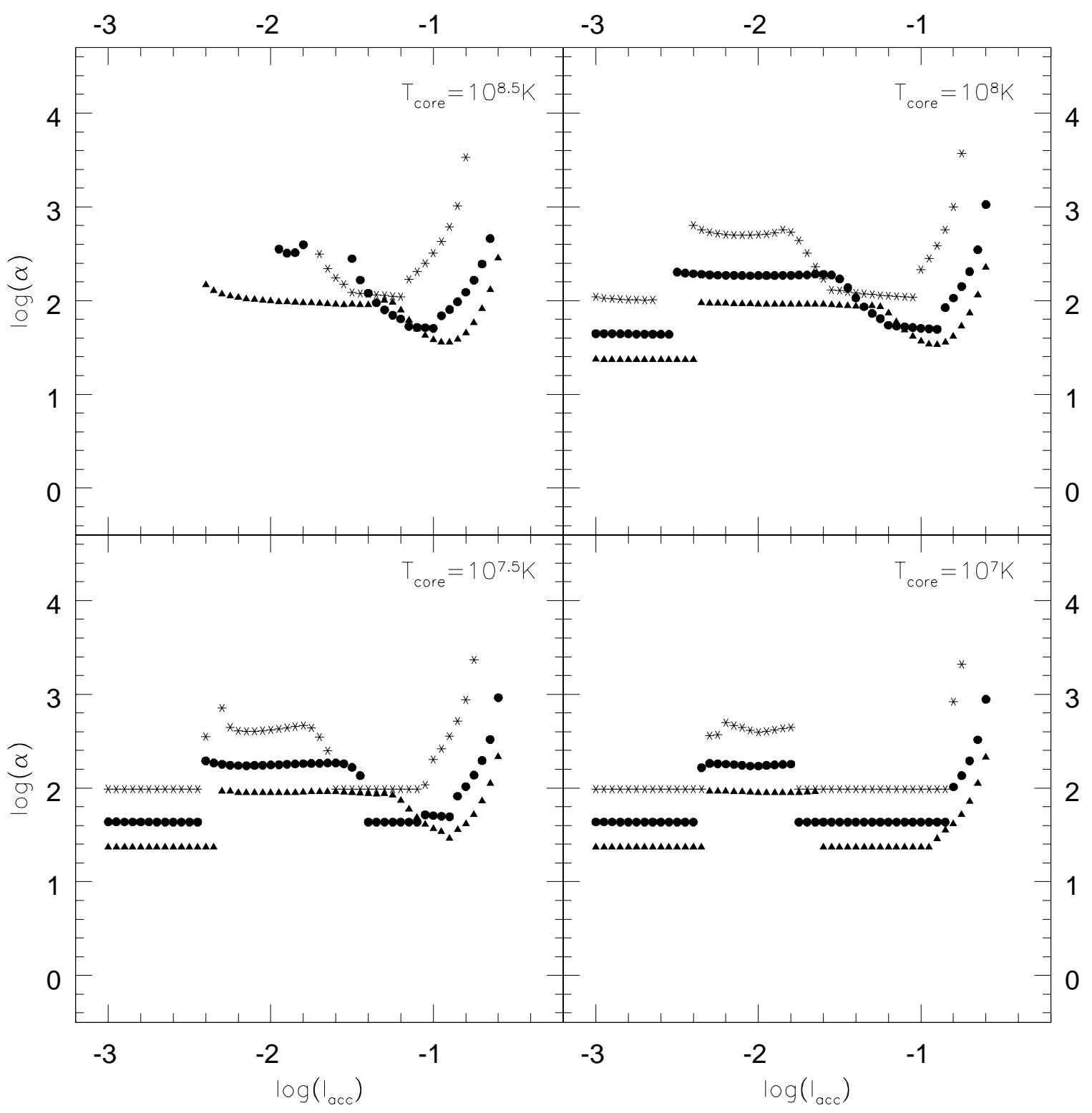

Fig. 15.- Dependence of the parameter $\alpha$, the ratio of the energy generated by accretion between bursts to the nuclear energy emitted during bursts. Triangles, circles and stars correspond to neutron star radii of $16.4 \mathrm{~km}, 10.4 \mathrm{~km}$ and $6.5 \mathrm{~km}$, respectively. Notice the steep rise of $\alpha$ at high accretion luminosities, near the stable burning limit. The rise is characteristic of delayed mixed bursts. 
respectively) and on the exact flux flowing into the core (whether it is $1 \mathrm{MeV}$ per baryon or a little more or less.)

An important caveat is that our results for cool neutron stars with $T_{\text {core }} \sim 10^{7} \mathrm{~K}$ depend somewhat sensitively on how we treat the inner boundary condition (see $\S 2.2$ ), so the bursting behavior of stars with direct URCA cooling may not be precisely as depicted by our results for $10^{7} \mathrm{~K}$; however, it should be noticeably different from that of stars with modified URCA cooling.

\section{Discussion}

We begin in $\S 6.1$ with a brief summary of how the results depend on the input physics. We follow that in $\S 6.2$ with a discussion of different kinds of modes present in our model and use this to explain the occurrence of delayed mixed bursts. In $\S 6.3$, we discuss how our methods compare with the many previous theoretical analyses of Type I bursts in neutron stars. We then present in $\S 6.4$ a preliminary comparison of our theoretical predictions with observations, making extensive use of the review of EXOSAT data published by van Paradijs et al. (1988). We defer a detailed comparision with more recent observations with the Rossi X-ray Timing Explorer and BeppoSAX to a subsequent paper, but briefly touch on the work of Cornelisse et al. (2003). (The latter paper was posted after our paper was submitted

to the journal. Since their results are very relevant for this work, we have included some discussion.) Finally, in $\S 6.5$, we discuss some improvements to this work that may be worth pursuing.

\subsection{Sensitivity to Input Physics}

In the course of doing this work, we changed several prescriptions for the input physics, usually starting with a simple approximation and graduating later to more realistic prescriptions. By monitoring how the results changed we have developed a sense of which aspects of the input physics are most important if one is interested in accurate results.

In the equation of state, the only complication is the electron pressure. Before settling on the quadrature prescription given in equation (22), which is taken from Paczynski (1983b), we tried a simpler prescription in which we wrote the electron pressure as the straight sum of $P_{\mathrm{e}, \mathrm{nd}}$ and $P_{\mathrm{e}, \mathrm{d}}$. The differences in the results are insignificant. Similarly, we tried modeling the nuclear reactions both with and without screening. There was little difference, except perhaps at the lowest accretion rates, $\log \left(l_{\text {acc }}\right) \sim-3$, where the hydrogen bursts showed 
some small variations.

The effect of changing the opacity prescriptions is more serious. For instance, the opacities of Hernquist \& Applegate (1984), which we tried first, are generally lower than those of Potekhin (1999). Therefore, with the former, a thicker layer must accumulate before the layer becomes unstable. This effect is strongest for the hydrogen bursts, and yields longer recurrence times and larger fluences. Also, because the burning layer is more poorly insulated from the core, the results tend to be more sensitive to the core temperature than when we use the modern conductive opacities of Potekhin (1999). As a result, with the Hernquist \& Applegate (1984) opacities we find that models with $T_{\text {core }}=10^{7.5} \mathrm{~K}$ are more similar to models with $T_{\text {core }}=10^{7} \mathrm{~K}$, whereas with the Potekhin (1999) opacities, they are more similar to $10^{8} \mathrm{~K}$.

The results are even more sensitive to the radiative opacity prescription. Prior to settling on the Schatz et al. (1999) prescription, we employed the simple fitting function of Iben (1975). The results were qualitatively the same as the ones presented here, except that the boundaries between the different regimes moved towards lower values of $\log \left(l_{\mathrm{acc}}\right)$ by about 0.2. That is, the patterns in Figs. 5, 10-15 were shifted to the left by this amount, but not altered very much in shape. In view of this, it may be worthwhile to incorporate an even better approximation for the radiative opacity in future calcualtions.

The frequencies and structure of the unstable modes are not changed significantly by the opacity with the exception that the modes penetrate deeper into the substrate for lower opacities.

Finally, we have found that the inner boundary condition plays an important role. Figure 16(a) shows two sets of calculations, both of which set the temperature equal to $T_{\text {core }}$ at the inner boundary. In one case, the matching is done inside the substrate at a depth $\Sigma_{\text {diff }}$ determined by a diffusion criterion (see $\$ 2.2$ and eq. 18 for details), while in the other the boundary condition is set at $\Sigma_{\text {layer }}$, the bottom of the accreted layer. There is an enormous difference in the results. In the former case, which is more physical, the temperature of the accreted layer tends to remain high and the temperature profile relaxes to $T_{\text {core }}$ only well inside the star. In the latter case, however, the boundary condition forces the gas layer to be cool near the bottom and this causes the rest of the layer to be cooler than in the previous case. The lower temperature delays the onset of nuclear burning, causing the critical surface density for instability to go up significantly.

We feel that we have captured a good fraction of the important physics with the boundary condition that we use. Nevertheless, there is probably room for further improvement. 

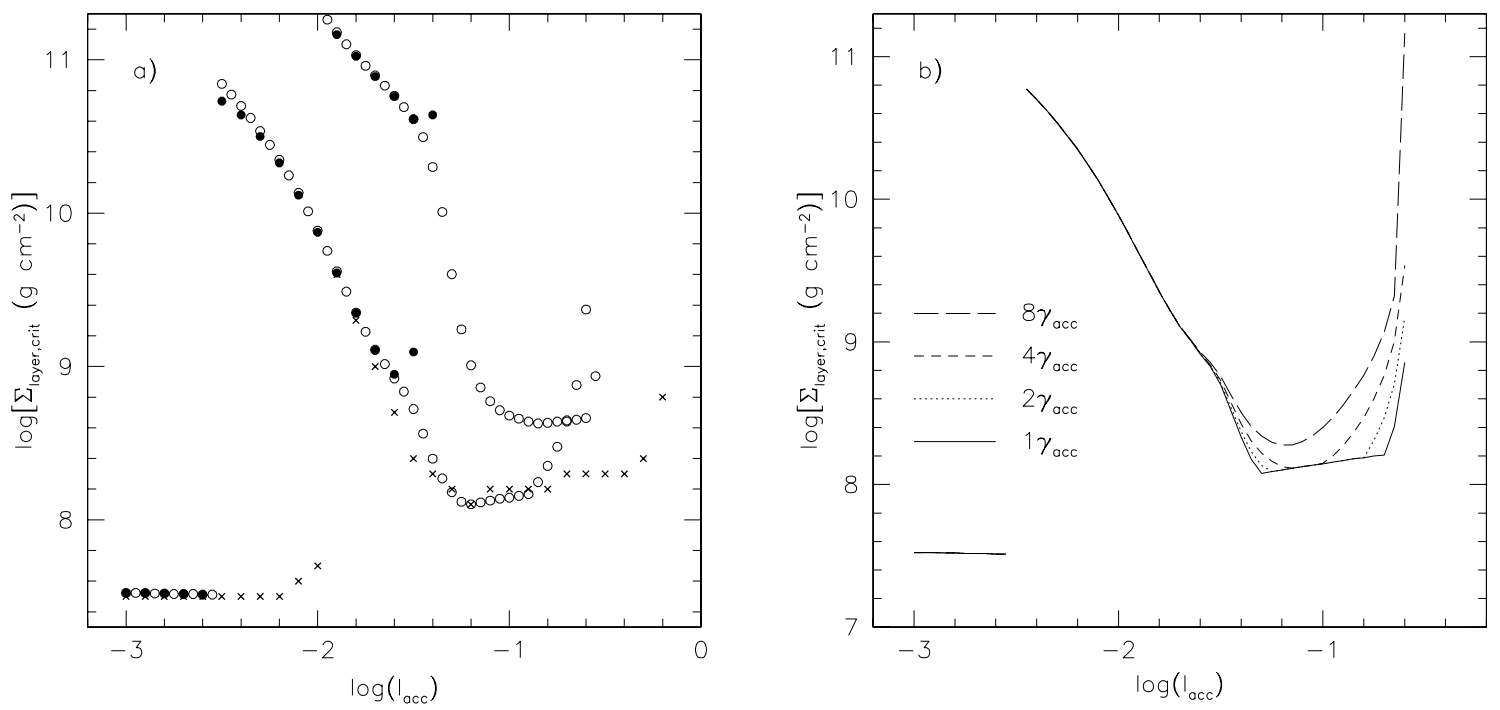

Fig. 16. - Results for $T_{\text {core }}=10^{8} \mathrm{~K}, R=10.4 \mathrm{~km}, M=1.4 \mathrm{M}_{\odot}$. (a) Shows how the restriction to thermal modes (filled circles) affects the results. Compared to the full instability calculation (open circles), only a fraction of the range of $l_{\text {acc }}$ is found to be unstable for purely thermal perturbations. The results depicted by crosses use the instability criterion of Fushiki \& Lamb (1987a). The lower set of circles corresponds to our standard boundary condition in which the temperature boundary condition is applied inside the star at a diffusion depth. The upper set of circles depicts the results if the temperature boundary condition is applied at the bottom of the fuel layer [as in Narayan \& Heyl (2002) and Fushiki \& Lamb (1987a)]. (b) Shows how the choice of $g_{\text {mode }}$ affects the onset of instability. The larger the value of $g_{\text {mode }}$, the more the nuclear modes near the right end of the plot are delayed. The thermal modes on the left are hardly affected. 


\subsection{Thermal Modes, Nuclear Modes, and Delayed Mixed Bursts}

Because the eigenmodes and growth rates are calculated by perturbing five coupled differential equations, the physics of the modes is not always apparent. We make a beginning here by sorting the modes into two major classes.

To understand the division, we note that the perturbation equations written in Appendix A involve three time derivatives, one each in the energy equation (A10), the H-evolution equation (A6) and the He-evolution equation (A12). The characteristic time scale of the energy equation is something like the diffusion time, which is usually quite short, whereas the characteristic time scale of the other two equations is the accretion time, since this is the time on which the composition of a parcel of gas changes. We thus expect fast-growing modes to mostly involve the the energy equation (plus the equations of hydrostatic equilibrium and energy transfer which do not involve time derivatives), while slower modes should involve significant perturbations in all variables, including $X$ and $Y$. We refer to the former as "thermal modes" and the latter as "nuclear modes."

It is straightforward to filter out the nuclear modes in the calculations - we just switch off perturbations in $X$ and $Y$ by setting $X(\Sigma)=X_{0}(\Sigma), Y(\Sigma)=Y_{0}(\Sigma)$, or equivalently, $X_{1}(\Sigma)=Y_{1}(\Sigma)=0$. Figure 16(a) shows the results of such a calculation. Compared to our standard calculation in which all variables are allowed to vary (open circles), we see that thermal modes (filled circles) are present only for lower accretion rates, $\log \left(l_{\text {acc }}\right)<-1.5$, i.e., in the regime of helium bursts and hydrogen bursts, but not at higher accretion rates where mixed bursts occur. An inspection of the mode growth rates confirms that these modes grow on relatively short time scales; typically, the growth time for thermal modes is a factor of tens to hundreds shorter than the accretion time scale. The modes above $\log \left(l_{\text {acc }}\right) \sim-1.5$, which are present only when all variables are allowed to vary, are the nuclear modes in our classification. These modes have slow time scales, and their eigenfunctions involve important fluctuations in composition, which is not the case with the thermal modes. The eigenfunction shown in Figs. 8, 9 is a nuclear mode.

The identification of the slow nuclear modes provides a natural explanation for the category of delayed mixed bursts that we have newly identified in this paper. Recall that we do not consider a system to be unstable unless the growth rate $\Re(\gamma)$ is greater than $g_{\text {mode }} \gamma_{\text {acc }}$ (see eq. 21). For our nominal choice of $g_{\text {mode }}=3$, the thermal modes are not in the least affected by the value of $g_{\text {mode }}$, since their growth rates are typically much greater than the limit. However, the slower nuclear modes are strongly influenced by the criterion, and the effect is particularly severe at higher accretion rates. The right panel of Figure 16 shows the critical column for bursts $\Sigma_{\text {layer,crit }}$ as a function of $\log \left(l_{\text {acc }}\right)$ for different choices of $g_{\text {mode }}$. The delayed burst regime becomes more and more prominent as $g_{\text {mode }}$ increases. The calculations 
show that, for high accretion rates, the accreted layer is marginally unstable already at small values of $\Sigma_{\text {layer }}$. However, the growth rate is low, and the instability takes a while to grow. By the time the mode has grown enough to produce a burst, $\Sigma_{\text {layer }}$ is significantly larger; this is the reason for the delay in the burst. In addition, because of the delay, much of the fuel is burned stably prior to the burst, and the amount of unburnt fuel available for the burst is reduced. This causes a dramatic increase of $\alpha$ in this regime. The extent of these effects depends on the choice of $g_{\text {mode }}$. The value we have used for the results presented in $\S 5, g_{\text {mode }}=3$, are in our opinion reasonable.

\subsection{Comparison with Earlier Theoretical Work}

Previous investigations have identified the various burning regimes that we have found here (Fujimoto et al. 1981; Fushiki \& Lamb 1987a; Bildsten 2000), with the exception of the regime of delayed mixed bursts (see $\S 4.3$, Tables 2,3 , Fig. 10). The ranges of $l_{\text {acc }}$ of the different regimes obtained by these workers generally agree with our results, though we have found several new features, as discussed in previous sections. Our method, being more rigorous, promises to provide better quantitative predictions for comparison with observations. Also, we calculate mode frequencies, which enables us to identify overstable modes and to estimate the oscillation periods. It is unclear if this will have clear observational signatures, but it is a topic worthy of further investigation.

As described in $\S 1$, our method is similar in spirit to previous investigations that evaluated steady-state configurations and then studied the stability of the equilibria to small perturbations (for example Hansen \& van Horn 1975; Ergma \& Tutukov 1980; Fujimoto et al. 1981; Fushiki \& Lamb 1987a; Cumming \& Bildsten 2000). The computation of the equilibria in the various studies probably do not differ a great deal since the physics is basically well understood ( $(2)$. The approximations that we and others use (§2.3) appear to be harmless, except perhaps the opacity which does make a difference (§6.1). Once one has calculated a sequence of equilibria such as those shown in Figs. 2, 3, it is necessary to determine at what $\Sigma_{\text {layer,crit }}$, if any, the accreted layer becomes unstable. It is at this stage that the major differences in methods appears.

The technique that we have used in this paper is new and avoids any prejudices as to which processes are important and which are not for triggering a burst. We carry out a full linear stability analysis of the equilibrium solution by solving for the eigenmodes and their complex eigenfrequencies. In particular, the regime of delayed mixed bursts that we have found ( $\$ 4.3$, Tables 2,3$)$, and the identification of nuclear modes $(\S 6.2)$, are entirely the result of the more rigorous formalism we employ. As we shall see below, the delayed mixed burst 
regime may help to explain some puzzling observations. Also, none of the previous studies was able to identify whether the growing mode is a simple instability or an overstability. This too may have some observational implications.

Earlier works have generally used various local or approximately global criteria, calculated at or near the bottom of the layer, to determine the stability of the accreted layer. With the exception of Fushiki \& Lamb (1987a), no one to our knowledge has discussed global perturbations of the steady-state configuration. Even the Fushiki \& Lamb (1987a) study was relatively crude since it assumed a constant temperature perturbation as a function of depth and did not treat perturbations in the substrate below the layer. In contrast, our approach involves a computation of the entire eigenfunction from first principles, without any preconceptions as to the shape of the mode, and allows for perturbations in the substrate. It should be emphasized that we consider perturbations in all variables, including the composition parameters $X, Y$ and $Z(=1-X-Y)$. This enables us to find both thermal modes and nuclear modes $(\S 6.2)$. In contrast, all previous studies restricted themselves to thermal perturbations, that too approximately, and thus were sensitive only to thermal modes.

To get some sense of how good the approximate criteria of the past are, we present here some quantitative comparisons with two methods described in the literature, those of Fushiki \& Lamb (1987a) and Cumming \& Bildsten (2000). These papers use two different criteria for the onset of instability. Both express the criterion as

$$
\frac{d \epsilon_{\mathrm{heat}}}{d T}>\frac{d \epsilon_{\mathrm{cool}}}{d T}
$$

but differ in their definitions of the quantities. Fushiki \& Lamb (1987a) define $\epsilon_{\text {heat }}$ to be the total nuclear energy generation rate while Cumming \& Bildsten (2000) define it to be 1.9 times the energy generation rate from the triple-alpha reacton. In order to include the hydrogen bursts in the latter calculation we generalized it by adding also the hydrogen energy generation rate. The differences in the cooling rates in the two approaches are more striking. Fushiki \& Lamb (1987a) asume that $d T_{1} / d \Sigma=0$ and use a formula that is equivalent to (this is our best guess since their paper does not explain in sufficient detail)

$$
\frac{d \epsilon_{\mathrm{cool}}}{d T}=\frac{d}{d T}\left(\frac{d F}{d \Sigma}\right)=\frac{d \ln K}{d T} \epsilon-\frac{d^{2} \ln K}{d T d \Sigma} F,
$$

where $\epsilon$ is the total energy generation rate and $K=16 \sigma T^{3} / 3 \kappa$ is the thermal conductivity (see eq. 4). Cumming \& Bildsten (2000), on the other hand, start from the work of Fujimoto et al. (1981) and write approximately

$$
\frac{d \epsilon_{\mathrm{cool}}}{d T}=\frac{d}{d T}\left(\frac{a c T^{4}}{3 \kappa \Sigma^{2}}\right)
$$


where the quantity on the right is roughly the thermal energy density divided by the diffusion time to the surface. Clearly both criteria are approximate. Both are also local since they focus on values at a particular depth. The Fushiki \& Lamb (1987a) method could be generalized to an approximate global criterion (it is possible that the authors did make such a generalization, but we have not been able to understand exactly how they might have done it). We limit ourselves to the local version given above. The Cumming \& Bildsten (2000) criterion is of a mixed kind, since it compares a local quantity for the heating rate to a pseudo-global quantity for the cooling rate.

We use the equilibria as computed with our code using our particular prescriptions for opacity, equation of state, etc. (§2.3) for all the calculations. We thereby ensure a fair comparison of the different methods. We consider the calculations done with our full linear perturbation analysis as the "correct" answer. Indeed, since the two approximate criteria described above are both restricted to thermal perturbations, we feel that the "correct" answer is the set of thermal modes calculated with our perturbation analysis (filled circles in Figs. 16 and 17).

Figure 16(a) shows results corresponding to a temperature boundary condition, with the crosses depicting the results obtained with the Fushiki \& Lamb (1987a) criterion. For the latter, we applied the local criterion (38), (39) at the temperature maximum in the accreted layer. (By choosing this point rather than the bottom of the layer, we feel that the method has a better chance of approximating the true global problem.) The agreement between the approximate criterion and our full calculations (thermal plus nuclear modes) is quite good. The Fushiki \& Lamb (1987a) criterion finds a smaller range of $l_{\text {acc }}$ for the helium bursts and overestimates the range of the hydrogen bursts. Also, not surprisingly, it does not find the delayed mixed burst regime. The most surprising result is that the method, which is overtly limited to thermal perturbations, finds modes well outside the range of thermal modes. This may be viewed either as a serious error in the method or as an unexpected strength!

Figure 17 shows the corresponding comparison for the Cumming \& Bildsten (2000) criterion. Since these authors use a flux inner boundary condition, we calculated the equilibria as well as our stability results for this boundary condition, assuming an outward flux of 150 $\mathrm{keV}$ per accreted baryon at the bottom of the accretion layer. In the stability analysis we assumed that the perturbed flux is zero at the bottom of the layer (which corresponds to a non-zero temperature perturbation). Once again, we should view the thermal modes (filled circles) as the "correct" answers. The crosses show the range of instability according to the criterion (38), (40) applied at the bottom of the accreted layer (where the temperature reaches a maximum). Wherever the Cumming \& Bildsten (2000) criterion indicates that there is an instability, the agreement in the value of $\Sigma_{\text {layer,crit }}$ is fairly good. However, the 


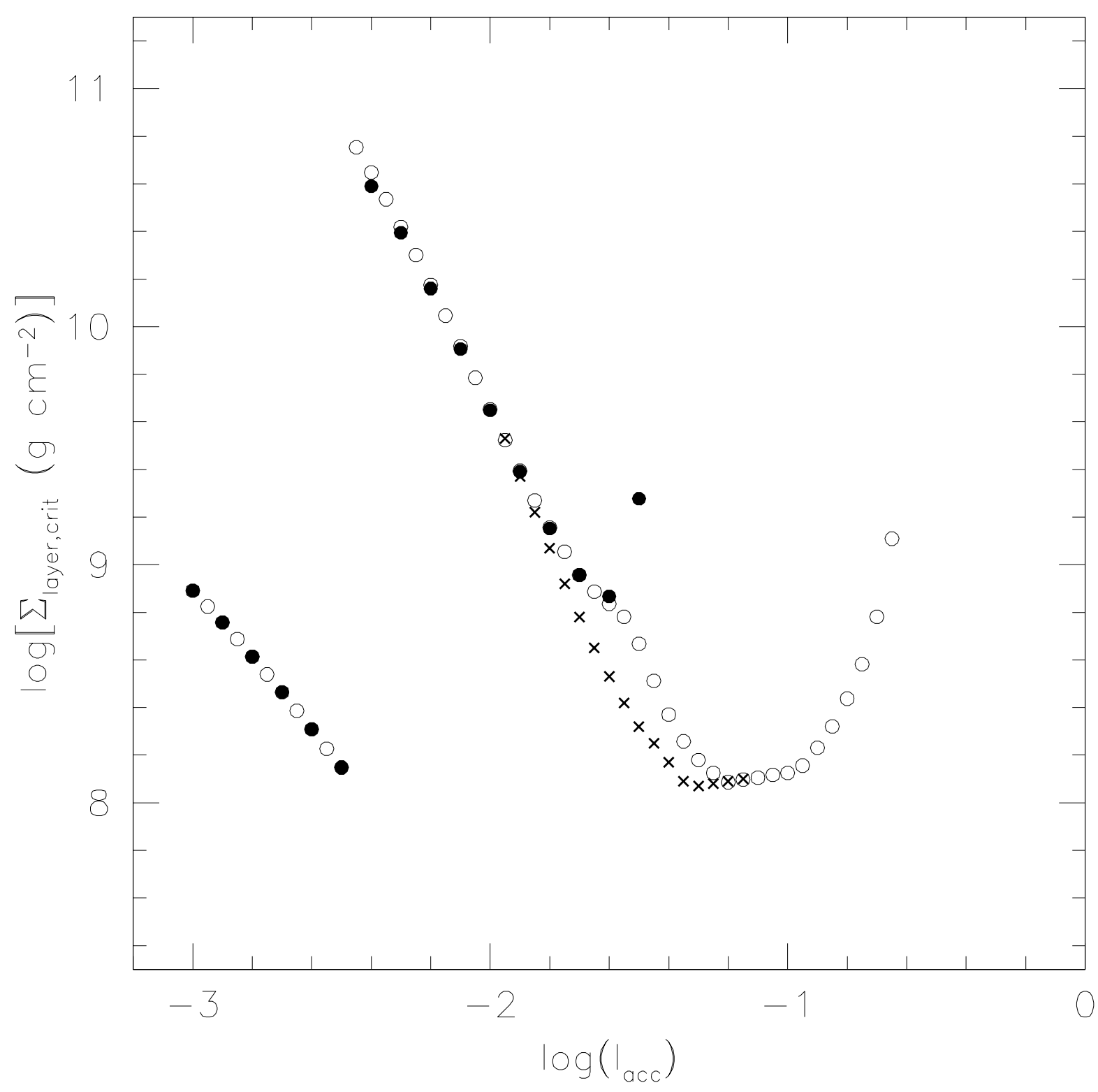

Fig. 17.- Models for $\log R / R_{s}=0.4$ and $M=1.4 \mathrm{M}_{\odot}$ with a flux inner boundary condition. It is assumed that a constant flux of $150 \mathrm{keV}$ per accreted baryon flows out of the star into the accretion layer. The open circles correspond to the full calculation where all variables are perturbed, and the filled circles to a calculation in which the composition is not allowed to vary (thermal modes). The crosses trace the results corresponding to the instability criterion of Cumming \& Bildsten (2000). 
method appears to miss finding an instability over a fairly wide range of $l_{\text {acc }}$. Also, naturally, the method does not reproduce the delayed mixed bursts (which we have identified as an effect associated with nuclear modes).

One comment is in order. In calculating the above results for the Fushiki \& Lamb (1987a) and Cumming \& Bildsten (2000) criteria, we followed the philosophy described in $\S 3$, namely to label a system unstable only if it has unstable configurations on the "wall" of the S-curve for values of $\Sigma_{\text {layer }}$ above the hydrogen and helium peaks. Thus, a situation like Fig. 2(a) in which the wall solutions are all stable is considered stable, even though there are unstable solutions on the trailing slope of the helium peak. We do not know if the other authors used this criterion or not, but we believe it is the correct approach to the problem (for reasons discussed in $\S 3$ ).

In summary, the two approximate criteria discussed above are useful for quick estimates, but are not appropriate for detailed quantitative results. For the latter, one needs to do a full linear stability analysis, as presented in this paper, or carry out time-dependent simulations of the kind reviewed in $\S 1$.

The good news is that, in the regions where both our and previous work have found instability, the results agree quantitatively quite well. Specifically, the results presented by Fujimoto et al. (1987b) agree in detail with the equilibrium curve presented in Fig. 4(a). For low accretion rates and cool cores, Hanawa \& Fujimoto (1986) and Fujimoto et al. (1987a) found very long X-ray bursts with durations similar to those shown in Fig. 13. We also find good qualitative agreement with the more comprehensive results of Fushiki \& Lamb (1987a) and the different burning regimes that they have identified, and quantitative agreement with the tabulated column densities and temperatures in Table 2 of Cumming \& Bildsten (2000). However, the overarching nature of the calculations presented here make it difficult to compare very precisely with earlier work which were generally focused on understanding specific ignition criteria, rather than presenting predictions of the gross properties of Type I bursts over a wide range of accretion rates, temperatures and stellar radii.

\subsection{Comparison with Observations}

\subsubsection{Stable Burning Limit}

Van Paradijs et al. (1988) have collected together a body of burst data obtained with the EXOSAT satellite. They define a quantity $\gamma$ to be the ratio of the observed persistent flux $F_{p}$ to the net peak flux $F_{\text {re }}$, not including the persistent emission, of bursts with radius

expansion. The quantity $F_{p}$ is proportional to $L_{\text {acc }}$, while $F_{\text {re }}$ is proportional to $L_{\text {Edd }}^{\prime}$, where 
$L_{\text {Edd }}^{\prime}$ is the Eddington luminosity in the frame of the neutron star surface and is smaller than our $L_{\text {Edd }}$ (defined at infinity) by a redshift factor. Thus, $\gamma$ is larger than $L_{\text {acc }} / L_{\text {Edd }}$ by a variable factor that may be $\sim 1.5$. Van Paradijs et al. (1988) find that bursts occur for $\log \gamma \lesssim-0.5$; sources with $\gamma$ above this limit apparently burn fuel stably without bursting. The observational limit they have obtained on $\gamma$ should be compared with our prediction that bursts occur only below $\log \left(L_{\text {acc }} / L_{\text {Edd }}\right) \sim-0.7$ to $-0.6(\S 4.3)$. The agreement is fairly good. In a previous paper (Narayan \& Heyl 2002), we found that bursts should occur up to $\log \left(l_{\text {acc }}\right) \sim-0.5$. However, those calculations were done with a less sophisticated inner boundary condition (see $\S 2.2$ ).

The brightest low-mass X-ray binaries generally do not show burst activity. Matsuba et al. (1995) saw Type I bursts from the bright source GX 13+1 when $\gamma \sim 0.3$, Kuulkers \& van der Klis (2000) saw a radius-expansion burst from the source GX 3+1 when the persistent luminosity was $0.17 L_{\mathrm{Edd}}$, and Kuulkers, van der Klis \& van Paradijs (1995) and Smale (1998) observed bursts from Cyg X-2 with $\gamma$ as large as 0.74. Tournear et al. (2003) have recently used the USA satellite to carry out long-duration observations of a number of neutron star systems. They observed bursts from sources with a range of $l_{\text {acc }}$ extending up to $\sim 0.3$. Finally, Cornelisse et al. (2003) find that bursts cease at a luminosity of $5.5 \times 10^{37} \mathrm{erg} \mathrm{s}^{-1}$, which corresponds again to $0.3 L_{\mathrm{Edd}}$ (for $M=1.4 M_{\odot}$ and our definition of the Eddington luminosity). These observations are all generally consistent with the van Paradijs et al. (1988) results. The stable limit according to our model is encouragingly close to the data, which is noteworthy since our model is a first-principles calculation with no free parameters.

\subsubsection{Burst $\alpha$}

As mentioned in $§ 5.4$, both the predicted recurrence time and the fluence of bursts are subject to an uncertainty over whether the entire reservoir of fuel is consumed during the burst. Only fully time-dependent calculations can predict what fraction of the fuel is consumed in the burst, and even then probably only with multi-dimensional simulations. This is well beyond the scope of our calculation. However, partial burning affects the recurrence time and the burst fluence in an identical fashion, and therefore their ratio should be insensitive to this uncertainty. The parameter $\alpha$ defined in $§ 5.4$ is such a quantity.

Van Paradijs et al. (1988) present results for $\alpha$ as a function of $\gamma$. Fig. 18 compares their data with our predictions. In plotting the data, we have assumed that the observed $\gamma$ is equal to $l_{\text {acc }}$. As discussed in $\S 6.4$, the two may differ by a few tens of percent (because of gravitational redshift), so the data may need to be shifted to the left by this amount. Allowing for this uncertainty, we find that the data agree with the model predictions fairly well for 


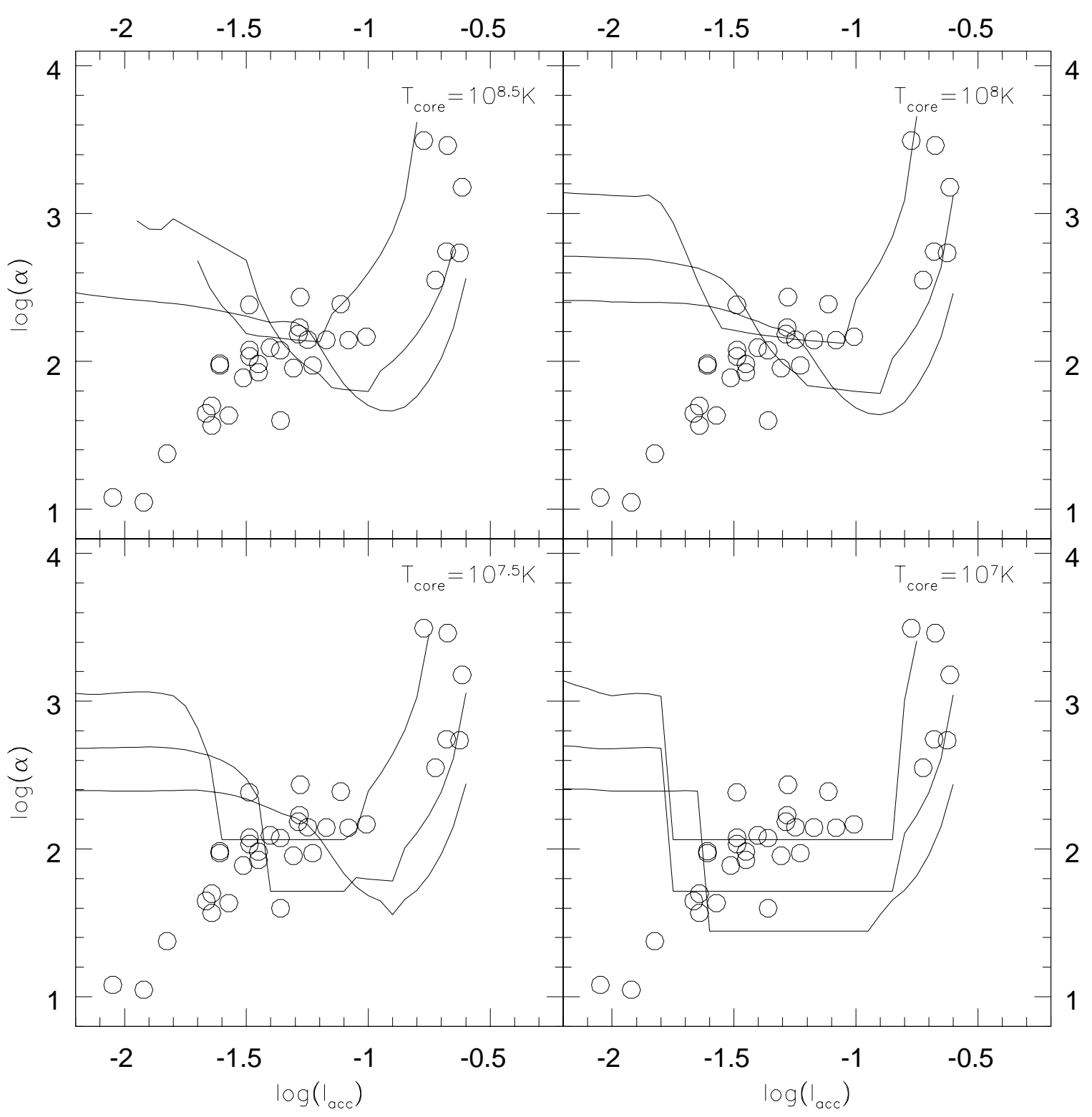

Fig. 18. - A comparison of the model predictions for $\alpha$ with the observational data presented in van Paradijs et al. (1988). The circles show the data. The lines in each panel correspond to the model results shown in Fig. 15, with the symbols replaced by connected lines. In each panel, the uppermost line corresponds to a neutron star radius of $6.5 \mathrm{~km}$, the middle line to $10.4 \mathrm{~km}$, and the lowest line to $16.4 \mathrm{~km}$. 
luminosities above $\log \left(l_{\text {acc }}\right) \sim-1.5$. For accretion luminosities in the range $\log \left(l_{\text {acc }}\right) \sim-1.5$ to -1 , the data give $\alpha \sim 10^{2}$. This value is roughly consistent with our model predictions for smaller neutron star radii $(6.5 \mathrm{~km}$ and $10.4 \mathrm{~km})$; the largest radius we have tried $(16.4$ $\mathrm{km}$ ) does not agree very well with the data.

The most interesting feature of the data is that, for higher accretion luminosities, $\alpha$ shoots up to values $\sim 10^{3}$. Van Paradijs et al. (1988) suggested that the increase is because much of the nuclear energy in the accumulating fuel is released before the configuration becomes unstable, i.e. between bursts during accretion. The regime of increased $\alpha$ in the data very nicely coincides with the regime of delayed mixed bursts in our models. Indeed, in this regime, much of the nuclear fuel does burn steadily, as suggested by van Paradijs et al. (1988), and it is only after a considerable surface density has accreted that the burst is initiated. Correspondingly, $\alpha$ goes up by a large factor. According to our models, in this regime, the critical $\Sigma_{\text {layer,crit }}$ to initiate a burst increases (Fig. 12), as does the recurrence time between bursts (Fig. 11), whereas the burst fluence as measured by $t_{\mathrm{H}+\mathrm{He}}$ is either unchanged or goes down (Fig. 13). All of these features are consistent with the observations, and this is one of the successes of the present work.

The sudden "break" from values of $\alpha \sim 100$ to larger values occurs at $\log \left(l_{\text {acc }}\right) \sim-1$ in the van Paradijs et al. (1988) data, though the precise location is hard to determine. A similar break has been seen in recent data presented by Cornelisse et al. (2003) who quote a break luminosity of $2.1 \times 10^{37} \mathrm{erg} \mathrm{s}^{-1}$ (they actually present data for burst rates and burst durations, but it is straightforward to translate these to $\alpha$ ). In our units, their break occurs at $\log \left(l_{\text {acc }}\right)=-0.9$, which is close to where we find a break in our models. This quantitative agreement is very encouraging. Cornelisse et al. (2003) interpret the break in terms of a transition from unstable to stable hydrogen burning, even though such a transition is expected to occur at very low luminosities, not at $\log \left(l_{\mathrm{acc}}\right) \sim-1$, and it does not predict the particular behavior seen in the data. In our model, the break signals the switch from the regime of prompt mixed bursts to that of delayed mixed bursts. Both the position of the break and the signatures we predict are in encouraging agreement with the observations.

At low accretion luminosities $\sim 0.01 L_{\text {Edd }}$, van Paradijs et al. (1988) find very low values of $\alpha \sim 10$. We are unable to reproduce this trend and are not aware of any other studies that succeed. Our models predict an increase in $\alpha$ as we move into the regime of helium bursts (where there is little or no hydrogen to burn), which is just the opposite of what is seen in the data. It would be useful to probe this regime in more detail with modern observations. 


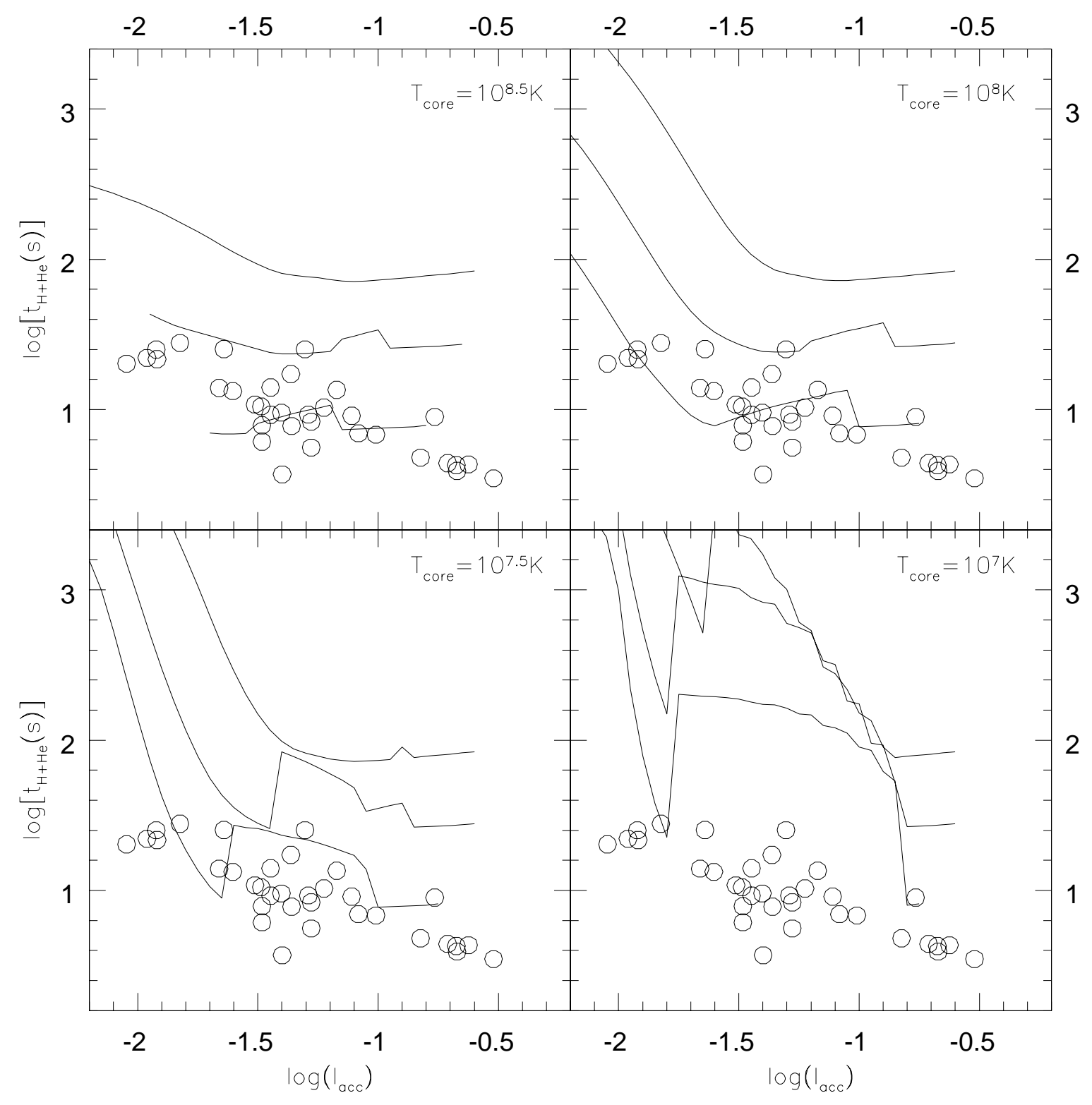

Fig. 19.- A comparison of the model burst durations $t_{\mathrm{H}+\mathrm{He}}$ with the corresponding data presented in van Paradijs et al. (1988). The model values are from Fig. 13, with the symbols replaced by connected lines. 


\subsubsection{Recurrence Time}

The results on $t_{\text {rec }}$ in Fig. 11 show a variety of patterns that could be tested against observations, but we are not aware of appropriate data in the literature. Continuous monitoring of sources, e.g., the recent work of Tournear et al. (2003) and Cornelisse et al. (2003), would be very useful in this regard. The latter paper finds that the peak burst rate in several sources is about 10 bursts per day, corresponding to a recurrence time of about 2.5 hours. (They also see sources with a peak burst rate of 2.5 per day, but these seem to belong to a separate class.) In our model, the shortest recurrence time is about 5 hours (Fig. 11), corresponding to a burst rate of about 5 bursts per day; this particular rate is found for smaller neutron star radii $(6.5 \mathrm{~km}$ and $10.4 \mathrm{~km})$ at the break point between the prompt mixed bursts and delayed mixed bursts at $\log \left(l_{\text {acc }}\right) \sim-1$. The location of the peak is in good agreement with the observations, but there is a factor of 2 discrepancy in the maximum burst rate. The latter may indicate that some of the approximations we have made (e.g., the opacity or the inner boundary condition) are still inadequate. It might also indicate that only a fraction of the fuel is burned during the burst.

The observations of Cornelisse et al. (2003) indicate in a few sources that the burst rate decreases proportional to the luminosity below the break point (e.g., GX 354-0 in their Fig. 2 ); equivalently, their recurrence times vary inversely as $t_{\mathrm{rec}} \propto l_{\mathrm{acc}}^{-1}$. Such a trend corresponds to a constant value of $\Sigma_{\text {layer,crit }}$, and arises naturally in our model (and in other models, e.g., see Figs. 16, 17). Among the models we have calculated, it appears that smaller stellar radii $(6.5 \mathrm{~km}, 10.4 \mathrm{~km})$ are more consistent with the observed trend than larger radii $(16.4 \mathrm{~km})$. Also, of the four core temperatures we have tried, we find best agreement with the data for the hotter temperatures $10^{7.5}, 10^{8}, 10^{8.5} \mathrm{~K}$. A core temperature of $10^{7} \mathrm{~K}$ appears unlikely.

Above the break, Cornelisse et al. (2003) find a decrease in the burst rate by a factor 4 . This is consistent with the increase in $t_{\text {rec }}$ seen in the models. Another interesting feature to check would be the sudden increase of $t_{\text {rec }}$ with decreasing $l_{\text {acc }}$ in the helium burst regime $\left(l_{\text {acc }}<-1.5\right)$. This regime has regions of very long recurrence times, which for all practical purposes may be considered burst-stable since observations are unlikely to detect these very rare bursts. We thus predict a "gap" in the occurrence of bursts for accretion luminosities in the vicinity of $\sim 10^{-2} L_{\mathrm{Edd}}$ (Narayan \& Heyl 2002). It is not that there are no bursts here, but that bursts are very rare and correspondingly very luminous (Fig. 13). It is not clear that the helium burst regime has been seen in the observations, or that there is a gap.

Indeed, the van Paradijs et al. (1988) give anomalously low values of $\alpha$ here, whereas helium bursts should have large values of $\alpha$. 


\subsubsection{Burst Fluence}

Figure 19 compares observed burst durations $\tau$ from the van Paradijs et al. (1988) compilation with our model predictions for the Eddington-scaled duration $t_{\mathrm{H}+\mathrm{He}}$. We see that the predicted durations are generally larger than the observed ones, though the models with a stellar radius of $6.5 \mathrm{~km}$ (the lowest lines in the panels) come pretty close. As in the case of the recurrence time, this comparison again is compromised by the possibility of partial burning of the fuel. Modulo this caveat, we conclude once again that somewhat

hotter cores $T_{\text {core }} \gtrsim 10^{7.5} \mathrm{~K}$ and smaller neutron star radii $R \lesssim 10 \mathrm{~km}$ are preferred.

One interesting feature in the models is that the burst duration drops by about a factor of $1.5-2$ above the break point at $\log \left(l_{\text {acc }}\right) \sim-1$. This is precisely the same point at which the burst recurrence time increases by a large factor. In the data presented by Cornelisse et al. (2003), such a decrease in the burst durations is seen (it is less obvious in the van Paradijs et al. data), but the amount of the decrease appears to be larger than we predict.

\subsubsection{Overstable Modes}

Revnivtsev et al. (2001) have reported a new class of low-frequency oscillations in burst sources that might be related to oscillatory behavior of the accreted layer prior to a burst. The oscillations seem to be present for accretion luminosities of about a tenth Eddington, and the frequency is about $0.01 \mathrm{~Hz}$.

Because our analysis computes the complex frequencies of unstable modes, it is natural to ask if we can explain the observed oscillations. The oscillations seem to be observed mostly in the regime that we have identified as delayed mixed bursts, above the break discussed in the previous subsections. In this regime, we do find complex values of $\gamma$ with the imaginary parts often larger than the real parts. However, our mode frequencies are generally lower than the observed frequencies by a factor of 10 or more. We do not know if this is an indication that some of our input physics is inadequate (\$6.1) or perhaps that our numerical technique for finding eigenmodes is unable to converge on modes with very high frequencies (high compared to the accretion frequency $\gamma_{\text {acc }}$ ). More work is needed. Alternatively, the oscillations may be related to slow propagation of the burning front on the stellar surface (see Bildsten 1995). 


\subsection{Future Prospects}

The richness of the properties of nuclear burning on neutron stars as revealed by the $a b$ initio linear stability analysis presented here is remarkable. Considering the encouraging agreement that we find with observational data, the model deserves further exploration. Since we have used a variety of prescriptions for calculating the opacity, the equation of state, the nuclear burning, etc., it might be useful to improve these aspects of the model. The radiative free-free opacity in the outer layers seems to have a large effect ( $\$ 6.1)$, so this is one area that needs more work. The treatment of the inner boundary condition is also a serious issue that needs to be considered in greater detail, especially for cool neutron star cores (see $\S 2.2)$.

One simplification in the present calculations is that we consider only a limited number of reactions for H-burning and He-burning (§2.3.3). At a sufficiently high temperature, breakout from the hot CNO cycle is expected to occur and hydrogen will be burned via the rapid proton capture (rp) process (Wallace \& Woosley 1982; Schatz et al. 1999). Figure 5 of Schatz et al. (1999) shows the temperature limits above which the reaction rates for processes such as ${ }^{15} \mathrm{O}(\alpha, \gamma){ }^{19} \mathrm{Ne}$ and ${ }^{14} \mathrm{O}(\alpha, p){ }^{17} \mathrm{~F}$ exceed the usual $\beta$-decay and proton capture rates for these nuclei. We have confirmed that all the burst-unstable configurations we have calculated in the present paper lie to the left of the corresponding lines in the Schatz et al. plot. Hence, none of our burst results are affected by the neglect of these additional reactions. However, close to the Eddington accretion rate, where our model predicts that the systems should be burst-stable, some of our equilibria cross into the zone where the breakout reactions, especially ${ }^{15} \mathrm{O}(\alpha, \gamma){ }^{19} \mathrm{Ne}$, become important. It is thus conceivable that some of the very high $\dot{M}$ configurations that we have identified as stable may turn out to be unstable when breakout reactions are included. This topic needs further investigation.

In terms of further calculations, the most obvious next step is to explore the effect

of varying the composition of the accreting gas. Variations in metallicity between burst sources in globular clusters and those in the Galactic disk may possibly have observational consequences. As a more extreme example, ultracompact binaries with degenerate helium secondaries should have very different properties from the hydrogen-dominant systems we have considered here. Obviously, without hydrogen, there will be no unstable hydrogen burning, so the hydrogen burst region will be missing from the results. Instead, we imagine the helium burst regime will extend both to lower and higher accretion rates than in the results presented in this paper. Because helium requires a higher temperature/density to ignite, the bursts should occur more rarely than for hydrogen-accreting neutron stars.

In Narayan \& Heyl (2002), we examined the burst properties of more massive primaries (black hole candidates) and found that the threshold for stable nuclear burning moves to a 
higher accretion rate. Hanawa \& Fujimoto (1982) argue that the properties of the nuclear burning to lowest order are functions of the surface gravity and the mass accretion rate; therefore, one can understand the mass dependence of burning regimes by keeping the mass fixed and varying the radius and accretion rate; specifically, increasing the mass of the primary shifts a diagram like Fig. 10 down and to the right which helps to account for the results of Narayan \& Heyl (2002). Even though this argument gives a qualitative understanding of the mass dependence of the burning regimes, serious quantitative applications requires the kind of detailed calculations we have presented here.

Recently, a class of very long X-ray bursts has been discovered (Cornelisse et al. 2000; Wijnands 2001) which have been interpreted as carbon-burning bursts (Taam \& Picklum 1978; Strohmayer 2000; Cumming \& Bildsten 2001; Strohmayer \& Brown 2002). In most cases, the bursts cannot be due to helium burning of a very thick layer since normal Type I bursts are seen at the same accretion rate, sometimes just before the superburst occurs. It would be interesting to include carbon-burning physics in our model to see what kinds of bursts are predicted. It is worth noting, however, that not all long bursts necessarily arise from carbon-burning. As we see in Fig. 13, we predict very long helium and even hydrogen bursts at low accretion luminosities under appropriate conditions. Some of the observed long bursts (e.g., Gotthelf \& Kulkarni 1997) may correspond to these.

On the observational front, there are numerous tests that one could envisage based on the results shown in Figs. 10-15. The different regimes of bursting — delayed mixed bursts, prompt mixed bursts, helium bursts, hydrogen bursts - reveal very distinct patterns in various observables. If these patterns are seen in the data, then one might be able to constrain the neutron star radius and/or the core temperature fairly well. This would have important implications for the neutron star equation of state and the nature of neutrino cooling in the core. We have made a beginning along these lines in this paper and have argued that perhaps $T_{\text {core }}$ is $\gtrsim 10^{7.5} \mathrm{~K}$ and neutron star radii are $\lesssim 10 \mathrm{~km}$. More work along these lines is worthwhile.

If we are to extend this model, which has been developed for neutron stars, to thermonuclear instabilities in accreting white dwarfs (classical novae), it would be necessary to include Coulomb corrections in the equation of state, a more accurate treatment of the semi-degenerate regime, and more detailed radiative and conductive opacities. Understanding whether there is a regime of stable nuclear burning at high accretion rates onto a white dwarf is a key ingredient of any scenario in which Type Ia supernovae result from stable accretion of mass onto a white dwarf (Hillebrandt \& Niemeyer 2000). Because classical novae typically eject the material accreted along with some of the substrate, a regime of stable nuclear burning is required for accretion to cause the white dwarf to grow in mass and to 
end up in a supernova. Unfortunately, our current prescriptions are too crude to treat this important burning regime accurately, but the potential of the technique for white dwarfs is tantalizing.

Finally, the methods that we have described here have deliberately avoided examination of the physics of the burst itself, during which many complications arise, including other nuclear reaction channels, convection and hydrodynamics. An important question to examine is whether our techniques could be extended in any simple way to study the properties of the bursts.

\section{Summary}

We have presented a comprehensive treatment of the stability of nuclear burning on the surface of neutron stars. For the first time, we have calculated the linearly unstable eigenmodes of an accretion layer in quasi-steady state, making no ad hoc assumptions regarding the criterion for instability. The model reproduces the various previously known regimes of nuclear burning on neutron stars, and agrees with earlier results where there is overlap. Additionally, we have been able to probe in detail the behavior of accreting neutron stars at high mass accretion rates, near the threshold of stable nuclear burning. Here, we find a hitherto unrecognized regime of delayed mixed bursts, with very distinct properties compared to the more standard prompt mixed bursts.

For accretion rates greater than one percent of the Eddington rate, we find encouraging agreement between the model predictions and observations of bursts. The existence of the regime of delayed mixed bursts provides a natural explanation for the observed dramatic increase of the burst parameter $\alpha$ at high accretion luminosities (Fig. 18 and Cornelisse et al. 2003). In addition, there is some indication from the preliminary comparisons presented here that burst systems have hot cores $\gtrsim 10^{7.5} \mathrm{~K}$, consistent with cooling in the neutron star interior being dominated by the modified URCA process or a similar low-efficiency cooling mechanism. Cool cores with $T_{\text {core }} \sim 10^{7} \mathrm{~K}$, as might be present if direct URCA cooling were to operate, are less likely. We also find a number of indications for small neutron star radii $\lesssim 10 \mathrm{~km}$. These results could be tightened with more careful modeling, e.g., by improving some of the prescriptions we use for the input physics ( 6.5$)$, and with more extensive and better quality data.

We thank Deepto Chakrabarty, Duncan Galloway, Erik Kuulkers, Feryal Ozel and Dimitrios Psaltis for useful discussions and comments. We are also grateful to the referee for a number of suggestions on the prescriptions used in $\S 2.3$ and for comments that helped to 
improve the presentation of the paper. R.N. was supported in part by NASA grant NAG510780. J.S.H. was supported by the National Aeronautics and Space Administration through Chandra Postdoctoral Fellowship Award Number PF0-10015 issued by the Chandra X-ray Observatory Center, which is operated by the Smithsonian Astrophysical Observatory for and on behalf of NASA under contract NAS8-39073.

\section{A. Equilibrium and Perturbations}

Here we discuss in more detail the basic equations describing the equilibrium and perturbations of the accreted layer. The governing equations are given in equations (3)-(7). We use the notation defined in Table 1 and in equations (14), (15), where quantities like $\rho_{0}, T_{0}$ refer to the equilibrium, $\rho, T$ refer to the corresponding quantities in the perturbed state, and $\rho_{1}, T_{1}$ refer to the spatial component of the linear perturbations. The equilibrium quantities have no time dependence, while the perturbations have a time dependence of the form $\exp (\gamma t)$. Since $\gamma$ is in general complex, the quantities $\rho, T, \rho_{1}, T_{1}$, etc. are complex, whereas $\rho_{0}, T_{0}$, etc. are real. Also, since $X_{0}+Y_{0}+Z_{0}=1$ and $X+Y+Z=1$, we have $X_{1}+Y_{1}+Z_{1}=0$.

Let us begin with the radiative transfer equation (4). For the equilibrium, this equation gives

$$
\frac{\partial T_{0}}{\partial \Sigma}=\frac{3 \kappa_{0} F_{0}}{16 \sigma T_{0}^{3}}
$$

where all quantities are real. For the perturbations, we linearize equation (4) and consider first-order deviations. This gives

$$
\frac{\partial T_{1}}{\partial \Sigma}=\frac{3 \kappa_{0}}{16 \sigma T_{0}^{3}} F_{1}+\frac{3 F_{0}}{16 \sigma}\left[\frac{\partial}{\partial \rho}\left(\frac{\kappa}{T^{3}}\right)_{0} \rho_{1}+\frac{\partial}{\partial T}\left(\frac{\kappa}{T^{3}}\right)_{0} T_{1}+\frac{\partial}{\partial X}\left(\frac{\kappa}{T^{3}}\right)_{0} X_{1}+\frac{\partial}{\partial Y}\left(\frac{\kappa}{T^{3}}\right)_{0} Y_{1}\right]
$$

where now the various quantities are in general complex. For instance, $\partial\left(\kappa / T^{3}\right) / \partial \rho$ refers to the derivative of the complex quantity $\kappa / T^{3}$ with respect to complex $\rho$ (the derivative is well-defined since all the quantities are analytic). In practice, we calculate such derivatives numerically. Since $\kappa$ in general depends on all three quantities $X, Y, Z$, it is necessary to replace $Z$ by $1-X-Y$ or $Z_{1}$ by $-X_{1}-Y_{1}$ before computing the partial derivatives $\partial / \partial X$, $\partial / \partial Y$.

Instead of writing an equation for the linear perturbation $T_{1}$, we could equally well consider the equation for the total (complex) perturbed temperature $T=T_{0}+T_{1} \exp (\gamma t) T_{1}$. 
This is nothing but the original equation

$$
\frac{\partial T}{\partial \Sigma}=\frac{3 \kappa F}{16 \sigma T^{3}}
$$

where $F$ is now the total complex perturbed flux, and $\kappa$ is the opacity corresponding to the perturbed $\rho, T, X, Y$. Within the linear approximation, this equation, coupled with equation (A1), has the same content as equation (A2) for the linear perturbations (equation A2 multiplied by $\exp (\gamma t)$ is just the difference of equations A3 and A1). Equation (A3) has the advantage of being more compact than equation (A2). It is also numerically more convenient, since the compactness of the equation translates to relative simplicity of the corresponding computer code.

Consider next the H-evolution equation (6), which involves a time derivative. The equilibrium is described by

$$
\dot{\Sigma} \frac{\partial X_{0}}{\partial \Sigma}=-\frac{\epsilon_{\mathrm{H}, 0}}{E_{\mathrm{H}}^{*}}
$$

where all quantities are real. The linear first order perturbation $X_{1}$ satisfies

$$
\gamma X_{1}+\dot{\Sigma} \frac{\partial X_{1}}{\partial \Sigma}=-\frac{1}{E_{\mathrm{H}}^{*}}\left[\left(\frac{\partial \epsilon_{H}}{\partial \rho}\right)_{0} \rho_{1}+\left(\frac{\partial \epsilon_{H}}{\partial T}\right)_{0} T_{1}+\left(\frac{\partial \epsilon_{H}}{\partial X}\right)_{0} X_{1}+\left(\frac{\partial \epsilon_{H}}{\partial Y}\right)_{0} Y_{1}\right]
$$

where the term $\gamma X_{1}$ on the left comes from the time derivative $\partial / \partial t$ operating on $\exp (\gamma t) X_{1}$. Once again, instead of considering the equation for the perturbation $X_{1}$, we may write down the equation for the total perturbed quantity $X=X_{0}+\exp (\gamma t) X_{1}$ :

$$
\gamma\left(X-X_{0}\right)+\dot{\Sigma} \frac{\partial X}{\partial \Sigma}=-\frac{\epsilon_{\mathrm{H}}}{E_{\mathrm{H}}^{*}}
$$

which is nearly identical in form to equation (A4) for the equilibrium, except that (i) it has an extra term proportional to $\gamma$ because of the time dependence of the perturbations, (i) $\epsilon_{\mathrm{H}}$ is evaluated at the perturbed $\rho, T, X, Y$, and (iii) all quantities are complex.

Following the above examples, the other three equations are straightforward. The hydrostatic equilibrium equation (3) gives for the equilibrium

$$
\frac{\partial P_{0}}{\partial \rho_{0}} \frac{\partial \rho_{0}}{\partial \Sigma}+\frac{\partial P_{0}}{\partial T_{0}} \frac{\partial T_{0}}{\partial \Sigma}+\frac{\partial P_{0}}{\partial X_{0}} \frac{\partial X_{0}}{\partial \Sigma}+\frac{\partial P_{0}}{\partial Y_{0}} \frac{\partial Y_{0}}{\partial \Sigma}=g
$$

and for the perturbations

$$
\frac{\partial P}{\partial \rho} \frac{\partial \rho}{\partial \Sigma}+\frac{\partial P}{\partial T} \frac{\partial T}{\partial \Sigma}+\frac{\partial P}{\partial X} \frac{\partial X}{\partial \Sigma}+\frac{\partial P}{\partial Y} \frac{\partial Y}{\partial \Sigma}=g
$$


The energy conservation equation gives

$$
\begin{aligned}
\frac{\partial F_{0}}{\partial \Sigma}= & -\dot{\Sigma} T_{0}\left[\left(\frac{\partial s}{\partial \rho}\right)_{0} \frac{\partial \rho_{0}}{\partial \Sigma}+\left(\frac{\partial s}{\partial T}\right)_{0} \frac{\partial T_{0}}{\partial \Sigma}+\left(\frac{\partial s}{\partial X}\right)_{0} \frac{\partial X_{0}}{\partial \Sigma}+\left(\frac{\partial s}{\partial Y}\right)_{0} \frac{\partial Y_{0}}{\partial \Sigma}\right]-\epsilon_{\mathrm{H}, 0}-\epsilon_{\mathrm{He}, 0}, \\
\frac{\partial F}{\partial \Sigma}= & -\gamma T\left[\left(\frac{\partial s}{\partial \rho}\right)\left(\rho-\rho_{0}\right)+\left(\frac{\partial s}{\partial T}\right)\left(T-T_{0}\right)+\left(\frac{\partial s}{\partial X}\right)\left(X-X_{0}\right)+\left(\frac{\partial s}{\partial Y}\right)\left(Y-Y_{0}\right)\right] \\
& -\dot{\Sigma} T\left[\left(\frac{\partial s}{\partial \rho}\right) \frac{\partial \rho}{\partial \Sigma}+\left(\frac{\partial s}{\partial T}\right) \frac{\partial T}{\partial \Sigma}+\left(\frac{\partial s}{\partial X}\right) \frac{\partial X}{\partial \Sigma}+\left(\frac{\partial s}{\partial Y}\right) \frac{\partial Y}{\partial \Sigma}\right]-\epsilon_{\mathrm{H}}-\epsilon_{\mathrm{He}},
\end{aligned}
$$

Finally, the He-evolution equation gives

$$
\begin{gathered}
\dot{\Sigma} \frac{\partial Y_{0}}{\partial \Sigma}=\frac{\epsilon_{\mathrm{H}, 0}}{E_{\mathrm{H}}^{*}}-\frac{\epsilon_{\mathrm{He}, 0}}{E_{\mathrm{He}}^{*}} \\
\gamma\left(Y-Y_{0}\right)+\dot{\Sigma} \frac{\partial Y}{\partial \Sigma}=\frac{\epsilon_{\mathrm{H}}}{E_{\mathrm{H}}^{*}}-\frac{\epsilon_{\mathrm{He}}}{E_{\mathrm{He}}^{*}} .
\end{gathered}
$$

Equations (A1), (A4), (A7), (A9) and (A11) are five differential equations for the equilibrium quantities $F_{0}, \rho_{0}, T_{0}, X_{0}, Y_{0}$. We assume a value for the outgoing flux $F_{\text {out }, 0}$ at the surface of the layer, solve for $\rho_{\text {out }, 0}, T_{\text {out }, 0}, X_{\text {out }, 0}, Y_{\text {out }, 0}$ from the outer boundary conditions, and then integrate the 5 differential equations down to the bottom of the layer and then into the substrate to a depth equal to $\Sigma_{\text {diff }}$ defined in equation (18). At this depth, we require the temperature $T_{0}\left(\Sigma_{\text {diff }}\right)$ to be equal to the required core temperature $T_{\text {core }}$. If it is not, we change the value of $F_{\text {out }, 0}$ and repeat until the inner boundary condition is satisfied. We then have a valid equilibrium solution.

For the perturbations, we work with equations (A3), (A6), (A8, (A10), (A12), which are five differential equations for the total perturbed quantities $F, \rho, T, X, Y$. At the surface, we set the flux equal to $F_{\text {out }}=F_{\text {out }, 0}+F_{\text {out }, 1}$, where $F_{\text {out }, 1} \ll F_{\text {out }, 0}$ in order to satisfy the assumption of a linear perturbation of the equilibrium. We solve for the other variables at the surface, assume a value for the eigenvalue $\gamma$, and integrate the equations down to a depth $\Sigma_{\max }$ in the substrate (see eq. 20). At this depth we require $T\left(\Sigma_{\max }\right)=T_{0}\left(\Sigma_{\max }\right)$. We vary $\gamma$ until this inner boundary condition is satisfied, at which point we have a solution for the complex eigenvalue $\gamma$, and also the shape of the eigenfunction $\left(F_{1}, \rho_{1}\right.$, etc.). The search in $\gamma$ space is tailored to find eigenvalues with positive real parts since only such modes are unstable.

Let us define a turning point in the sequence of equilibria (S-curve) to be a point at which the locus of equilibrium solutions satisfies the condition $d \Sigma_{\text {layer }} / d F_{\text {out }, 0}=0$. Since the derivative is zero, it means that two equilibria with escaping surface fluxes equal to $F_{\text {out }, 0}$ and 
$F_{\text {out }}=F_{\text {out }, 0}+F_{\text {out }, 1}$, where $F_{\text {out }, 1} \ll F_{\text {out }, 0}$, both satisfy the differential equations as well as the boundary conditions for the same value of $\Sigma_{\text {layer }}$. Now, the solution for $F_{\text {out }, 0}$ satisfies the equilibrium equations (A1), (A4), (A7), (A9), (A11), while the solution for $F_{\text {out }}$ satisfies the perturbed equations (A3), (A6), (A8), (A10), (A12). The only difference between the two sets of equations is the presence of various terms involving $\gamma$. If the second solution is also an equilibrium solution, then it implies that it must satisfy the perturbation equations with $\gamma$ precisely equal to 0 , i.e., one of the modes of the system corresponds to 0 frequency. This result is not surprising. Since the initial model is at a turning point, there are neighboring equilibria with the same value of $\Sigma_{\text {layer }}$ but different values of $F_{\text {out, } 0}$, i.e., the system has a linear zero-frequency mode.

The above theorem, that a mode with $\gamma=0$ exists at turning points of the S-curve, is well-known for one-zone models, e.g., see the discussion of Paczynski (1983a) for an application to bursts. Our discussion shows that the same result is valid even when one is considering the more complex model described here. The reason it works is that our equilibria are ultimately labeled by a single parameter, the value of $F_{\text {out }, 0}$ at the surface. This is all that matters, and the fact that our solutions involve many variables and are described by continuous functions is not relevant.

\section{REFERENCES}

Allen, C. W. 2000, Allen's Astrophysical Quantities, fourth edition, ed. A. N. Cox, AIP Press (New York: Springer-Verlag)

Antia, H. M. 1993, ApJS, 84, 101

Bildsten, L. 1995, ApJ, 438, 852

Bildsten, L. 1998, in The Many Faces of Neutron Stars, ed. A. Alpar, L. Buccheri, \& J. van Paradij (Dordrecht: Kluwer), 419, astro-ph/9709094

Bildsten, L. 2000, in Rossi2000: Astrophysics with the Rossi X-ray Timing Explorer. March 22-24, 2000 at NASA's Goddard Space Flight Center, Greenbelt, MD USA, p.E65

Bildsten, L. \& Cumming, A. 1998, ApJ, 506, 842

Brown, E. F. 2000, ApJ, 531, 988

Brown, E. F. \& Bildsten, L. 1998, ApJ, 496, 915

Brown, E. F., Bildsten, L., \& Rutledge, R. E. 1998, ApJ, 504, L95 
Clayton, D. D. 1983, Principle of Stellar Evolution and Nucleosynthesis (Chicago: The University of Chicago Press)

Cornelisse, R., Heise, J., Kuulkers, E., Verbunt, F., \& in’t Zand, J. J. M. 2000, å, 357, L21

Cornelisse, R., in't Zand, J. J. M., Verbunt, F., Kuulkers, E., Heise, J., et al. 2003, å, in press (astro-ph/0304500)

Cumming, A. \& Bildsten, L. 2000, ApJ, 544, 453

Dewitt, H. E., Graboske, H. C., \& Cooper, M. S. 1973, ApJ, 181, 439

Epstein, R. I., Gudmundsson, E. H., \& Pethick, C. J. 1983, MNRAS, 204, 471

Ergma, E. V. \& Tutukov, A. V. 1980, A\&A, 84, 123

Fujimoto, M. Y., Hanawa, T., Iben, I. J., \& Richardson, M. B. 1987a, ApJ, 315, 198

Fujimoto, M. Y., Hanawa, T., \& Miyaji, S. 1981, ApJ, 247, 267

Fujimoto, M. Y., Sztajno, M., Lewin, W. H. G., \& van Paradijs, J. 1987b, ApJ, 319, 902

Fushiki, I. \& Lamb, D. Q. 1987a, ApJ, 323, L55

-. 1987b, ApJ, 317, 368

Gotthelf, E. V., \& Kulkarni, S. R. 1997, ApJ, 490, L161

Grindlay, J., Gursky, H., Schnopper, H., Parsignault, D. R., Heise, J., Brinkman, A. C., \& Schrijver, J. 1976, ApJ, 205, L127

Hanawa, T. \& Fujimoto, M. Y. 1982, PASJ, 34, 495

—. 1986, PASJ, 38, 13

Hansen, C. J. \& van Horn, H. M. 1975, ApJ, 195, 735

Hernquist, L. \& Applegate, J. H. 1984, ApJ, 287, 244

Hillebrandt, W. \& Niemeyer, J. C. 2000, ARA\&A, 38, 191

Iben, I. 1975, ApJ, 196, 525

Itoh, N., Tomizawa, N., Wanajo, S., \& Nozawa, S. 2003, ApJ, 586, 1436

Joss, P. C. 1977, Nature, 270, 310 
—. 1978, ApJ, 225, L123

Joss, P. C. \& Li, F. K. 1980, ApJ, 238, 287

Kuulkers, E., \& van der Klis, M. 2000, å, 356, L45

Kuulkers, E., van der Klis, M., \& van Paradijs, J. 1995, ApJ, 450, 748

Lewin, W. H. G., van Paradijs, J., \& Taam, R. E. 1993, Space Science Reviews, 62, 223+

Matsuba, E., Dotani, T., Misuda, K., Asai, K., Lewin, W. H. G., van Parakijs, J., \& van der Klis, M. 1995, PASJ, 47, 575

Mathews, G. J. \& Dietrich, F. S. 1984, ApJ, 287, 969

Müller, D. E. 1956, Mathematical Tables and Aids to Computation, 10, 208

Muno, M. P., Chakrabarty, D., Galloway, D. K., \& Savov, P. 2001, ApJ, 553, L157

Narayan, R. \& Heyl, J. S. 2002, ApJ, 574, 139

Paczynski, B. 1983a, ApJ, 264, 282

—. 1983b, ApJ, 267, 315

Potekhin, A. Y. 1999, A\&A, 351, 787

Regev, O. \& Livio, M. 1984, A\&A, 134, 123

Revnivtsev, M., Churazov, E., Gilfanov, M., \& Sunyaev, R. 2001, A\&A, 372, 138

Schatz, H., Bildsten, L., Cumming, A., \& Wiescher, M. 1999, ApJ, 524, 1014

Shapiro, S. L. \& Teukolsky, S. A. 1983, Black Holes, White Dwarfs, and Neutron Stars (New York: Wiley-Interscience)

Smale, A. P. 1998, ApJ, 498, L141

Strohmayer, T., Zhang, W., Swank, J. H., Smale, A., Titarchuk, L., Day, C., \& Lee, U. 1996, ApJ, 469, L9

Strohmayer, T. 2001, Adv. Space Res., 28, 511

Strohmayer, T. 2000, AAS HEAD Meeting, 32, 24.10

Strohmayer, T., \& Brown, E. F. 2002, ApJ, 566, 1045 
Strohmayer, T. E., Swank, J. H., \& Zhang, W. 1998, Nucl. Phys. B (Proc. Suppl.), 69, 129, astro-ph/9801219

Taam, R. E. 1982, ApJ, 258, 761

Taam, R. E. \& Picklum, R. E. 1978, ApJ, 224, 210

-. 1979, ApJ, 233, 327

Taam, R. E., Woosley, S. E., \& Lamb, D. Q. 1996, ApJ, 459, 271

Taam, R. E., Woosley, S. E., Weaver, T. A., \& Lamb, D. Q. 1993, ApJ, 413, 324

Tournear, D., et al. 2003, preprint

van Paradijs, J., Penninx, W., \& Lewin, W. H. G. 1988, MNRAS, 233, 437

van Straaten, S., van der Klis, M., Kuulkers, E., \& Mendez, M. 2001, ApJ, 551, 907

Wijnands, R. 2001, ApJ, 554, L59

Wallace, R. K., \& Woosley, S. E. 1982, ApJ, 258, 696

Woosley, S. E. \& Taam, R. E. 1976, Nature, 263, 101

Zingale, M., Timmes, F. X., Fryxell, B., Lamb, D. Q., Olson, K., et al. 2001, ApJS, 133, 195 